Supporting information for

\title{
Enantioselective Iridium-Catalyzed Allylic Alkylation of Racemic Branched Alkyl-Substituted Allylic Acetates with \\ Malonates
}

\author{
Tian-Yuan Zhang, ${ }^{a, b, \dot{\ddagger}}$ Yi Deng, ${ }^{a, b, \grave{\ddagger}}$ Kun Wei, ${ }^{a}$ and Yu-Rong Yang ${ }^{*}, a$ \\ ${ }^{a}$ State Key Laboratory of Phytochemistry and Plant Resources in West China, Kunming \\ Institute of Botany, Chinese Academy of Sciences, Kunming 650201, China \\ ${ }^{b}$ University of Chinese Academy of Sciences, Beijing 100049, China \\ yangyurong@mail.kib.ac.cn
}

\section{Content}

General Data

Optimization of reaction conditions

1. Solvent screening

2. Temperature screening

3. Ligand screening

4. Base and temperature screening

5. Leaving group screening

6. Compatibility of aryl-substituted substrates

Synthesis and characterization of substrates and products

References

NMR Data

HPLC Data 


\section{General Data}

Unless otherwise stated, all oxygen or moisture sensitive reactions were conducted in flame-dried glassware under an atmosphere of nitrogen. All solvents were purified and dried according to standard methods prior to use. The corresponding ligands $(R)$-Ir-Ligand, allylic acetate and dibenzyl malonate were prepared according to the reported procedure ${ }^{[1][2][3]}$. Dimethyl malonate and other reagents were purchased from commercial sources and were used without further purification.

Chromatographic purification of products was accomplished using forced-flow chromatography on 200-300 mesh silica gel. The TLC glass plates were performed on $0.20 \mathrm{~mm}$ or $1.0 \mathrm{~mm}$ (preparative) silica gel GF254 plates. Visualization was performed using ultraviolet light $(254 \mathrm{~nm})$, potassium permanganate $\left(\mathrm{KMnO}_{4}\right)$ in water.

${ }^{1} \mathrm{H}$ and ${ }^{13} \mathrm{C}$ NMR spectra were acquired on Bruker Avance III-400 spectrometer and Bruker Avance III-600MHz spectrometer (Bruker Corp., Germany), TMS was used as internal standard. Chemical shifts were given in parts per million (ppm) with reference to residual solvent signals $\left[{ }^{1} \mathrm{H}\right.$ NMR: $\mathrm{CDCl}_{3}$ (7.26); ${ }^{13} \mathrm{C}$ NMR: $\mathrm{CDCl}_{3}$ (77.0)]. Peak multiplicities were recorded as follows: $\mathrm{s}=$ singlet, $\mathrm{d}=$ doublet, $\mathrm{t}=$ triplet, $\mathrm{q}=$ quartet, $\mathrm{m}=$ multiplet or unresolved, br = broad singlet. Infrared (IR) spectra were recorded on a Nicolet iS10 Fourier transform infrared (FT-IR) spectrometer (Thermo Scientific, United States) with $\mathrm{KBr}$ pellets. High resolution mass spectral (HRMS) data were obtained at the mass spectrometry service operated at a Shimadzu UPLC-IT-TOF spectrometer (Shimadzu Corp., Japan) for electrospray ionization (ESI) were reported as $(\mathrm{m} / \mathrm{z})$. Optical rotations were measured on a Jasco P-1020 Polarimeter (Jasco Corp., Japan). HPLC analysis was performed on an Agilent 1260 series system using Daicel chiralpak AD-H and IC with $n$-hexane and $i-\mathrm{PrOH}$ as solvents. 


\section{Optimization of reaction conditions}<smiles>[R]OC(C=C)CCc1ccccc1</smiles>

$10 \mathrm{mmol} \%$<smiles>COCCC(C)OC</smiles>

$20 \mathrm{mmol} \%$ $\underset{\text { Base }(20 \mathrm{mmol} \%)}{\stackrel{(R) \text {-Ir-Ligand }(0.5 \mathrm{mmol} \%)}{\longrightarrow}}$ solvent $(0.5 \mathrm{M})$ $\mathrm{T}^{\circ} \mathrm{C}$

$\mathrm{R}=\mathrm{Ac}, \mathrm{Boc}, \mathrm{PO}(\mathrm{OEt})_{2}, \mathrm{Bz}$

General procedure: A pressure tube equipped with a magnetic stir bar was charged with cesium carbonate $(20 \mathrm{mmol} \%),(R)$-Ir-Ligand $(0.5 \mathrm{mmol} \%)$, corresponding allylic substrates $(10 \mathrm{mmol} \%)$ and the dimethyl malonate $(20 \mathrm{mmol} \%)$, DME $(0.5 \mathrm{M})$ was added. The tube was purged with argon for 1 minute. The tube was sealed with a PTFE lined cap and was placed in an oil bath at the indicated temperature and stirred for the indicated period of time. After reaching ambient temperature, the crude reaction mixture was directly subjected to flash column chromatography.

\section{Solvent screening}

Standard conditions: Following general procedure, allylic acetate $(10 \mathrm{mmol} \%)$, dimethyl malonate (20 mmol\%), (R)-Ir-SEGPHOS (0.5 mmol\%), $\mathrm{Cs}_{2} \mathrm{CO}_{3}(20 \mathrm{mmol} \%)$, solvent $(0.5 \mathrm{M}), 70{ }^{\circ} \mathrm{C}, 20 \mathrm{~h}$.

\begin{tabular}{cccc}
\hline entry & solvent & yield $(\%)^{\mathrm{a}}$ & $\operatorname{er}^{\mathrm{b}}$ \\
\hline $1^{\mathrm{c}}$ & DME & 83 & $85: 15$ \\
2 & THF & 33 & $76: 24$ \\
$3^{\mathrm{d}}$ & Dioxane & 78 & $74: 26$ \\
\hline
\end{tabular}

${ }^{a}$ Isolated yield. ${ }^{b}$ The er value was determined by HPLC on an IC column. ${ }^{\mathrm{c}, \mathrm{d}}$ The reaction concentration was $1.0 \mathrm{M}$

\section{Temperature screening}

Standard conditions: Following general procedure, allylic acetate $(10 \mathrm{mmol} \%)$, dimethyl malonate (20 mmol\%), (R)-Ir-SEGPHOS (0.5 mmol\%), $\mathrm{Cs}_{2} \mathrm{CO}_{3}(20 \mathrm{mmol} \%)$, DME (0.5 M), $40 \mathrm{~h}$.

\begin{tabular}{cccc}
\hline entry & $\mathrm{T}\left({ }^{\circ} \mathrm{C}\right)$ & $\operatorname{yield}(\%)^{\mathrm{a}}$ & $\operatorname{er}^{\mathrm{b}}$ \\
\hline 1 & 70 & 83 & $85: 15$ \\
2 & 50 & 85 & $88: 12$
\end{tabular}


${ }^{a}$ Isolated yield. ${ }^{b}$ The er value was determined by HPLC on an IC column.

\section{Ligand screening}

Standard conditions: Following general procedure, allylic acetate (10 $\mathrm{mmol} \%)$, dimethyl malonate (20 mmol\%), (R)-Ir-Ligand (0.5 mmol\%), $\mathrm{Cs}_{2} \mathrm{CO}_{3}(20 \mathrm{mmol} \%), \mathrm{DME}(0.5 \mathrm{M}), 50{ }^{\circ} \mathrm{C}, 40 \mathrm{~h}$.

\begin{tabular}{cccc}
\hline entry & ligand & yield $(\%)^{\mathrm{a}}$ & $\mathrm{er}^{\mathrm{b}}$ \\
\hline 1 & $(R)$-Ir-SEGPHOS & 85 & $88: 12$ \\
$2^{\mathrm{c}}$ & $(R)$-Ir-SDP & 58 & $54: 46$ \\
3 & $(R)$-Ir-Tol-BINAP & 94 & $97: 3$ \\
$4^{\mathrm{d}}$ & $(R)$-Ir-H -BINAP & 36 & $88: 12$ \\
5 & $(R)$-Ir-DM-BINAP & 94 & $89: 11$ \\
\hline
\end{tabular}

${ }^{a}$ Isolated yield. ${ }^{\mathrm{b}}$ The er value was determined by HPLC on an IC column. ${ }^{\text {cd. }}$ Run in $5 \mathrm{mmol} \%$ allylic acetate.

\section{Base and temperature screening}

Standard conditions: Following general procedure, allylic acetate (10 $\mathrm{mmol} \%)$, dimethyl malonate (20 mmol\%), (R)-Ir-Ligand (0.5 mmol\%), base (20 mmol\%), DME (0.5 M) ,40 h.

\begin{tabular}{cccccc}
\hline entry & base & $\mathrm{T}\left({ }^{\circ} \mathrm{C}\right)$ & ligand & yield $(\%)^{\mathrm{a}}$ & $\mathrm{er}^{\mathrm{b}}$ \\
\hline 1 & $\mathrm{Cs}_{2} \mathrm{CO}_{3}$ & 70 & $(R)$-Ir-Tol-BINAP & 88 & $95: 5$ \\
2 & $\mathrm{Cs}_{2} \mathrm{CO}_{3}$ & 50 & $(R)$-Ir- Tol -BINAP & 94 & $97: 3$ \\
3 & $\mathrm{Cs}_{2} \mathrm{CO}_{3}$ & 70 & $(R)$-Ir-SEGPHOS & 83 & $85: 15$ \\
4 & $\mathrm{~K}_{2} \mathrm{CO}_{3}$ & 70 & $(R)$-Ir- Tol -BINAP & 72 & $94: 6$ \\
5 & $\mathrm{~K}_{2} \mathrm{CO}_{3}$ & 50 & $(R)$-Ir- Tol -BINAP & 32 & $94: 6$ \\
5 & $\mathrm{~K}_{2} \mathrm{CO}_{3}$ & 70 & $(R)$-Ir-SEGPHOS & 69 & $80: 20$ \\
6 & $\mathrm{~K}_{3} \mathrm{PO}_{4}$ & 70 & $(R)$-Ir- Tol -BINAP & 62 & $97: 3$ \\
7 & $\mathrm{~K}_{3} \mathrm{PO}_{4}$ & 50 & $(R)$-Ir- Tol -BINAP & 39 & $97: 3$ \\
8 & $\mathrm{~K}_{3} \mathrm{PO}_{4}$ & 70 & $(R)$-Ir-SEGPHOS & 45 & $84: 16$ \\
9 & $t-\mathrm{BuOLi}$ & 70 & $(R)$-Ir- Tol -BINAP & 87 & $89: 11$ \\
10 & $t$-BuOLi & 50 & $(R)$-Ir- Tol -BINAP & 65 & $92: 8$ \\
11 & $t$-BuONa & 70 & $(R)$-Ir- Tol -BINAP & 76 & $91: 9$ \\
12 & $\mathrm{KOAc}$ & 70 & $(R)$-Ir- Tol -BINAP & - & - \\
13 & $\mathrm{NaOAc}$ & 70 & $(R)$-Ir- Tol -BINAP & - & - \\
\hline
\end{tabular}

${ }^{a}$ Isolated yield. ${ }^{\mathrm{b}}$ The er value was determined by HPLC on an IC column 


\section{Leaving group screening}

Standard conditions: Following general procedure, corresponding allylic substrates (10 mmol\%), dimethyl malonate (20 mmol\%), (R)-Ir-Tol-Ligand (0.5 mmol\%), $\mathrm{Cs}_{2} \mathrm{CO}_{3}(20 \mathrm{mmol} \%)$, DME $(0.5$ M), $50{ }^{\circ} \mathrm{C}, 40 \mathrm{~h}$.

\begin{tabular}{cccc}
\hline entry & leaving group & yield $(\%)^{\mathrm{a}}$ & $\mathrm{er}^{\mathrm{b}}$ \\
\hline 1 & $-\mathrm{OAc}$ & 94 & $97: 3$ \\
2 & $-\mathrm{OBoc}$ & 91 & $91: 9$ \\
3 & $-\mathrm{OPO}(\mathrm{OEt})_{2}$ & 89 & $95: 5$ \\
4 & $-\mathrm{OBz}$ & 20 & $96: 4$ \\
\hline
\end{tabular}

a Isolated yield. ${ }^{\mathrm{b}}$ The er value was determined by HPLC on an IC column

\section{Compatibility of aryl-substituted substrate}

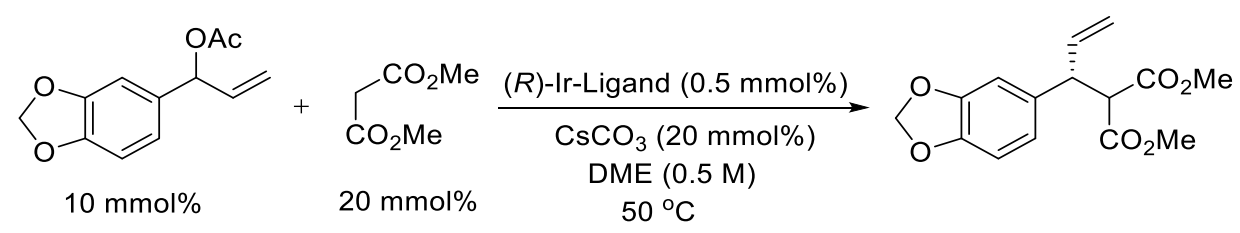

Standard conditions: Following general procedure, piperonyl allylic acetate (10 mmol\%), dimethyl malonate (20 mmol\%), (R)-Ir -Ligand (0.5 mmol\%), $\mathrm{Cs}_{2} \mathrm{CO}_{3}(20 \mathrm{mmol} \%)$, DME (0.5 M), $50{ }^{\circ} \mathrm{C}, 40 \mathrm{~h}$.

\begin{tabular}{cccc}
\hline entry & catalyst & yield $(\%)^{\mathrm{a}}$ & $\mathrm{er}^{\mathrm{b}}$ \\
\hline 1 & $(R)-$ Ir-Tol-BINAP & 92 & $52: 48$ \\
$2^{\mathrm{c}}$ & $(R)-$ Ir-SDP & 92 & $69: 31$ \\
3 & $(R)-$ Ir-SEGPHOS & 90 & $89: 11$ \\
$4^{\mathrm{d}}$ & $(R)-\mathrm{Ir}-\mathrm{H}_{8}$-BINAP & 96 & $65: 35$ \\
\hline
\end{tabular}

${ }^{a}$ Isolated yield. ${ }^{\mathrm{b}}$ The er value was determined by HPLC on an AD-H column. ${ }^{\text {cd. }}$ Run in 5 mmol\% piperonyl allylic acetate. 


\section{Synthesis and characterization of substrates and products}

\section{General procedure for preparation of allylic acetates ${ }^{[1]}$}

\section{General procedure :}

Vinyl magnesium bromide (1.0 $\mathrm{M}$ in THF, $3.6 \mathrm{mmol}$ ) was added dropwise to a solution of the corresponding aldehyde $(3.0 \mathrm{mmol})$ in dry $\mathrm{THF}$ at $0^{\circ} \mathrm{C}$, and the mixture was stirred at room temperature for 10 minutes, at which point triethylamine $(6.0 \mathrm{mmol})$ and acetic anhydride $(4.5 \mathrm{mmol})$ were added and the reaction was stirred vigorously for 30 minutes. After water was added, the organic layer was extracted with diethyl ether and the combined organic layers were washed with 1 $\mathrm{N} \mathrm{HCl}$, brine, dried over $\mathrm{Na}_{2} \mathrm{SO}_{4}$, concentrated in vacuo and then purified by silica gel column chromatography to afford the corresponding allylic acetates.

\section{Characterization of allylic acetates:}

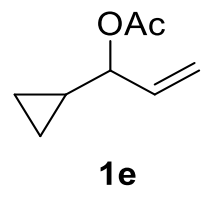

The compound was prepared according to the general procedure. The crude reaction mixture was purified on silica gel (petroleum ether/ethyl acetate 15/1) to afford 1e as a colorless oil (180 mg, 43\%).

$\mathbf{R}_{f}=0.43$ (petroleum ether/ethyl acetate 10/1). ${ }^{1} \mathbf{H}$ NMR (400 MHz, $\left.\mathrm{CDCl}_{3}\right) \delta 5.85$ (ddd, J = 6.0, 10.5, 16.8 Hz, 1H), 5.27 (d, J = 16.8 Hz, 1H), $5.16(\mathrm{~d}, \mathrm{~J}=10.5 \mathrm{~Hz}, 1 \mathrm{H}), 4.71$ (dd, J = 6.0, $6.3 \mathrm{~Hz} 1 \mathrm{H}), 2.08(\mathrm{~s}, 3 \mathrm{H}), 1.10(\mathrm{~m}, 1 \mathrm{H}), 0.51-0.51(\mathrm{~m}, 2 \mathrm{H}), 0.42(\mathrm{~m}, 1 \mathrm{H}), 0.31(\mathrm{~m}, 1 \mathrm{H}) .{ }^{13} \mathrm{C}$ NMR (100 MHz, $\left.\mathrm{CDCl}_{3}\right) \delta 167.9,133.1,114.0,76.1,18.8,12.1,1.0,0.0$ ppm. FT-IR (KBr): 2958, 2926, 2856, 1739, 1431, 1369, 1240, 1099, 1025, 967, $804 \mathrm{~cm}^{-1}$. HRMS $\left(\mathrm{ESI}^{+}\right): \mathrm{m} / \mathrm{z}$ calcd for $\mathrm{C}_{8} \mathrm{H}_{13} \mathrm{O}_{2}[\mathrm{M}+\mathrm{H}]^{+}$141.0910, found 141.0908 .

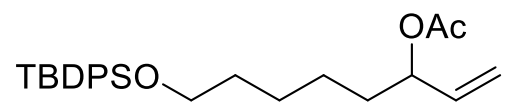

$1 \mathbf{j}$

The compound was prepared according to the general procedure. The crude reaction mixture was purified on silica gel (petroleum ether/ethyl acetate 20/1) to afford $\mathbf{1} \mathbf{j}$ as a colorless oil $(1.1 \mathrm{~g}, 88 \%)$. 
$\mathbf{R}_{f}=0.62$ (petroleum ether/ethyl acetate 10/1). ${ }^{1} \mathbf{H}$ NMR $\left(400 \mathrm{MHz}, \mathrm{CDCl}_{3}\right) \delta 7.70-7.67(\mathrm{~m}$, 4H), 7.43-7.37 (m, 6H), 5.78 (ddd, J = 6.4, 10.5, 17.2 Hz, 1H), $5.26-5.22(\mathrm{~m}, 2 \mathrm{H}), 5.17$ (d, J $=10.5 \mathrm{~Hz}, 1 \mathrm{H}), 3.67(\mathrm{t}, \mathrm{J}=6.4,2 \mathrm{H}), 2.07(\mathrm{~s}, 3 \mathrm{H}), 1.66-1.56(\mathrm{~m}, 4 \mathrm{H}), 1.43-1.27(\mathrm{~m}, 4 \mathrm{H}), 1.09$ (s, 9H). ${ }^{13} \mathbf{C}$ NMR $\left(100 \mathrm{MHz}, \mathrm{CDCl}_{3}\right) \delta 170.4,136.6,135.6,134.1,129.6,127.6,116.6,74.9$, 63.8, 34.2, 32.4, 26.9, 25.7, 24.9, 21.3, 19.3.ppm. FT-IR (KBr): 3071, 3049, 3015, 2932, 2897, 2858, 1739, 1589, 1473, 1462, 1389, 1370, 1239, 1111, 1020, 932, 740, $703 \mathrm{~cm}^{-1}$. HRMS $\left(\mathrm{ESI}^{+}\right): \mathrm{m} / z$ calcd for $\mathrm{C}_{26} \mathrm{H}_{36} \mathrm{O}_{3} \mathrm{SiNa}[\mathrm{M}+\mathrm{Na}]^{+}$447.2326, found 447.2333.

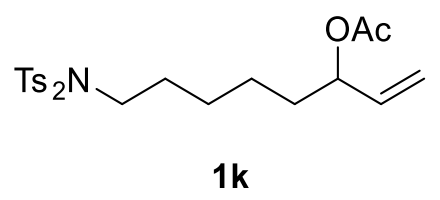

The compound was prepared according to the general procedure. The crude reaction mixture was purified on silica gel (petroleum ether/ethyl acetate 10/1) to afford $\mathbf{1 k}$ as a colorless oil (930 mg, 63\% yield).

$\mathbf{R}_{f}=0.67$ (petroleum ether/ethyl acetate 5/1). ${ }^{1} \mathbf{H} \mathbf{~ N M R}\left(400 \mathrm{MHz}, \mathrm{CDCl}_{3}\right) \delta 7.88(\mathrm{~d}, \mathrm{~J}=8.2$ $\mathrm{Hz}, 4 \mathrm{H}), 7.32(\mathrm{~d}, \mathrm{~J}=8.2 \mathrm{~Hz}, 4 \mathrm{H}), 5.73(\mathrm{ddd}, \mathrm{J}=6.6,10.3,17.7 \mathrm{~Hz}, 1 \mathrm{H}), 5.23-5.14(\mathrm{~m}, 3 \mathrm{H})$, $3.62(\mathrm{t}, \mathrm{J}=6.1,2 \mathrm{H}), 2.44(\mathrm{~s}, 6 \mathrm{H}), 2.05(\mathrm{~s}, 3 \mathrm{H}), 1.70-1.65(\mathrm{~m}, 2 \mathrm{H}), 1.56-1.50(\mathrm{~m}, 2 \mathrm{H}), 1.40-$ $1.02(\mathrm{~m}, 4 \mathrm{H}) .{ }^{13} \mathbf{C}$ NMR $\left(100 \mathrm{MHz}, \mathrm{CDCl}_{3}\right) \delta 170.4,144.8,137.2,136.5,129.7,128.2,116.7$, 74.6, 49.2, 34.0, 29.7, 26.3, 24.5, 21.7, 21.3 ppm. FT-IR (KBr): 3070, 3031, 2992, 2943, 2863 , 1735, 1597, 1493, 1454, 1371, 1353, 1242, 1187, 1165, 1085, 1019, 848, 814, $703 \mathrm{~cm}^{-1}$. HRMS $\left(\mathrm{ESI}^{+}\right): \mathrm{m} / z$ calcd for $\mathrm{C}_{24} \mathrm{H}_{31} \mathrm{NO}_{6} \mathrm{~S}_{2} \mathrm{Na}[\mathrm{M}+\mathrm{Na}]^{+}$516.1485, found 516.1485.

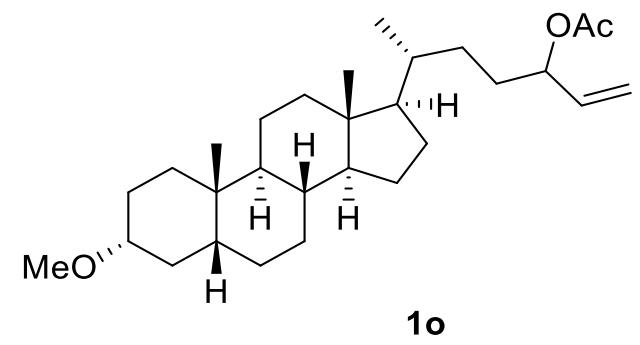

The compound was prepared according to the general procedure. The crude reaction mixture was purified on silica gel (petroleum ether/ethyl acetate 20/1) to afford 10 as a colorless oil (220mg, 50\% yield).

$\mathbf{R}_{f}=0.62$ (petroleum ether/ethyl acetate 10/1). ${ }^{1} \mathbf{H}$ NMR (400 MHz, $\left.\mathrm{CDCl}_{3}\right) \delta 5.76$ (ddd, J = 6.5, 10.7, $14.9 \mathrm{~Hz}, 1 \mathrm{H}), 5.25(\mathrm{~m}, 1 \mathrm{H}), 5.21-5.14(\mathrm{~m}, 2 \mathrm{H}), 3.35$ (s, 3H), 3.16 (dt, J = 5.7, 10.9 
Hz, 1H), $2.06(\mathrm{~s}, 3 \mathrm{H}), 1.94(\mathrm{~d}, \mathrm{~J}=12.2 \mathrm{~Hz}, 1 \mathrm{H}), 1.88-1.72(\mathrm{~m}, 4 \mathrm{H}), 1.68-1.53(\mathrm{~m}, 6 \mathrm{H}), 1.48$ - $1.34(\mathrm{~m}, 7 \mathrm{H}), 1.30-1.16(\mathrm{~m}, 4 \mathrm{H}), 1.17-0.99(\mathrm{~m}, 6 \mathrm{H}), 0.90-0.89(\mathrm{~m}, 6 \mathrm{H}), 0.63(\mathrm{~s}, 3 \mathrm{H}) .{ }^{13} \mathrm{C}$ NMR $\left(150 \mathrm{MHz}, \mathrm{CDCl}_{3}\right) \delta 170.5,170.4,136.8,136.6,116.8,116.4,80.4,75.5,75.1,56.5$, 55.9, 55.9, 55.6, 42.7, 42.0, 40.3, 40.2, 35.8, 35.4, 35.4, 35.3, 34.9, 32.8, 31.0, 31.0, 30.7, 30.6, 29.7, 28.2, 28.2, 27.3, 26.8, 26.4, 24.2, 23.4, 21.3, 20.8, 18.6, 18.5, 12.0 ppm. FT-IR (KBr): 2934, 2865, 2820, 1740, 1466, 1448, 1371, 1238, 1174, 1100, 1019, 986, $928 \mathrm{~cm}^{-1}$. HRMS $\left(\mathrm{ESI}^{+}\right): \mathrm{m} / z$ calcd for $\mathrm{C}_{29} \mathrm{H}_{48} \mathrm{O}_{3} \mathrm{Na}[\mathrm{M}+\mathrm{Na}]^{+} 467.3496$, found 467.3499 .

\section{General procedure for preparation of dibenzyl malonate ${ }^{[2]}$}

In a round bottom flask equipped with a Dean-Stark trap, $3.00 \mathrm{~g}(28.8 \mathrm{mmol})$ malonic acid, $9.78 \mathrm{~g}$ (90.4 mmol) benzyl alcohol and $0.35 \mathrm{~g}(2.0 \mathrm{mmol}) \mathrm{p}$-Toluenesulfonic acid were added to $30 \mathrm{~mL}$ toluene. The reaction mixture was heated under reflux for $20 \mathrm{~h}$. After cooling to room temperature, $10 \mathrm{~mL}$ water was added to the mixture and the layers were separated. The organic phase was washed with sat. $\mathrm{NaHCO}_{3}$ solution $(2 \times 10 \mathrm{~mL})$, water $(2 \times 10 \mathrm{~mL})$, brine $(2 \times 10 \mathrm{~mL})$. The extract was dried over $\mathrm{NaSO}_{4}$, filtered and concentrated in vacuo. The crude product was purified by column chromatography on silica gel (petroleum ether/ethyl acetate 10/1) to afford dibenzyl malonate $(7.77 \mathrm{~g}, 95 \%)$ as a colorless liquid.

\section{General procedure for preparation of $(\boldsymbol{R})$-Ir-Ligand ${ }^{[3]}$}

The preparation of $(\boldsymbol{R})$-Ir-Ligand described previously by Prof. Michael J. Krische was modified as follow: To a mixture of $[\mathrm{Ir}(\mathrm{cod}) \mathrm{Cl}]_{2}(10 \mathrm{mmol} \%),(R)$-Ligand $(20 \mathrm{mmol} \%), \mathrm{Cs}_{2} \mathrm{CO}_{3}(40 \mathrm{mmol} \%)$, 4-CN-3- $\mathrm{NO}_{2} \mathrm{BzOH}(40 \mathrm{mmol} \%)$ and allyl acetate $(50 \mathrm{mmol} \%)$ in a sealed tube under argon atmosphere was added THF $(4.0 \mathrm{~mL}, 0.05 \mathrm{M})$. The reaction mixture was stirred for $30 \mathrm{~min}$ at ambient temperature and heated for $1.5 \mathrm{hr}$ at $80^{\circ} \mathrm{C}$, at which point the reaction mixture was allowed to cool to ambient temperature. The reaction mixture was filtered through a celite and washed by DCM $(10 \mathrm{~mL})$. The filtrate was concentrated in vacuo and subjected to column chromatography (DCM: $\left.\mathrm{Et}_{2} \mathrm{O} 10: 1\right)$. The obtained gum-like product was dissolved in THF $(0.5 \mathrm{~mL})$, and precipitated upon rapid addition of HPLC grade hexanes $(7 \mathrm{~mL})$. A yellow precipitate formed, which was collected by filtration and dried under vacuum.

\section{Preparation of (rac)-Ir-Ligand}

The preparation of (rac)-Ir-Ligand was achieved by using the above procedure with BIPHEP to afford Ir-BIPHEP. 


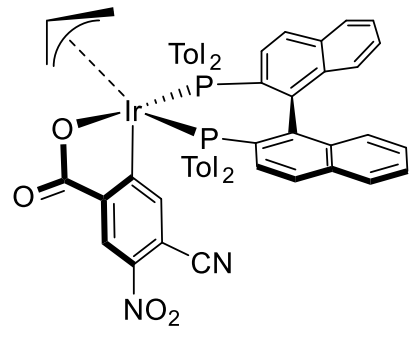

(R)-Ir-Tol-BINAP

cat-1

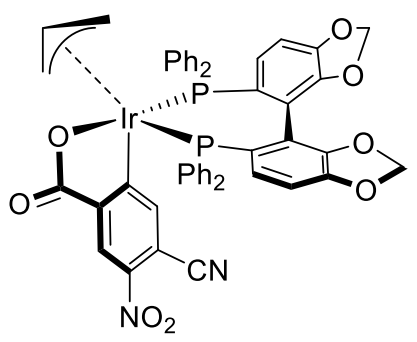

(R)-Ir-SEGPHOS

cat-4

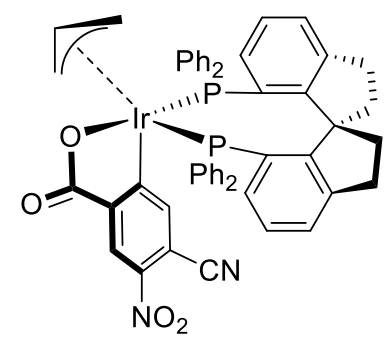

(R)-Ir-SDP

cat-2

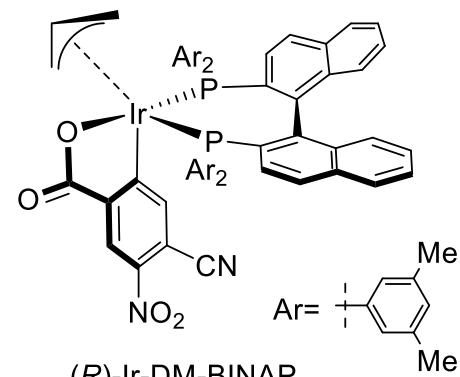

(R)-Ir-DM-BINAP

cat-5

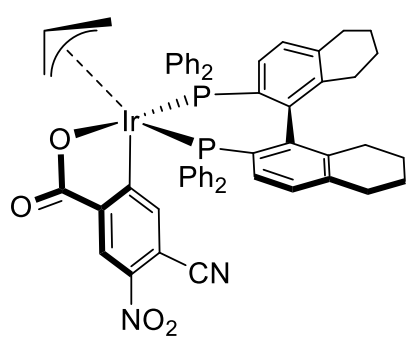

(R)-Ir- $\mathrm{H}_{8}$-BINAP

cat-3

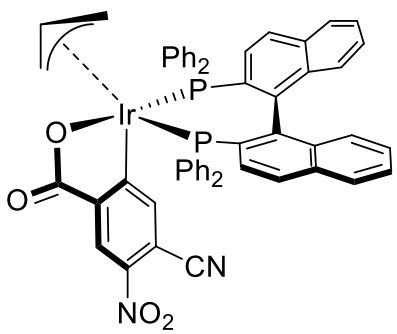

Ir-BIPHEP

\section{Characterization of new catalyst:}

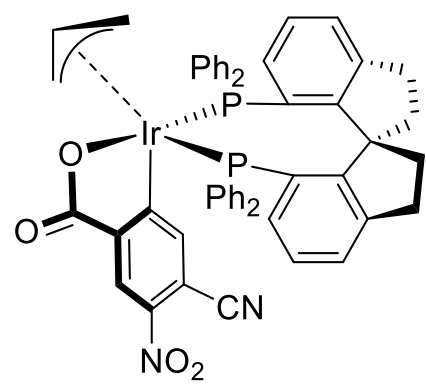

(R)-Ir-SDP

cat-2

The title compound was prepared according to the general procedure to afford cat-2 as yellow powder in $57 \%$ yield. (Afford $27 \mathrm{mg}$ of (R)-Ir-SDP by using $4.7 \mathrm{mmol} \%(R)$-SDP)

${ }^{1} \mathbf{H}$ NMR $\left(400 \mathrm{MHz}, \mathrm{CDCl}_{3}\right) \delta 8.42(\mathrm{dd}, \mathrm{J}=7.9,11.9 \mathrm{~Hz}, 1 \mathrm{H}), 8.23(\mathrm{dd}, \mathrm{J}=7.9,11.8 \mathrm{~Hz}, 1 \mathrm{H})$, $8.16(\mathrm{~d}, \mathrm{~J}=1.7 \mathrm{~Hz}, 1 \mathrm{H}), 7.82(\mathrm{t}, \mathrm{J}=8.3 \mathrm{~Hz}, 2 \mathrm{H}), 7.56-7.31(\mathrm{~m}, 12 \mathrm{H}), 7.18(\mathrm{br}, 3 \mathrm{H}), 7.12-7.00$ $(\mathrm{m}, 8 \mathrm{H}), 6.23(\mathrm{~d}, \mathrm{~J}=5.0 \mathrm{~Hz}, 2 \mathrm{H}), 4.30(\mathrm{br}, 1 \mathrm{H}), 3.24(\mathrm{~d}, \mathrm{~J}=6.1 \mathrm{~Hz}, 1 \mathrm{H}), 2.42(\mathrm{dq}, \mathrm{J}=, 9.5,18.9$ $\mathrm{Hz}, 2 \mathrm{H}), 1.95-1.71(\mathrm{~m}, 3 \mathrm{H}), 1.36-1.08$ (m, 3H), $1.16-1.06$ (m, 1H), 0.69 (q, J = $10.8 \mathrm{~Hz}$, 1H) ${ }^{13} \mathrm{C}$ NMR $\left(151 \mathrm{MHz}, \mathrm{CDCl}_{3}\right) \delta 179.7,162.5,161.8,150.0,149.9,149.2,149.2,147.4$, 
147.4, 146.0, 145.9, 143.5, 141.3, 138.5, 137.9, 137.1, 135.5, 134.0, 132.2, 131.6, 131.3, 130.8, $130.2,130.1,128.8,128.7,128.6,128.5,128.5,128.2,128.1,127.6,127.6,127.4,127.3,126.7$, 126.7, 126.1, 126.1, 123.6, 116.7, 107.1, 93.3, 66.3, 55.5, 55.2, 36.8, 36.1, 31.4, 29.9, 29.9, 29.7. ${ }^{31}$ P NMR $\left(162 \mathrm{MHz}, \mathrm{CDCl}_{3}\right) \delta-3.8(\mathrm{~d}, \mathrm{~J}=20.7 \mathrm{~Hz}),-7.7(\mathrm{~d}, \mathrm{~J}=19.6 \mathrm{~Hz}),-9.5(\mathrm{~d}, \mathrm{~J}=23.6$ $\mathrm{Hz}),-13.1(\mathrm{~d}, \mathrm{~J}=23.5 \mathrm{~Hz})$. HRMS $\left(\mathrm{ESI}^{+}\right): \mathrm{m} / z$ calcd for $\mathrm{C}_{52} \mathrm{H}_{41} \mathrm{IrN}_{2} \mathrm{O}_{4} \mathrm{P}_{2}[\mathrm{M}+\mathrm{H}]^{+}$1011.2220, found 1011.2220. MP: $208-214^{\circ} \mathrm{C}$ (decomposition)

\section{General procedure for enantioselective Ir-catalyzed allylic alkylation}

\section{with malonates}

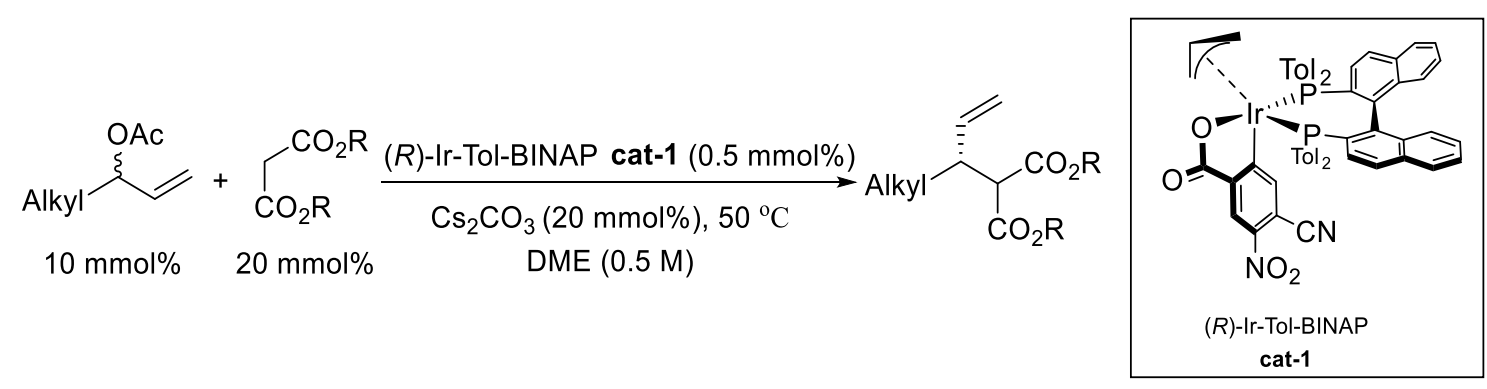

\section{General procedure:}

A pressure tube equipped with a magnetic stir bar was charged with cesium carbonate ( $20 \mathrm{mmol} \%$ ), $(R)$-Ir-Tol-BINAP $(0.5 \mathrm{mmol} \%)$, allylic acetate $(10 \mathrm{mmol} \%)$, dimethyl malonate / dibenzyl malonate (20 mmol\%), DME ( $0.5 \mathrm{M})$ was added. The tube was purged with argon for 1 minute. The tube was sealed with a PTFE lined cap and was placed in an oil bath at the indicated temperature and stirred for the indicated period of time. After reaching ambient temperature, the crude reaction mixture was directly subjected to flash column chromatography.<smiles>C=C[C@H](CCc1ccccc1)C(C(C)=O)C(C)=O</smiles>

3a

The compound was prepared according to the general procedure $\left(50{ }^{\circ} \mathrm{C}, 40 \mathrm{hr}\right)$. The crude reaction mixture was purified on silica gel (petroleum ether/ethyl acetate 20/1) to afford 3a as a colorless oil $(26.0 \mathrm{mg}, 94 \%)$. 
$\mathbf{R}_{f}=0.40$ (petroleum ether/ethyl acetate 10/1). ${ }^{1} \mathbf{H}$ NMR $\left(400 \mathrm{MHz}, \mathrm{CDCl}_{3}\right) \delta$ 7.36-7.32 (m, 2H), $7.24-7.21(\mathrm{~m}, 3 \mathrm{H}), 5.78(\mathrm{ddd}, J=9.8,9.9,16.8 \mathrm{~Hz}, 1 \mathrm{H}), 5.31-5.11(\mathrm{~m}, 2 \mathrm{H}), 3.78(\mathrm{~s}$, 3H), $3.75(\mathrm{~s}, 3 \mathrm{H}), 3.49$ (d, $J=8.7 \mathrm{~Hz}, 1 \mathrm{H}), 2.90(\mathrm{~m}, 1 \mathrm{H}), 2.68(\mathrm{~m}, 1 \mathrm{H}), 2.51(\mathrm{~m}, 1 \mathrm{H}), 1.87(\mathrm{~m}$, 1H), $1.70(\mathrm{~m}, 1 \mathrm{H}) .{ }^{13} \mathrm{C}$ NMR (100 MHz, $\left.\mathrm{CDCl}_{3}\right) \delta 168.7,168.5,141.7,137.7,128.4,128.4$, 125.9, 118.2, 56.8, 52.5, 52.3, 43.9, 34.1, 33.3 ppm. FT-IR (KBr): 3083, 3063, 3026, 2979, 2952, 2858, 1755, 1738, 1641, 1603, 1454, 1435, 1298, 1265, 1246, 1199, 1153, 925, 748, 700 $\mathrm{cm}^{-1}$. HRMS $\left(\mathrm{ESI}^{+}\right): \mathrm{m} / \mathrm{z}$ calcd for $\mathrm{C}_{16} \mathrm{H}_{20} \mathrm{O}_{4} \mathrm{Na}[\mathrm{M}+\mathrm{Na}]^{+} 299.1254$, found 299.1259. $[\boldsymbol{\alpha}]^{24.3}{ }_{\mathrm{D}}=$ $11.53\left(c=0.59, \mathrm{CHCl}_{3}\right)$. HPLC: Daicel Chiralpak IC, $n$-hexane $/ i-\mathrm{PrOH}=95 / 5,0.6 \mathrm{~mL} / \mathrm{min}$, $25{ }^{\circ} \mathrm{C}, 210 \mathrm{~nm} ; 97: 3 \operatorname{er}\left[\mathrm{t}_{1}\right.$ (minor) $=11.044 \mathrm{~min}, \mathrm{t}_{2}$ (major) $\left.=11.602 \mathrm{~min}\right]$.

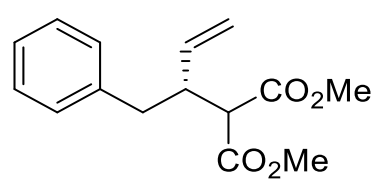

$3 \mathbf{b}$

The compound was prepared according to the general procedure $\left(70{ }^{\circ} \mathrm{C}, 40 \mathrm{hr}\right)$. The crude reaction mixture was purified on silica gel (petroleum ether/ethyl acetate 20/1) to afford $\mathbf{3 b}$ as a colorless oil (22.0 mg, 84\%).

$\mathbf{R}_{f}=0.43$ (petroleum ether/ethyl acetate 10/1). ${ }^{1} \mathbf{H}$ NMR $\left(400 \mathrm{MHz}, \mathrm{CDCl}_{3}\right) \delta$ 7.29-7.25 (m, 2H), 7.21-7.14 (m, 3H), $5.74(\mathrm{ddd}, J=9.6,9.717 .1 \mathrm{~Hz}, 1 \mathrm{H}), 5.01-4.91(\mathrm{~m}, 2 \mathrm{H}), 3.73(\mathrm{~s}, 3 \mathrm{H})$, $3.70(\mathrm{~s}, 3 \mathrm{H}), 3.46(\mathrm{~d}, J=8.0 \mathrm{~Hz}, 1 \mathrm{H}), 3.10(\mathrm{~m}, 1 \mathrm{H}), 2.86(\mathrm{dd}, J=5.4,13.5 \mathrm{~Hz}, 1 \mathrm{H}), 2.65(\mathrm{dd}$, $J=8.8,13.5 \mathrm{~Hz}, 1 \mathrm{H}) .{ }^{13} \mathbf{C} \mathbf{~ N M R}\left(100 \mathrm{MHz}, \mathrm{CDCl}_{3}\right) \delta 168.8,168.5,138.9,137.2,129.4,128.3$, 126.3, 117.6, 55.7, 52.5, 52.3, 45.6, 38.9 ppm. FT-IR (KBr): 3084, 3064, 3028, 3003, 2953, 2920, 2848, 1754, 1737, 1641, 1603, 1496, 1454, 1435, 1320, 1257, 1201, 1157, 925, 747, 702 $\mathrm{cm}^{-1}$. HRMS (ESI $\left.{ }^{+}\right): m / z$ calcd for $\mathrm{C}_{15} \mathrm{H}_{18} \mathrm{O}_{4} \mathrm{Na}[\mathrm{M}+\mathrm{Na}]^{+} 285.1097$, found 285.1097. $[\alpha]^{24.3} \mathrm{D}=$ $10.88\left(c=0.47, \mathrm{CHCl}_{3}\right)$. HPLC: Daicel Chiralpak IC, $n$-hexane $/ i$-PrOH $=99 / 1,0.7 \mathrm{~mL} / \mathrm{min}$, $25^{\circ} \mathrm{C}, 210 \mathrm{~nm} ; 94: 6 \operatorname{er}\left[\mathrm{t}_{1}\right.$ (minor) $=17.527 \mathrm{~min}, \mathrm{t}_{2}$ (major) $\left.=18.362 \mathrm{~min}\right]$.

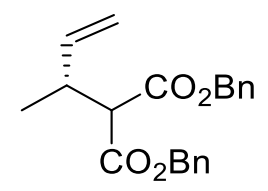

3c 
The compound was prepared according to the general procedure $\left(40{ }^{\circ} \mathrm{C}, 40 \mathrm{hr}\right)$. The crude reaction mixture was purified on silica gel (petroleum ether/ethyl acetate 20/1) to afford $\mathbf{3 c}$ as a colorless oil (31.1 mg, 92\%).

$\mathbf{R}_{f}=0.53$ (petroleum ether/ethyl acetate 10/1). ${ }^{1} \mathbf{H}$ NMR $\left(400 \mathrm{MHz}, \mathrm{CDCl}_{3}\right) \delta 7.49-7.08(\mathrm{~m}$, 10H), 5.74 (ddd, J = 8.8, 10.2, $16.9 \mathrm{~Hz}, 1 \mathrm{H}), 5.15(\mathrm{~s}, 2 \mathrm{H}), 5.11(\mathrm{~s}, 2 \mathrm{H}), 5.03(\mathrm{~d}, \mathrm{~J}=16.9 \mathrm{~Hz}$, $1 \mathrm{H}), 4.96(\mathrm{~d}, \mathrm{~J}=10.2 \mathrm{~Hz}, 1 \mathrm{H}), 3.40(\mathrm{~d}, \mathrm{~J}=8.8 \mathrm{~Hz}, 1 \mathrm{H}), 2.98(\mathrm{qd}, \mathrm{J}=3.3,7.5 \mathrm{~Hz}, 1 \mathrm{H}), 1.08(\mathrm{~d}$, $\mathrm{J}=8.8 \mathrm{~Hz}, 3 \mathrm{H}) .{ }^{13} \mathbf{C} \mathbf{N M R}\left(100 \mathrm{MHz}, \mathrm{CDCl}_{3}\right) \delta 168.1,168.0,139.6,135.4,135.4,128.6,128.5$, 128.4, 128.3, 128.3, 128.3, 115.6, 67.1, 67.0, 57.7, 38.1, 17.9 ppm. FT-IR (KBr): 3093, 3066, 3033, 2966, 2936, 2883, 1752, 1733, 1641, 1498, 1455, 1376, 1336, 1317, 1266, 1216, 1144 , 920, 748, $697 \mathrm{~cm}^{-1}$. HRMS $\left(\right.$ ESI $\left.^{+}\right): \mathrm{m} / z$ calcd for $\mathrm{C}_{21} \mathrm{H}_{22} \mathrm{O}_{4} \mathrm{Na}[\mathrm{M}+\mathrm{Na}]^{+} 361.1410$, found 361.1407. $[\alpha]^{24.5}{ }_{\mathrm{D}}=5.22\left(c=0.27, \mathrm{CHCl}_{3}\right)$. HPLC: Daicel Chiralpak AD-H, $n$-hexane/i-PrOH $=99 / 1,1.0 \mathrm{~mL} / \mathrm{min}, 25{ }^{\circ} \mathrm{C}, 210 \mathrm{~nm} ; 93: 7 \operatorname{er}\left[\mathrm{t}_{1}(\right.$ minor $)=13.499 \mathrm{~min}, \mathrm{t}_{2}$ (major) $\left.=14.53 \mathrm{~min}\right]$.

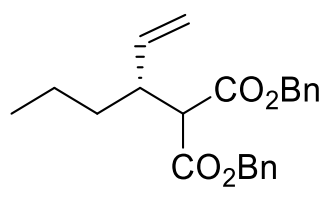

$3 d$

The compound was prepared according to the general procedure $\left(50{ }^{\circ} \mathrm{C}, 40 \mathrm{hr}\right)$. The crude reaction mixture was purified on silica gel (petroleum ether/ethyl acetate 20/1) to afford $\mathbf{3 d}$ as a colorless oil (35.1 mg, 96\%).

$\mathbf{R}_{f}=0.58$ (petroleum ether/ethyl acetate 10/1). ${ }^{1} \mathbf{H}$ NMR $\left(400 \mathrm{MHz}, \mathrm{CDCl}_{3}\right) \delta$ 7.35-7.28 (m, $10 \mathrm{H}), 5.63(\mathrm{ddd}, \mathrm{J}=8.8,9.8,17.0 \mathrm{~Hz}, 1 \mathrm{H}), 5.15(\mathrm{~s}, 2 \mathrm{H}), 5.10(\mathrm{~s}, 2 \mathrm{H}), 5.05-4.78(\mathrm{~m}, 2 \mathrm{H}), 3.47$ $(\mathrm{d}, \mathrm{J}=8.8 \mathrm{~Hz}, 1 \mathrm{H}), 2.81(\mathrm{td}, \mathrm{J}=3.3,8.8 \mathrm{~Hz}, 1 \mathrm{H}), 1.53-1.00(\mathrm{~m}, 4 \mathrm{H}), 0.82(\mathrm{t}, \mathrm{J}=6.9 \mathrm{~Hz}, 3 \mathrm{H})$. ${ }^{13} \mathrm{C}$ NMR $\left(100 \mathrm{MHz}, \mathrm{CDCl}_{3}\right) \delta 168.1,168.0,138.0,135.4,135.4,128.6,128.5,128.4,128.3$, 128.3, 128.3, 117.5, 67.1, 67.0, 57.0, 44.0, 34.4, 20.1, 13.8 ppm. FT-IR (KBr): 3067, 3034, 2958, 2932, 2872, 1754, 1734, 1641, 1498, 1455, 1377, 1257, 1227, 1141, 1000, 922, 749, 697 $\mathrm{cm}^{-1}$. HRMS $\left(\mathrm{ESI}^{+}\right): \mathrm{m} / z$ calcd for $\mathrm{C}_{23} \mathrm{H}_{26} \mathrm{O}_{4} \mathrm{Na}[\mathrm{M}+\mathrm{Na}]^{+} 389.1723$, found 389.1717. $[\boldsymbol{\alpha}]^{24.1} \mathrm{D}=$ -4.37 ( $\left.c=0.75, \mathrm{CHCl}_{3}\right)$. HPLC: Daicel Chiralpak IC, $n$-hexane $/ i-\mathrm{PrOH}=99 / 1,1.0 \mathrm{~mL} / \mathrm{min}$, $25^{\circ} \mathrm{C}, 210 \mathrm{~nm} ; 94: 6$ er $\left[\mathrm{t}_{1}\right.$ (minor) $=12.688 \mathrm{~min}, \mathrm{t}_{2}$ (major) $\left.=13.81 \mathrm{~min}\right]$. 


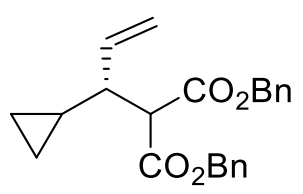

$3 e$

The compound was prepared according to the general procedure $\left(50{ }^{\circ} \mathrm{C}, 40 \mathrm{hr}\right)$. The crude reaction mixture was purified on silica gel (petroleum ether/ethyl acetate 20/1) to afford 3e as a colorless oil (34.6 mg, 95\%).

$\mathbf{R}_{f}=0.53$ (petroleum ether/ethyl acetate 10/1). ${ }^{1} \mathbf{H}$ NMR $\left(400 \mathrm{MHz}, \mathrm{CDCl}_{3}\right) \delta$ 7.35-7.30 (m, $10 \mathrm{H}), 5.82(\mathrm{ddd}, \mathrm{J}=8.0,10.3,17.7 \mathrm{~Hz}, 1 \mathrm{H}), 5.16-5.13(\mathrm{~m}, 4 \mathrm{H}), 5.10-5.00(\mathrm{~m}, 2 \mathrm{H}), 3.64(\mathrm{~d}, \mathrm{~J}$ $=8.4 \mathrm{~Hz}, 1 \mathrm{H}), 2.16(\mathrm{ddd}, \mathrm{J}=8.0,8.4,8.5 \mathrm{~Hz}, 1 \mathrm{H}), 0.95(\mathrm{~m}, 1 \mathrm{H}), 0.45(\mathrm{~m}, 1 \mathrm{H}), 0.40(\mathrm{~m}, 1 \mathrm{H})$, $0.20(\mathrm{td}, \mathrm{J}=4.9,9.4 \mathrm{~Hz}, 1 \mathrm{H}), 0.10(\mathrm{td}, \mathrm{J}=4.9,9.4 \mathrm{~Hz}, 1 \mathrm{H}) .{ }^{13} \mathbf{C} \mathbf{~ N M R}\left(100 \mathrm{MHz}, \mathrm{CDCl}_{3}\right) \delta$ 168.1, 167.9, 137.4, 135.4, 135.3, 128.6, 128.5, 128.4, 128.4, 128.3, 128.3, 116.5, 67.1, 67.0, 57.0, 48.3, 13.5, 4.8, 3.5 ppm. FT-IR (KBr): 3078, 3067, 3033, 3004, 2956, 2849, 1754, 1734 , 1640, 1498, 1455, 1377, 1299, 1261, 1216, 1144, 1021, 1001, 922, 750, $698 \mathrm{~cm}^{-1}$. HRMS $\left(\mathrm{ESI}^{+}\right): \mathrm{m} / z$ calcd for $\mathrm{C}_{23} \mathrm{H}_{24} \mathrm{O}_{4} \mathrm{Na}[\mathrm{M}+\mathrm{Na}]^{+} 387.1567$, found 387.1558. $[\alpha]^{24.5} \mathrm{D}=-19.39(c=$ 0.75, $\mathrm{CHCl}_{3}$ ). HPLC: Daicel Chiralpak IC, $n$-hexane $/ i$-PrOH = 99/1, $1.0 \mathrm{~mL} / \mathrm{min}, 25{ }^{\circ} \mathrm{C}, 210$ $\mathrm{nm} ;$ 95:5 $\operatorname{er}\left[\mathrm{t}_{1}(\right.$ minor $)=10.809 \mathrm{~min}, \mathrm{t}_{2}($ major $\left.)=12.038 \mathrm{~min}\right]$.

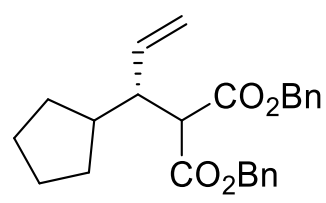

$3 f$

The compound was prepared according to the general procedure $\left(50{ }^{\circ} \mathrm{C}, 40 \mathrm{hr}\right)$. The crude reaction mixture was purified on silica gel (petroleum ether/ethyl acetate 20/1) to afford $\mathbf{3 f}$ as a colorless oil (36.9 mg, 94\%).

$\mathbf{R}_{f}=0.58$ (petroleum ether/ethyl acetate 10/1). ${ }^{1} \mathbf{H} \mathbf{~ N M R}\left(400 \mathrm{MHz}, \mathrm{CDCl}_{3}\right) \delta 7.32(\mathrm{~m}, 10 \mathrm{H})$, $5.82(\mathrm{ddd}, \mathrm{J}=9.8,10.1,16.9 \mathrm{~Hz}, 1 \mathrm{H}), 5.19-5.06(\mathrm{~m}, 4 \mathrm{H}), 5.07-4.91(\mathrm{~m}, 2 \mathrm{H}), 3.65(\mathrm{~d}, \mathrm{~J}=$ $7.7 \mathrm{~Hz}, 1 \mathrm{H}), 2.71(\mathrm{ddd}, \mathrm{J}=7.3,7.7,9.8 \mathrm{~Hz}, 1 \mathrm{H}), 1.85$ (ddt, J = 7.4, 9.7, 17.4 Hz, 1H), 1.73 1.49 (m, 4H), 1.48-1.42 (m, 2H), $1.24-1.01(\mathrm{~m}, 2 \mathrm{H}) .{ }^{13} \mathrm{C}$ NMR (100 MHz, $\left.\mathrm{CDCl}_{3}\right) \delta 168.5$, 168.0, 136.3, 135.5, 135.4, 128.5, 128.5, 128.4, 128.4, 128.3, 128.3, 118.0, 67.1, 66.9, 55.9, 49.2, 41.7, 30.6, 29.5, 25.1, 25.0 ppm. FT-IR (KBr): 3067, 3033, 3007, 2953, 2868, 1754, 1733 , 1638, 1498, 1377, 1344, 1260, 1216, 1149, 1121, 1001, 921, 750, $697 \mathrm{~cm}^{-1}$. HRMS $\left(\mathrm{ESI}^{+}\right): \mathrm{m} / \mathrm{z}$ 
calcd for $\mathrm{C}_{25} \mathrm{H}_{28} \mathrm{O}_{4} \mathrm{Na}[\mathrm{M}+\mathrm{Na}]^{+}$415.1880, found 415.1882. $[\alpha]^{25.1} \mathrm{D}=-4.11\left(c=0.55, \mathrm{CHCl}_{3}\right)$. HPLC: Daicel Chiralpak IC, $n$-hexane $/ i-\mathrm{PrOH}=99 / 1,1.0 \mathrm{~mL} / \mathrm{min}, 25^{\circ} \mathrm{C}, 210 \mathrm{~nm} ; 93: 7$ er $\left[\mathrm{t}_{1}\right.$ $($ minor $)=12.244 \mathrm{~min}, \mathrm{t}_{2}($ major $\left.)=18.316 \mathrm{~min}\right]$.

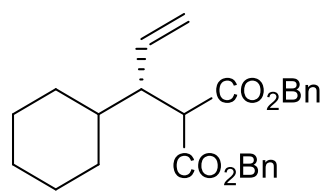

$3 g$

The compound was prepared according to the general procedure $\left(50{ }^{\circ} \mathrm{C}, 40 \mathrm{hr}\right)$. The crude reaction mixture was purified on silica gel (petroleum ether/ethyl acetate 20/1) to afford $\mathbf{3 g}$ as a colorless oil (35.8 mg, 88\%).

$\mathbf{R}_{f}=0.58$ (petroleum ether/ethyl acetate 10/1). ${ }^{1} \mathbf{H}$ NMR (400 MHz, $\left.\mathrm{CDCl}_{3}\right) \delta$ 7.37-7.28 (m, $10 \mathrm{H}), 5.71(\mathrm{ddd}, \mathrm{J}=9.5,10.1,17.0 \mathrm{~Hz}, 1 \mathrm{H}), 5.15(\mathrm{~d}, \mathrm{~J}=2.2 \mathrm{~Hz}, 2 \mathrm{H}), 5.10(\mathrm{~d}, \mathrm{~J}=2.6 \mathrm{~Hz}, 2 \mathrm{H})$, $5.06-4.86(\mathrm{~m}, 2 \mathrm{H}), 3.70(\mathrm{~d}, \mathrm{~J}=9.1 \mathrm{~Hz}, 1 \mathrm{H}), 2.66(\mathrm{ddd}, \mathrm{J}=9.1,9.4,9.5 \mathrm{~Hz}, 1 \mathrm{H}), 1.73-1.61$ $(\mathrm{m}, 3 \mathrm{H}), 1.59(\mathrm{~d}, \mathrm{~J}=9.1 \mathrm{~Hz}, 1 \mathrm{H}), 1.53(\mathrm{~d}, \mathrm{~J}=12.0 \mathrm{~Hz}, 1 \mathrm{H}), 1.31(\mathrm{~m}, 1 \mathrm{H}), 1.17-0.96(\mathrm{~m}, 4 \mathrm{H})$, $0.85(\mathrm{~m}, 1 \mathrm{H}) .{ }^{13} \mathbf{C}$ NMR $\left(100 \mathrm{MHz}, \mathrm{CDCl}_{3}\right) \delta 168.5,168.1,135.7,135.5,135.4,128.6,128.5$, 128.4, 128.4, 128.3, 67.1, 66.9, 54.4, 50.2, 38.9, 31.5, 28.5, 26.3, 26.2 ppm. FT-IR (KBr): 3067, 3033, 3009, 2926, 2852, 1755, 1734, 1638, 1498, 1454, 1376, 1303, 1259, 1217, 1189, 1140, 1001, 921, 750, $698 \mathrm{~cm}^{-1}$. HRMS (ESI $)^{+}: \mathrm{m} / z$ calcd for $\mathrm{C}_{26} \mathrm{H}_{30} \mathrm{O}_{4} \mathrm{Na}[\mathrm{M}+\mathrm{Na}]^{+} 429.2036$, found 429.2035. $[\alpha]^{24.0}{ }_{\mathrm{D}}=-5.43\left(c=0.66, \mathrm{CHCl}_{3}\right)$. HPLC: Daicel Chiralpak IC, $n$-hexane $/ i-\mathrm{PrOH}=$ 99/1, $1.0 \mathrm{~mL} / \mathrm{min}, 25^{\circ} \mathrm{C}, 210 \mathrm{~nm}$; $93: 7$ er $\left[\mathrm{t}_{1}\right.$ (minor) $=11.719 \mathrm{~min}, \mathrm{t}_{2}$ (major) $\left.=15.556 \mathrm{~min}\right]$.

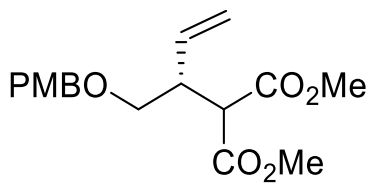

$3 \mathrm{~h}$

The compound was prepared according to the general procedure $\left(50{ }^{\circ} \mathrm{C}, 40 \mathrm{hr}\right)$. The crude reaction mixture was purified on silica gel (petroleum ether/ethyl acetate 30/1) to afford $\mathbf{3 h}$ as a colorless oil (30.0 mg, 93\%).

$\mathbf{R}_{f}=0.25$ (petroleum ether/ethyl acetate 10/1). ${ }^{1} \mathbf{H} \mathbf{N M R}\left(400 \mathrm{MHz}, \mathrm{CDCl}_{3}\right) \delta 7.23(\mathrm{~d}, \mathrm{~J}=8.5$ $\mathrm{Hz}, 2 \mathrm{H}), 6.87(\mathrm{~d}, \mathrm{~J}=8.6 \mathrm{~Hz}, 2 \mathrm{H}), 5.83(\mathrm{ddd}, J=9.5,9.7,17.3 \mathrm{~Hz}, 1 \mathrm{H}), 5.18-5.10(\mathrm{~m}, 2 \mathrm{H})$, 
$4.40(\mathrm{~s}, 2 \mathrm{H}), 3.80(\mathrm{~s}, 3 \mathrm{H}), 3.69(\mathrm{~m}, 1 \mathrm{H}), 3.67(\mathrm{~s}, 3 \mathrm{H}), 3.65(\mathrm{~s}, 3 \mathrm{H}), 3.56-3.47(\mathrm{~m}, 2 \mathrm{H}), 3.13(\mathrm{~m}$, 1H). ${ }^{13} \mathrm{C}$ NMR $\left(100 \mathrm{MHz}, \mathrm{CDCl}_{3}\right) \delta 168.8,168.7,159.2,135.5,130.2,129.3,118.1,113.7$, 72.8, 55.3, 53.3, 52.4, 52.3, 44.0 ppm. FT-IR (KBr): 3077, 3001, 2953, 2915, 2860, 2837, 1753 , 1736, 1612, 1514, 1456, 1435, 1360, 1302, 1248, 1195, 1174, 1099, 1033, 926, 846, $820 \mathrm{~cm}^{-1}$. HRMS $\left(\mathrm{ESI}^{+}\right): \mathrm{m} / z$ calcd for $\mathrm{C}_{17} \mathrm{H}_{22} \mathrm{O}_{6} \mathrm{Na}[\mathrm{M}+\mathrm{Na}]^{+} 345.1309$, found 345.1310. $[\boldsymbol{\alpha}]^{24.2} \mathrm{D}=31.65$ $\left(c=0.23, \mathrm{CHCl}_{3}\right)$. HPLC: Daicel Chiralpak IC, $n$-hexane $/ i-\mathrm{PrOH}=80 / 20,1.0 \mathrm{~mL} / \mathrm{min}, 25^{\circ} \mathrm{C}$, $210 \mathrm{~nm} ; 94: 6 \operatorname{er}\left[\mathrm{t}_{1}(\right.$ minor $)=7.506 \mathrm{~min}, \mathrm{t}_{2}($ major $\left.)=8.34 \mathrm{~min}\right]$.

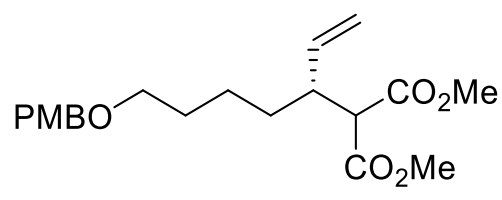

$3 \mathbf{i}$

The compound was prepared according to the general procedure $\left(50{ }^{\circ} \mathrm{C}, 40 \mathrm{hr}\right)$. The crude reaction mixture was purified on silica gel (petroleum ether/ethyl acetate 20/1) to afford $\mathbf{3 i}$ as a colorless oil $(33.8 \mathrm{mg}, 93 \%)$.

$\mathbf{R}_{f}=0.26$ (petroleum ether/ethyl acetate 10/1). ${ }^{1} \mathbf{H}$ NMR $\left(400 \mathrm{MHz}, \mathrm{CDCl}_{3}\right) \delta 7.25(\mathrm{~d}, \mathrm{~J}=8.5$ $\mathrm{Hz}, 2 \mathrm{H}), 6.87(\mathrm{~d}, \mathrm{~J}=8.6 \mathrm{~Hz}, 2 \mathrm{H}), 5.62(\mathrm{ddd}, J=9.6,9.7,16.8 \mathrm{~Hz}, 1 \mathrm{H}), 5.11-5.06(\mathrm{~m}, 2 \mathrm{H})$, 4.41 (s, 2H), 3.80 (s, 3H), 3.72 (s, 3H), 3.68 (s, 3H), $3.43-3.37$ (m, 3H), 2.76 (dd, $J=3.2,9.2$ $\mathrm{Hz}, 1 \mathrm{H}), 1.58(\mathrm{dt}, J=6.6,19.8 \mathrm{~Hz}, 2 \mathrm{H}), 1.47-1.38(\mathrm{~m}, 2 \mathrm{H}), 1.36-1.23(\mathrm{~m}, 2 \mathrm{H}) .{ }^{13} \mathbf{C} \mathbf{N M R}(100$ $\left.\mathrm{MHz}, \mathrm{CDCl}_{3}\right) \delta 168.8,168.6,159.1,137.9,130.7,129.2,117.6,113.8,72.5,70.8,69.9,56.9$, 55.3, 52.4, 52.3, 44.2, 32.1, 29.4, 23.7 ppm. FT-IR (KBr): 3076, 3000, 2950, 2936, 2859, 1756, 1737, 1613, 1513, 1458, 1435, 1360, 1301, 1247, 1207, 1171, 1159, 1098, 1034, 923, 821, 757 $\mathrm{cm}^{-1}$. HRMS $\left(\mathrm{ESI}^{+}\right): \mathrm{m} / \mathrm{z}$ calcd for $\mathrm{C}_{20} \mathrm{H}_{28} \mathrm{O}_{6} \mathrm{Na}[\mathrm{M}+\mathrm{Na}]^{+} 387.1778$, found 387.1778. $[\alpha]^{24.1} \mathrm{D}=$ $4.62\left(c=0.82, \mathrm{CHCl}_{3}\right)$. HPLC: Daicel Chiralpak IC, $n$-hexane $/ i-\mathrm{PrOH}=80 / 20,1.0 \mathrm{~mL} / \mathrm{min}$, $25^{\circ} \mathrm{C}, 210 \mathrm{~nm} ; 96: 4 \operatorname{er}\left[\mathrm{t}_{1}\right.$ (major) $=5.358 \mathrm{~min}, \mathrm{t}_{2}($ minor $\left.)=5.752 \mathrm{~min}\right]$.

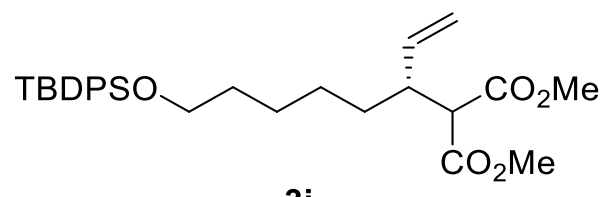

$3 \mathbf{j}$

The compound was prepared according to the general procedure $\left(50{ }^{\circ} \mathrm{C}, 40 \mathrm{hr}\right)$. The crude reaction mixture was purified on silica gel (petroleum ether/ethyl acetate 20/1) to afford $\mathbf{3 j}$ as a colorless oil $(47.7 \mathrm{mg}, 96 \%)$. 
$\mathbf{R}_{f}=0.50$ (petroleum ether/ethyl acetate 10/1). ${ }^{1} \mathbf{H}$ NMR $\left(400 \mathrm{MHz}, \mathrm{CDCl}_{3}\right) \delta$ 7.68-7.66 (m, $4 \mathrm{H}), 7.44-7.36(\mathrm{~m}, 6 \mathrm{H}), 5.62(\mathrm{ddd}, J=9.5,9.7,16.7 \mathrm{~Hz}, 1 \mathrm{H}), 5.10-5.06(\mathrm{~m}, 2 \mathrm{H}), 3.74(\mathrm{~s}, 3 \mathrm{H})$, $3.73(\mathrm{~s}, 3 \mathrm{H}), 3.66(\mathrm{t}, J=6.4,2 \mathrm{H}), 3.39(\mathrm{~d}, J=8.9 \mathrm{~Hz}, 1 \mathrm{H}), 2.75(\mathrm{qd}, J=3.5,9.2 \mathrm{~Hz}, 1 \mathrm{H}), 1.57$ $-1.52(\mathrm{~m}, 2 \mathrm{H}), 1.45-1.21(\mathrm{~m}, 6 \mathrm{H}), 1.05(\mathrm{~s}, 9 \mathrm{H}) .{ }^{13} \mathbf{C} \mathbf{N M R}\left(100 \mathrm{MHz}, \mathrm{CDCl}_{3}\right) \delta$ 168.8, 168.6, 138.1, 135.6, 134.1, 129.5, 127.6, 117.5, 63.9, 56.9, 52.4, 52.2, 44.2, 32.4, 32.3, 26.9, 26.8, 25.6, 19.2 ppm. FT-IR (KBr): 3071, 3049, 3031, 2998, 2950, 2932, 2896, 2858, 1758, 1739, 1641, 1589, 1486, 1472, 1461, 1389, 1259, 1246, 1195, 1111, 1011, 998, 921, 823, 741, 704 $\mathrm{cm}^{-1}$. HRMS $\left(\mathrm{ESI}^{+}\right): \mathrm{m} / z$ calcd for $\mathrm{C}_{29} \mathrm{H}_{40} \mathrm{O}_{5} \mathrm{SiNa}[\mathrm{M}+\mathrm{Na}]^{+} 519.2537$, found $519.2538 .[\alpha]^{23.5} \mathrm{D}$ $=2.72\left(c=1.43, \mathrm{CHCl}_{3}\right)$. HPLC: Daicel Chiralpak IC, $n$-hexane $/ i-\mathrm{PrOH}=99 / 1,1.0 \mathrm{~mL} / \mathrm{min}$, $25^{\circ} \mathrm{C}, 210 \mathrm{~nm} ; 94: 6 \operatorname{er}\left[\mathrm{t}_{1}\right.$ (minor) $=7.331 \mathrm{~min}, \mathrm{t}_{2}$ (major) $\left.=8.145 \mathrm{~min}\right]$.

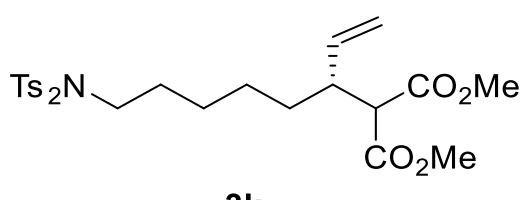

$3 k$

The compound was prepared according to the general procedure $\left(70{ }^{\circ} \mathrm{C}, 40 \mathrm{hr}\right)$. The crude reaction mixture was purified on silica gel (petroleum ether/ethyl acetate 7/1) to afford $\mathbf{3 k}$ as a colorless oil (50.4 mg, 89\%).

$\mathbf{R}_{f}=0.22$ (petroleum ether/ethyl acetate 5/1). ${ }^{1} \mathbf{H}$ NMR (400 MHz, $\left.\mathrm{CDCl}_{3}\right) \delta 7.89(\mathrm{~d}, \mathrm{~J}=8.1$ $\mathrm{Hz}, 4 \mathrm{H}), 7.33(\mathrm{~d}, \mathrm{~J}=8.1 \mathrm{~Hz}, 4 \mathrm{H}), 5.59(\mathrm{ddd}, \mathrm{J}=9.3,9.5,17.4 \mathrm{~Hz}, 1 \mathrm{H}), 5.07$ (dd, J = 2.2, 9.5 $\mathrm{Hz}, 2 \mathrm{H}), 3.72(\mathrm{~s}, 3 \mathrm{H}), 3.68(\mathrm{~s}, 3 \mathrm{H}), 3.65-3.46(\mathrm{~m}, 2 \mathrm{H}), 3.36(\mathrm{~d}, \mathrm{~J}=8.9 \mathrm{~Hz}, 1 \mathrm{H}), 2.73(\mathrm{tt}, \mathrm{J}=$ 4.4, $8.9 \mathrm{~Hz}, 1 \mathrm{H}), 2.45(\mathrm{~s}, 6 \mathrm{H}), 1.64(\mathrm{~d}, \mathrm{~J}=8.6 \mathrm{~Hz}, 2 \mathrm{H}), 1.37(\mathrm{~m}, 1 \mathrm{H}), 1.35-1.06(\mathrm{~m}, 5 \mathrm{H}) .{ }^{13} \mathrm{C}$ NMR $\left(100 \mathrm{MHz}, \mathrm{CDCl}_{3}\right) \delta 168.7,168.5,144.8,137.9,137.2,129.7,128.2,117.7,56.9,52.5$, 52.3, 49.2, 44.1, 32.0, 29.6, 26.4, 26.2, 21.7 ppm. FT-IR (KBr): 3071, 3030, 3003, 2951, 2860 , 1755, 1737, 1597, 1493, 1462, 1435, 1370, 1352, 1291, 1164, 1085, 923, 853, $723 \mathrm{~cm}^{-1}$. HRMS $\left(\mathrm{ESI}^{+}\right): \mathrm{m} / z$ calcd for $\mathrm{C}_{27} \mathrm{H}_{35} \mathrm{NO}_{8} \mathrm{~S}_{2} \mathrm{Na}[\mathrm{M}+\mathrm{Na}]^{+} 588.1696$, found 588.1705 . $[\alpha]^{24.3} \mathrm{D}=3.28(c=$ 1.14, $\mathrm{CHCl}_{3}$ ). HPLC: Daicel Chiralpak AD-H, $n$-hexane $/ i-\mathrm{PrOH}=90 / 10,0.7 \mathrm{~mL} / \mathrm{min}, 25^{\circ} \mathrm{C}$, $210 \mathrm{~nm} ; 97: 3 \operatorname{er}\left[\mathrm{t}_{1}(\right.$ minor $)=60.212 \mathrm{~min}, \mathrm{t}_{2}($ major $\left.)=63.159 \mathrm{~min}\right]$.

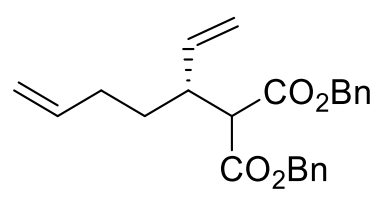

31 
The compound was prepared according to the general procedure $\left(50{ }^{\circ} \mathrm{C}, 40 \mathrm{hr}\right)$. The crude reaction mixture was purified on silica gel (petroleum ether/ethyl acetate 20/1) to afford $\mathbf{3 l}$ as a colorless oil (32.5 mg, 86\%).

$\mathbf{R}_{f}=0.55$ (petroleum ether/ethyl acetate 10/1). ${ }^{1} \mathbf{H}$ NMR $\left(400 \mathrm{MHz}, \mathrm{CDCl}_{3}\right) \delta 7.31(\mathrm{~m}, 10 \mathrm{H})$, 5.76 - $5.66(\mathrm{ddd}, \mathrm{J}=9.1,9.4,9.5 \mathrm{~Hz}, 1 \mathrm{H}), 5.60(\mathrm{~m}, 1 \mathrm{H}), 5.15(\mathrm{~s}, 2 \mathrm{H}), 5.10(\mathrm{~s}, 2 \mathrm{H}), 5.07-5.00$ $(\mathrm{m}, 2 \mathrm{H}), 4.99-4.87(\mathrm{~m}, 2 \mathrm{H}), 3.48(\mathrm{~d}, \mathrm{~J}=8.6 \mathrm{~Hz}, 1 \mathrm{H}), 2.83(\mathrm{td}, \mathrm{J}=3.6,9.4 \mathrm{~Hz}, 1 \mathrm{H}), 2.08$ (ddt, $\mathrm{J}=5.4,9.6,15.1 \mathrm{~Hz}, 1 \mathrm{H}), 1.94(\mathrm{ddt}, \mathrm{J}=5.4,7.5,14.9 \mathrm{~Hz}, 1 \mathrm{H}), 1.53(\mathrm{~m}, 1 \mathrm{H}), 1.37(\mathrm{~m}, 1 \mathrm{H}) .{ }^{13} \mathrm{C}$ NMR $\left(100 \mathrm{MHz}, \mathrm{CDCl}_{3}\right) \delta 168.0,167.9,137.9,137.5,135.4,135.4,128.6,128.5,128.4,128.4$, 128.3, 128.3, 118.0, 115.0, 67.1, 67.0, 56.9, 43.7, 31.4, 31.1 ppm. FT-IR (KBr): 3068, 3034, 2976, 2936, 2919, 2849, 1753, 1734, 1640, 1498, 1377, 1265, 1215, 1144, 999, 917, 750, 698 $\mathrm{cm}^{-1}$. HRMS $\left(\mathrm{ESI}^{+}\right): \mathrm{m} / z$ calcd for $\mathrm{C}_{24} \mathrm{H}_{26} \mathrm{O}_{4} \mathrm{Na}[\mathrm{M}+\mathrm{Na}]^{+} 401.1723$, found $401.1716 .[\alpha]^{25.1}{ }_{\mathrm{D}}=$ $3.71\left(c=0.63, \mathrm{CHCl}_{3}\right)$. HPLC: Daicel Chiralpak IC, $n$-hexane $/ i-\mathrm{PrOH}=99 / 1,0.6 \mathrm{~mL} / \mathrm{min}$, $25^{\circ} \mathrm{C}, 210 \mathrm{~nm} ; 95: 5 \operatorname{er}\left[\mathrm{t}_{1}\right.$ (minor) $=19.013 \mathrm{~min}, \mathrm{t}_{2}$ (major) $\left.=19.823 \mathrm{~min}\right]$.

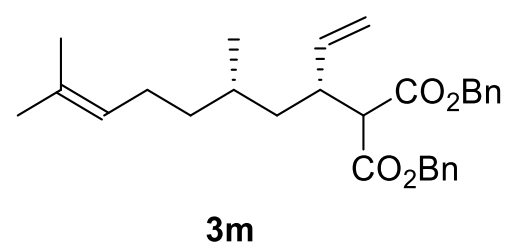

The compound was prepared according to the general procedure $\left(50{ }^{\circ} \mathrm{C}, 40 \mathrm{hr}\right)$. The crude reaction mixture was purified on silica gel (petroleum ether/ethyl acetate 20/1) to afford $\mathbf{3 m}$ as a colorless oil (40.0 mg, 89\%).

$\mathbf{R}_{f}=0.60$ (petroleum ether/ethyl acetate 10/1). ${ }^{1} \mathbf{H}$ NMR $\left(400 \mathrm{MHz}, \mathrm{CDCl}_{3}\right) \delta \delta$ 7.33-7.29 (m, $10 \mathrm{H}), 5.62(\mathrm{ddd}, \mathrm{J}=8.3,9.7,16.9 \mathrm{~Hz}, 1 \mathrm{H}), 5.14(\mathrm{~s}, 2 \mathrm{H}), 5.10(\mathrm{~s}, 2 \mathrm{H}), 5.05(\mathrm{~m}, 1 \mathrm{H}), 5.00(\mathrm{dd}$, $\mathrm{J}=1.9,8.3 \mathrm{~Hz}, 2 \mathrm{H}), 3.43(\mathrm{~d}, \mathrm{~J}=8.5 \mathrm{~Hz}, 1 \mathrm{H}), 2.91(\mathrm{tdd}, \mathrm{J}=4.4,8.5,9.7 \mathrm{~Hz}, 1 \mathrm{H}), 1.96(\mathrm{~m}, 1 \mathrm{H})$, $1.84(\mathrm{~m}, 1 \mathrm{H}), 1.67(\mathrm{~s}, 3 \mathrm{H}), 1.58(\mathrm{~s}, 3 \mathrm{H}), 1.45-1.35(\mathrm{~m}, 2 \mathrm{H}), 1.33-1.12(\mathrm{~m}, 2 \mathrm{H}), 0.95(\mathrm{ddt}, \mathrm{J}=$ 4.4, 8.5, $13.8 \mathrm{~Hz}, 1 \mathrm{H}), 0.82(\mathrm{~d}, \mathrm{~J}=6.5 \mathrm{~Hz}, 3 \mathrm{H}) .{ }^{13} \mathbf{C} \mathbf{~ N M R}\left(100 \mathrm{MHz}, \mathrm{CDCl}_{3}\right) \delta 168.1,167.9$, 138.2, 135.4, 135.4, 131.1, 128.5, 128.5, 128.4, 128.4, 128.3, 128.3, 124.9, 117.4, 67.1, 66.9, 57.2, 42.1, 39.9, 35.1, 29.6, 25.7, 25.1, 20.4, 17.6 ppm. FT-IR (KBr): 3095, 3066, 3034, 2961, 2914, 2875, 2851, 1754, 1735, 1641, 1498, 1377, 1335, 1261, 1231, 1215, 1139, 1000, 920 , 749, $697 \mathrm{~cm}^{-1}$. HRMS $\left(\mathrm{ESI}^{+}\right): \mathrm{m} / z$ calcd for $\mathrm{C}_{29} \mathrm{H}_{36} \mathrm{O}_{4} \mathrm{Na}[\mathrm{M}+\mathrm{Na}]^{+}$471.2506, found 471.2512. $[\boldsymbol{\alpha}]^{25.0}{ }_{\mathrm{D}}=-8.19\left(c=0.94, \mathrm{CHCl}_{3}\right)$. HPLC: Daicel Chiralpak AD-H, $n$-hexane/i-PrOH $=99 / 1$, $0.7 \mathrm{~mL} / \mathrm{min}, 25^{\circ} \mathrm{C}, 210 \mathrm{~nm} ; 7: 1 \mathrm{dr}\left[\mathrm{t}_{1}\right.$ (major) $=15.295 \mathrm{~min}, \mathrm{t}_{2}$ (minor) $=16.573 \mathrm{~min}$ ]. 


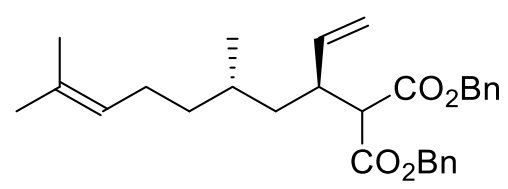

$3 n$

The compound was prepared according to the general procedure $\left(70{ }^{\circ} \mathrm{C}, 40 \mathrm{hr},(S)\right.$-Ir-TolBINAP). The crude reaction mixture was purified on silica gel (petroleum ether/ethyl acetate 20/1) to afford $\mathbf{3 n}$ as a colorless oil (40.6 $\mathrm{mg}, 91 \%)$.

$\mathbf{R}_{f}=0.60$ (petroleum ether/ethyl acetate 10/1). ${ }^{1} \mathbf{H}$ NMR $\left(400 \mathrm{MHz}, \mathrm{CDCl}_{3}\right) \delta$ 7.34-7.27 (m, $10 \mathrm{H}), 5.56$ (ddd, J = 8.5, 9.4, $15.5 \mathrm{~Hz}, 1 \mathrm{H}), 5.15(\mathrm{~d}, \mathrm{~J}=7.3 \mathrm{~Hz}, 2 \mathrm{H}), 5.10(\mathrm{~s}, 2 \mathrm{H}), 5.06-4.97$ (m, 3H), $3.42(\mathrm{~d}, \mathrm{~J}=8.8 \mathrm{~Hz}, 1 \mathrm{H}), 2.90(\mathrm{tdd}, \mathrm{J}=8.8,9.4,15.5 \mathrm{~Hz}, 1 \mathrm{H}), 1.93-1.86(\mathrm{~m}, 2 \mathrm{H}), 1.67$ (s, 3H), 1.58 (s, 3H), 1.39-1.34 (m, 2H), $1.14(\mathrm{ddd}, \mathrm{J}=6.5,8.7,15.5 \mathrm{~Hz}, 2 \mathrm{H}), 1.07(\mathrm{~m}, 1 \mathrm{H})$, $0.79(\mathrm{~d}, \mathrm{~J}=6.4 \mathrm{~Hz}, 3 \mathrm{H}) .{ }^{13} \mathrm{C}$ NMR $\left(100 \mathrm{MHz}, \mathrm{CDCl}_{3}\right) \delta 168.0,168.0,137.9,135.4,135.4,131.2$, 128.6, 128.5, 128.4, 128.4, 128.3, 128.3, 124.7, 117.6, 67.1, 67.0, 57.6, 42.2, 39.3, 38.0, 29.6, 25.7, 25.4, 18.4, 17.7 ppm. FT-IR (KBr): 3095, 3066, 3034, 2961, 2914, 2875, 2851, 1754, 1735, 1641, 1498, 1377, 1335, 1261, 1231, 1215, 1139, 1000, 920, 749, $697 \mathrm{~cm}^{-1}$. HRMS $\left(\mathrm{ESI}^{+}\right): \mathrm{m} / z$ calcd for $\mathrm{C}_{29} \mathrm{H}_{36} \mathrm{O}_{4} \mathrm{Na}[\mathrm{M}+\mathrm{Na}]^{+} 471.2506$, found $471.2512 .[\alpha]^{25.3} \mathrm{D}=6.67(c=0.54$, $\mathrm{CHCl}_{3}$ ). HPLC: Daicel Chiralpak AD-H, $n$-hexane $i-\mathrm{PrOH}=99 / 1,0.7 \mathrm{~mL} / \mathrm{min}, 25^{\circ} \mathrm{C}, 210 \mathrm{~nm}$; $19: 1 \mathrm{dr}\left[\mathrm{t}_{1}(\right.$ minor $)=15.553 \mathrm{~min}, \mathrm{t}_{2}($ major $\left.)=16.465 \mathrm{~min}\right]$.

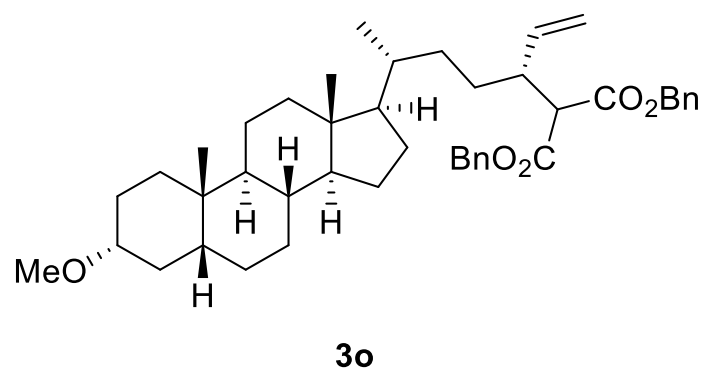

The compound was prepared according to the general procedure $\left(50{ }^{\circ} \mathrm{C}, 40 \mathrm{hr}\right)$. The crude reaction mixture was purified on silica gel (petroleum ether/ethyl acetate 20/1) to afford 3o as a colorless oil $(56.9 \mathrm{mg}, 85 \%)$.

$\mathbf{R}_{f}=0.43$ (petroleum ether/ethyl acetate 10/1). ${ }^{1} \mathbf{H}$ NMR $\left(400 \mathrm{MHz}, \mathrm{CDCl}_{3}\right) \delta 7.32(\mathrm{~m}, 10 \mathrm{H})$, 5.65 (ddd, J = 8.3, 9.5, 13.7 Hz, 1H), 5.15 (s, 2H), 5.11 (s, 2H), 5.00 (dd, J = 2.2, $13.7 \mathrm{~Hz}, 2 \mathrm{H}$ ), $3.50(\mathrm{~d}, \mathrm{~J}=8.4 \mathrm{~Hz}, 1 \mathrm{H}), 3.35(\mathrm{~s}, 3 \mathrm{H}), 3.16(\mathrm{dt}, \mathrm{J}=8.3,9.1 \mathrm{~Hz}, 1 \mathrm{H}), 2.71(\mathrm{tdd}, \mathrm{J}=8.3,9.1$, 13.3Hz, 1H), $1.92(\mathrm{~d}, \mathrm{~J}=12.2 \mathrm{~Hz}, 1 \mathrm{H}), 1.85(\mathrm{~m}, 1 \mathrm{H}), 1.82-1.65(\mathrm{~m}, 4 \mathrm{H}), 1.63-1.48(\mathrm{~m}, 4 \mathrm{H})$, 1.41-1.35 (m, 6H), $1.31-1.20(\mathrm{~m}, 3 \mathrm{H}), 1.21-1.04$ (m, 5H), 1.02-0.98 (m, 4H), $0.92(\mathrm{~s}, 3 \mathrm{H})$, $0.81(\mathrm{~d}, \mathrm{~J}=6.5 \mathrm{~Hz}, 3 \mathrm{H}), 0.60(\mathrm{~s}, 3 \mathrm{H}) .{ }^{13} \mathbf{C} \mathbf{~ N M R}\left(100 \mathrm{MHz}, \mathrm{CDCl}_{3}\right) \delta 168.2,168.0,138.3,135.4$, 
135.4, 128.6, 128.5, 128.4, 128.3, 128.3, 128.3, 117.4, 80.5, 67.1, 66.9, 56.8, 56.4, 56.0, 55.6, $44.8,42.7,42.1,40.4,40.2,35.9,35.9,35.3,34.9,33.2,32.8,28.9,28.2,27.4,26.8,26.4,24.2$, 23.5, 20.8, 18.8, 12.0 ppm. FT-IR (KBr): 3066, 3033, 2934, 2886, 2864, 2820, 1754, 1735 , 1641, 1498, 1454, 1375, 1311, 1261, 1235, 1215, 1173, 1148, 1099,996, 917, 749, $697 \mathrm{~cm}^{-1}$. HRMS $\left(\mathrm{ESI}^{+}\right): \mathrm{m} / z$ calcd for $\mathrm{C}_{44} \mathrm{H}_{60} \mathrm{O}_{5} \mathrm{Na}[\mathrm{M}+\mathrm{Na}]^{+}$691.4333, found 691.4327. $[\alpha]^{25.7} \mathrm{D}=23.5$ $\left(c=1.04, \mathrm{CHCl}_{3}\right)$. HPLC: Daicel Chiralpak AD-H, $n$-hexane $/ i-\mathrm{PrOH}=97 / 3,1.0 \mathrm{~mL} / \mathrm{min}$, $25^{\circ} \mathrm{C}, 210 \mathrm{~nm} ; 49: 1 \mathrm{dr}\left[\mathrm{t}_{1}\right.$ (minor) $=14.606 \mathrm{~min}, \mathrm{t}_{2}$ (major) $\left.=15.616 \mathrm{~min}\right]$.

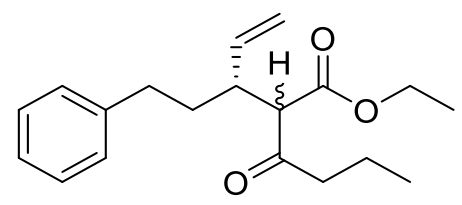

$3 p$

The compound was prepared according to the general procedure $\left(50^{\circ} \mathrm{C}, 40 \mathrm{hr}\right)$. The crude reaction mixture was purified on silica gel (toluene) to afford $\mathbf{3 p}$ as a colorless oil mixture of diastereoisomers $(\mathrm{dr}=1: 1$, determined by HPLC) $(29.1 \mathrm{mg}, 96 \%)$.

$\mathbf{R}_{f}=0.47$ (petroleum ether/ethyl acetate 15/1). ${ }^{1} \mathbf{H}$ NMR $\left(400 \mathrm{MHz}, \mathrm{CDCl}_{3}\right) \delta 7.38-7.27(\mathrm{~m}$, $2 \mathrm{H}), 7.28-7.15(\mathrm{~m}, 3 \mathrm{H}), 5.73(\mathrm{~m}, 1 \mathrm{H}), 5.23-5.20(\mathrm{~m}, 2 \mathrm{H}) ., 4.29-4.17(\mathrm{~m}, 2 \mathrm{H}), 3.54(\mathrm{~m}, 1 \mathrm{H})$, $2.95(\mathrm{~m}, 1 \mathrm{H}), 2.74(\mathrm{~m}, 1 \mathrm{H}), 2.57(\mathrm{~m}, 1 \mathrm{H}), 2.53-2.40(\mathrm{~m}, 2 \mathrm{H}), 1.85(\mathrm{~m}, 1 \mathrm{H}), 1.75(\mathrm{~m}, 1 \mathrm{H})$, $1.67-1.57(\mathrm{~m}, 2 \mathrm{H}), 1.30(\mathrm{t}, \mathrm{J}=7.1 \mathrm{~Hz}, 3 \mathrm{H}), 0.94(\mathrm{t}, \mathrm{J}=7.4 \mathrm{~Hz}, 3 \mathrm{H}) .{ }^{13} \mathbf{C ~ N M R}\left(100 \mathrm{MHz}, \mathrm{CDCl}_{3}\right)$ $\delta$ 204.3, 204.3, 168.6, 168.4, 141.9, 141.8, 137.9, 137.9, 128.4, 128.4, 128.4, 128.3, 125.9, $125.9,118.2,118.0,64.4,64.3,61.3,61.2,44.7,44.3,43.7,43.5,34.3,34.2,33.3,33.2,16.8$, 16.6, 14.1, 14.1, 13.6, 13.5 ppm. FT-IR (KBr): 3083, 3064, 3026, 2963, 2934, 2875, 1743 , 1713, 1496, 1455, 1367, 1299, 1263, 1237, 1152, 1117, 1095, 1029, 1000, 921, 749, $700 \mathrm{~cm}^{-1}$. HRMS $\left(\mathrm{ESI}^{+}\right): \mathrm{m} / z$ calcd for $\mathrm{C}_{19} \mathrm{H}_{26} \mathrm{O}_{3} \mathrm{Na}[\mathrm{M}+\mathrm{Na}]^{+} 325.1774$, found 325.1779. HPLC: Daicel Chiralpak AD-H, $n$-hexane $/ i$-PrOH $=99 / 1,1.0 \mathrm{~mL} / \mathrm{min}, 25^{\circ} \mathrm{C}, 210 \mathrm{~nm} ; 96: 4 \operatorname{er}\left[\mathrm{t}_{1}\right.$ (major) $=7.56$ min, $\left.\mathrm{t}_{2}(\operatorname{minor})=24.802 \mathrm{~min}\right]$ and $96: 4 \operatorname{er}\left[\mathrm{t}_{1}(\right.$ minor $)=9.039 \mathrm{~min}, \mathrm{t}_{2}($ major $)=13.869$ $\min ]$.

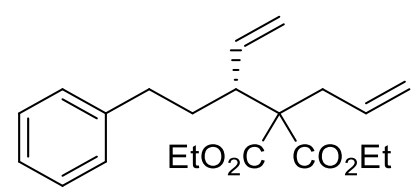

$3 q$ 
The compound was prepared according to the general procedure $\left(50{ }^{\circ} \mathrm{C}, 40 \mathrm{hr}\right)$. The crude reaction mixture was purified on silica gel (toluene) to afford $\mathbf{3 q}$ as a colorless oil (31.3mg, $91 \%)$.

$\mathbf{R}_{f}=0.50$ (petroleum ether/ethyl acetate 15/1). ${ }^{1} \mathbf{H}$ NMR $\left(400 \mathrm{MHz}, \mathrm{CDCl}_{3}\right) \delta 7.41-7.22(\mathrm{~m}$, 2H), $7.21-7.03(\mathrm{~m}, 3 \mathrm{H}), 5.76(\mathrm{~m}, 1 \mathrm{H}), 5.64(\mathrm{ddd}, J=9.3,10.1,17.0 \mathrm{~Hz}, 1 \mathrm{H}), 5.22(\mathrm{dd}, J=$ 2.0, $10.2 \mathrm{~Hz}, 1 \mathrm{H}$ ), 5.13 (dd, $J=2.0,17.0 \mathrm{~Hz}, 1 \mathrm{H}), 5.09-4.99$ (m, 2H), 4.16 (ddd, $J=2.2,5.4$, $7.3 \mathrm{~Hz}, 4 \mathrm{H}), 2.72-2.65(\mathrm{~m}, 2 \mathrm{H}), 2.62(\mathrm{~d}, \mathrm{~J}=7.4 \mathrm{~Hz}, 2 \mathrm{H}), 2.47$ (ddd, $J=6.7,10.3,13.8 \mathrm{~Hz}, 1 \mathrm{H})$, $2.0(\mathrm{~m}, 1 \mathrm{H}), 1.50(\mathrm{~m}, 1 \mathrm{H}), 1.23(\mathrm{t}, J=7.2 \mathrm{~Hz}, 6 \mathrm{H}) .{ }^{13} \mathbf{C ~ N M R}\left(100 \mathrm{MHz}, \mathrm{CDCl}_{3}\right) \delta 170.5,170.3$, $142.2,136.9,133.4,128.5,128.4,128.3,128.3,125.7,119.1,119.0,118.4,118.3,61.1,61.0$, 48.4, 38.9, 34.2, 32.3, 14.1 ppm. FT-IR (KBr): 3078, 3026, 2980, 2960, 2932, 2862, 1727, 1639, 1603, 1484, 1454, 1445, 1367, 1229, 1280, 1222, 1197, 1140, 1096, 1044, 1030, 1000, 920, 750, $700 \mathrm{~cm}^{-1}$. HRMS $\left(\mathrm{ESI}^{+}\right): \mathrm{m} / z$ calcd for $\mathrm{C}_{21} \mathrm{H}_{28} \mathrm{O}_{4} \mathrm{Na}[\mathrm{M}+\mathrm{Na}]^{+}$367.1880, found 367.1887. $[\alpha]^{19.9}{ }_{\mathrm{D}}=3.42\left(c=0.24, \mathrm{CHCl}_{3}\right)$. HPLC: Daicel Chiralpak IC, $n$-hexane/ $i-\operatorname{PrOH}=$ 99/1, $1.0 \mathrm{~mL} / \mathrm{min}, 25^{\circ} \mathrm{C}, 210 \mathrm{~nm} ; 69: 31$ er $\left[\mathrm{t}_{1}\right.$ (minor) $=6.321 \mathrm{~min}, \mathrm{t}_{2}$ (major) $\left.=7.073 \mathrm{~min}\right]$. 


\section{References}

[1] Kim, S. W.; Schempp, T. T.; Zbieg, J. R.; Stivala, C. E.; Krische, M. J. Angew. Chem. Int. Ed. 2019, 58, 7762 .

[2] Betz, P.; Krueger, A. ChemPhysChem 2012, 13, 2578.

[3] Han, S. B.; Han, H.; Krische, M. J. J. Am. Chem. Soc. 2010, 132, 1760. 
NMR Data

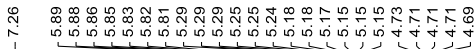

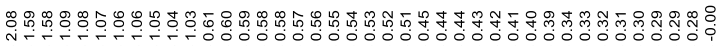<smiles>C=CC(OC(C)=O)C1CC1</smiles>

1e

${ }^{1} \mathrm{H}-\mathrm{NMR} 400 \mathrm{MHz} \mathrm{CDCl}_{3}$
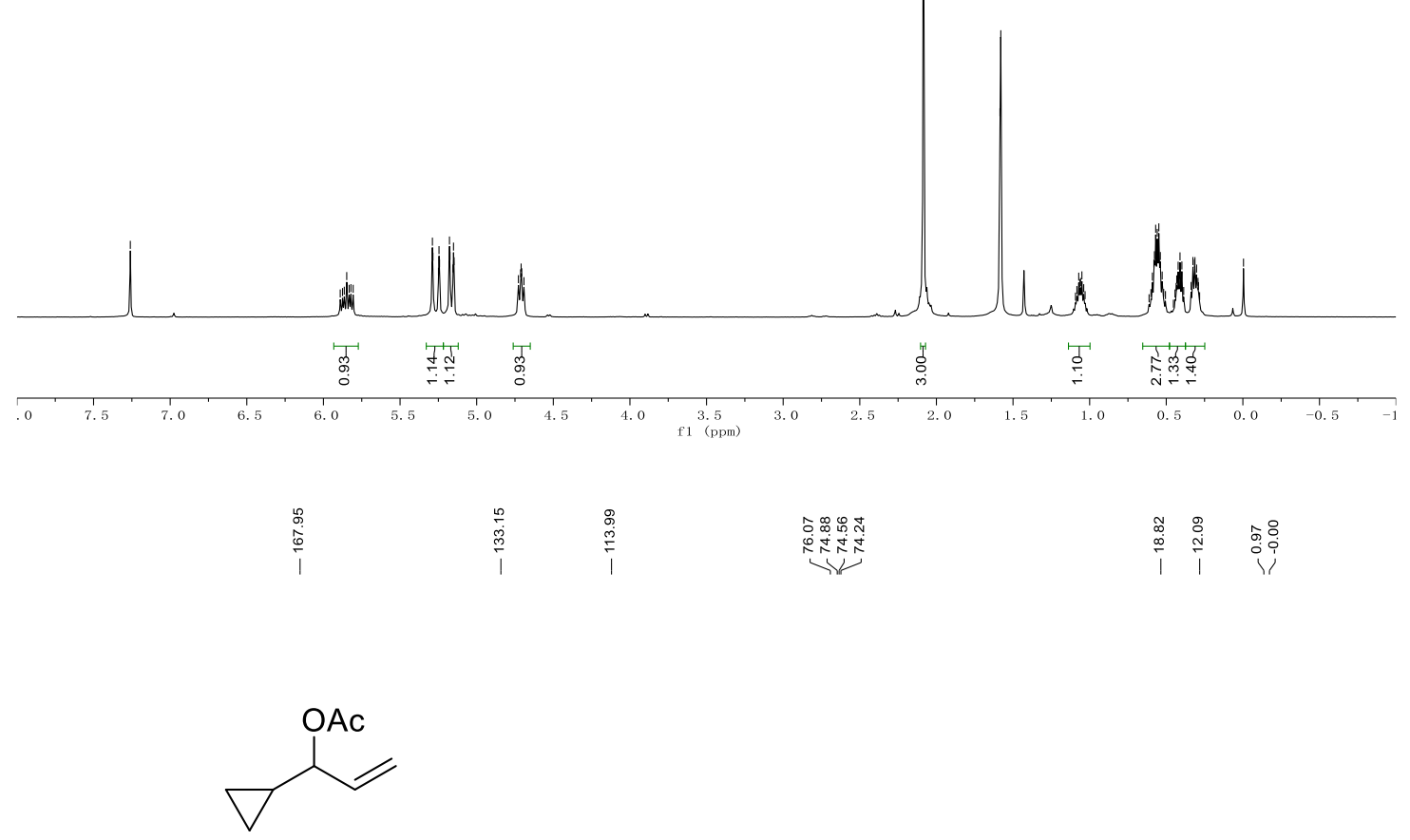

$1 \mathrm{e}$

${ }^{13} \mathrm{C}-\mathrm{NMR} 100 \mathrm{MHz} \mathrm{CDCl}_{3}$

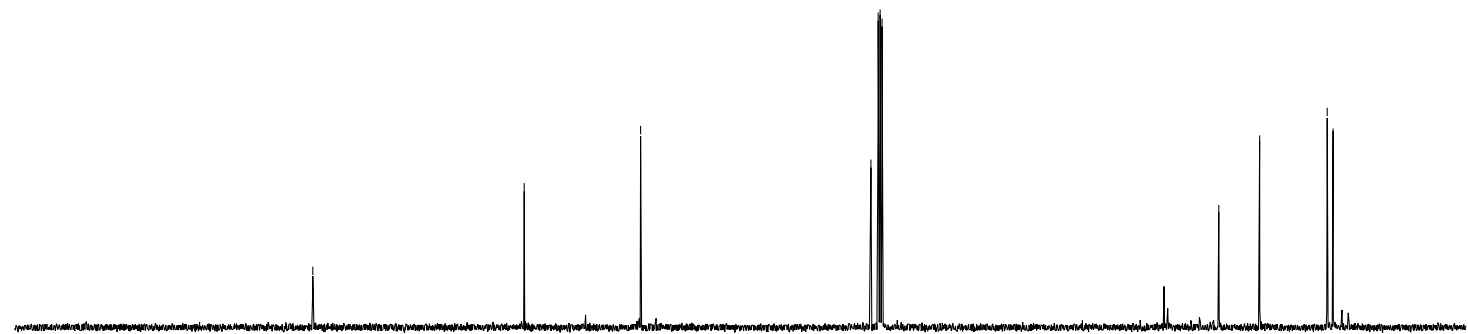

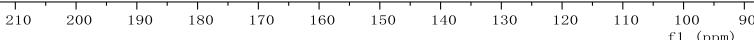



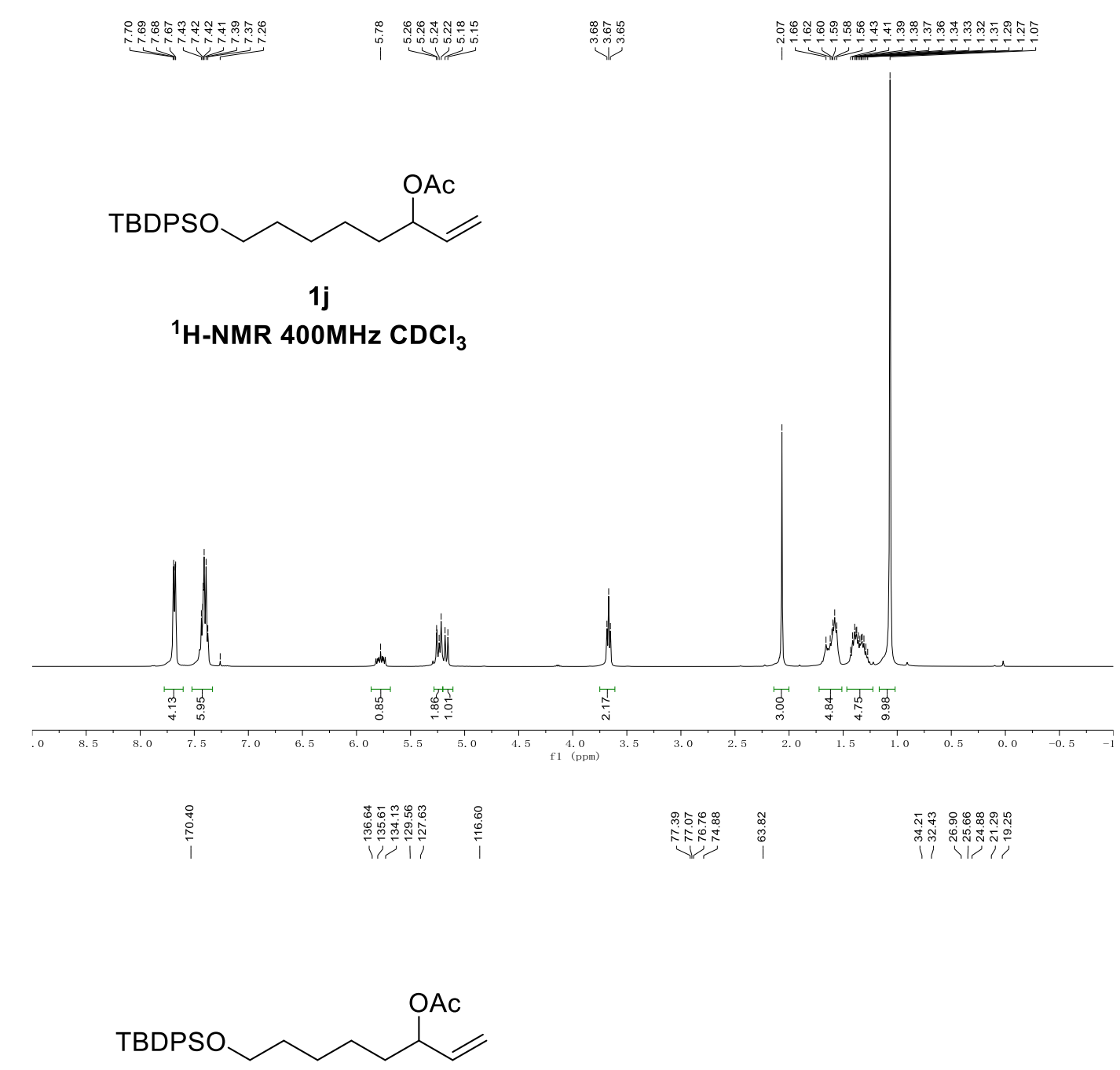

$1 \mathrm{j}$

${ }^{13} \mathrm{C}$-NMR $100 \mathrm{MHz}^{\mathrm{CDCl}}{ }_{3}$

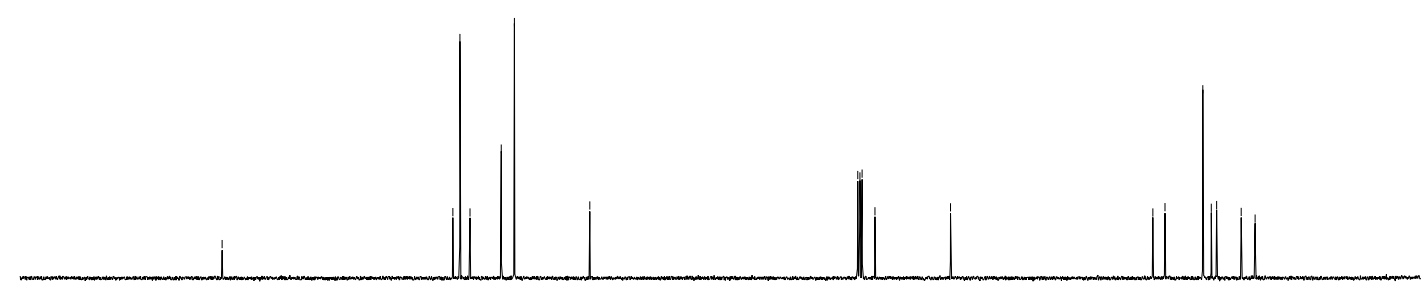

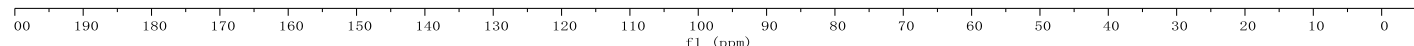



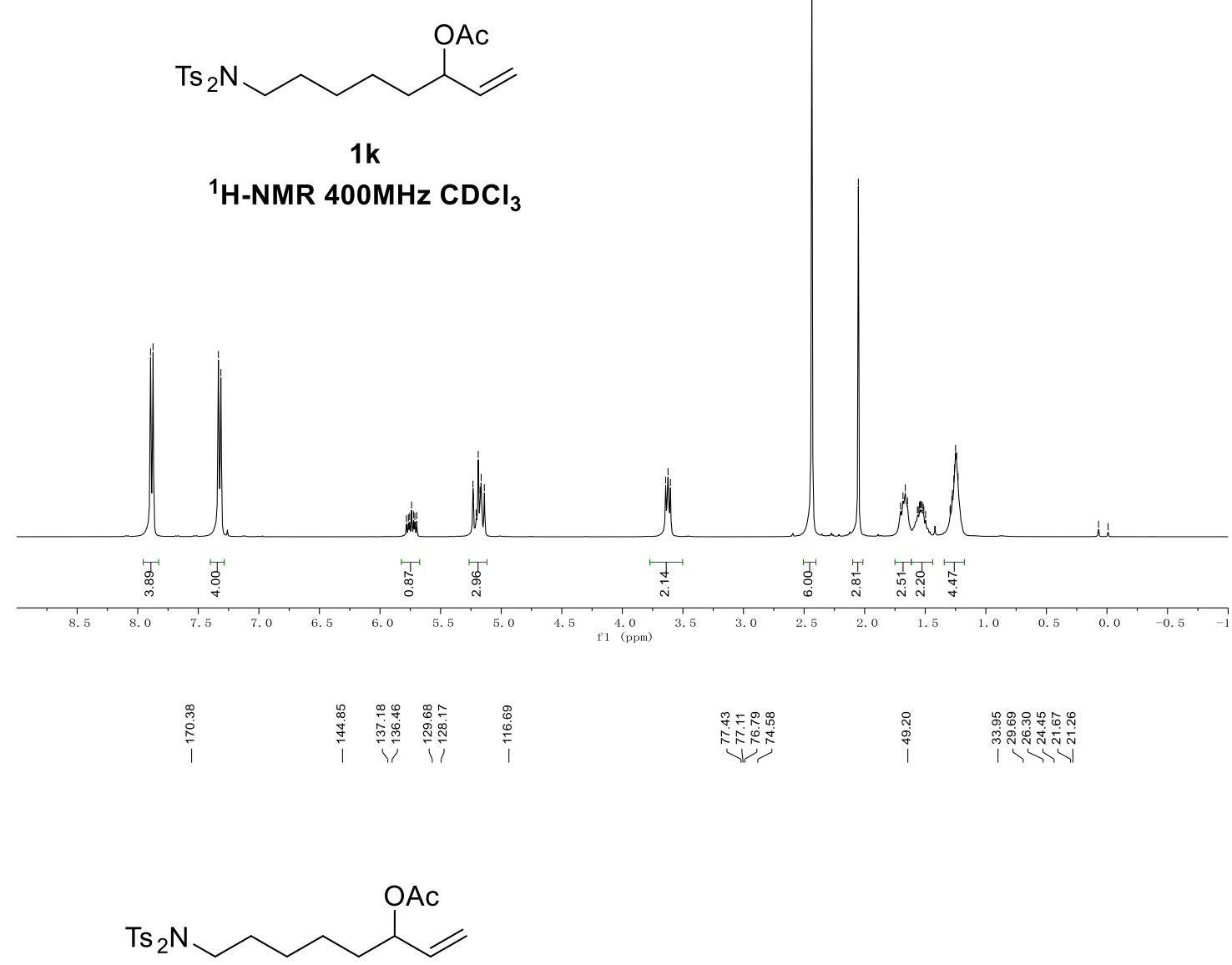

$1 \mathrm{k}$

${ }^{13} \mathrm{C}-\mathrm{NMR} 100 \mathrm{MHz} \mathrm{CDCl}_{3}$

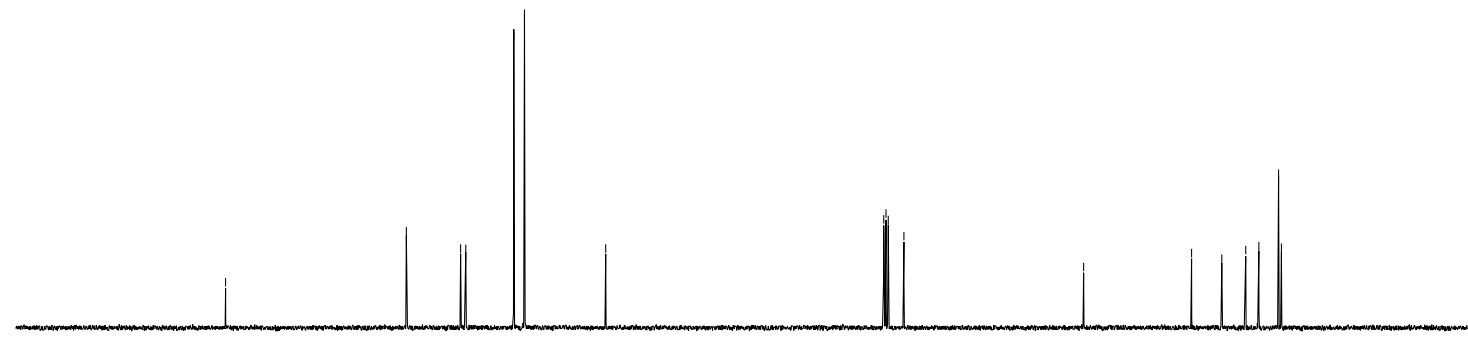

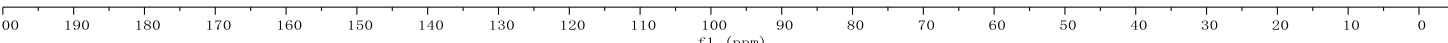



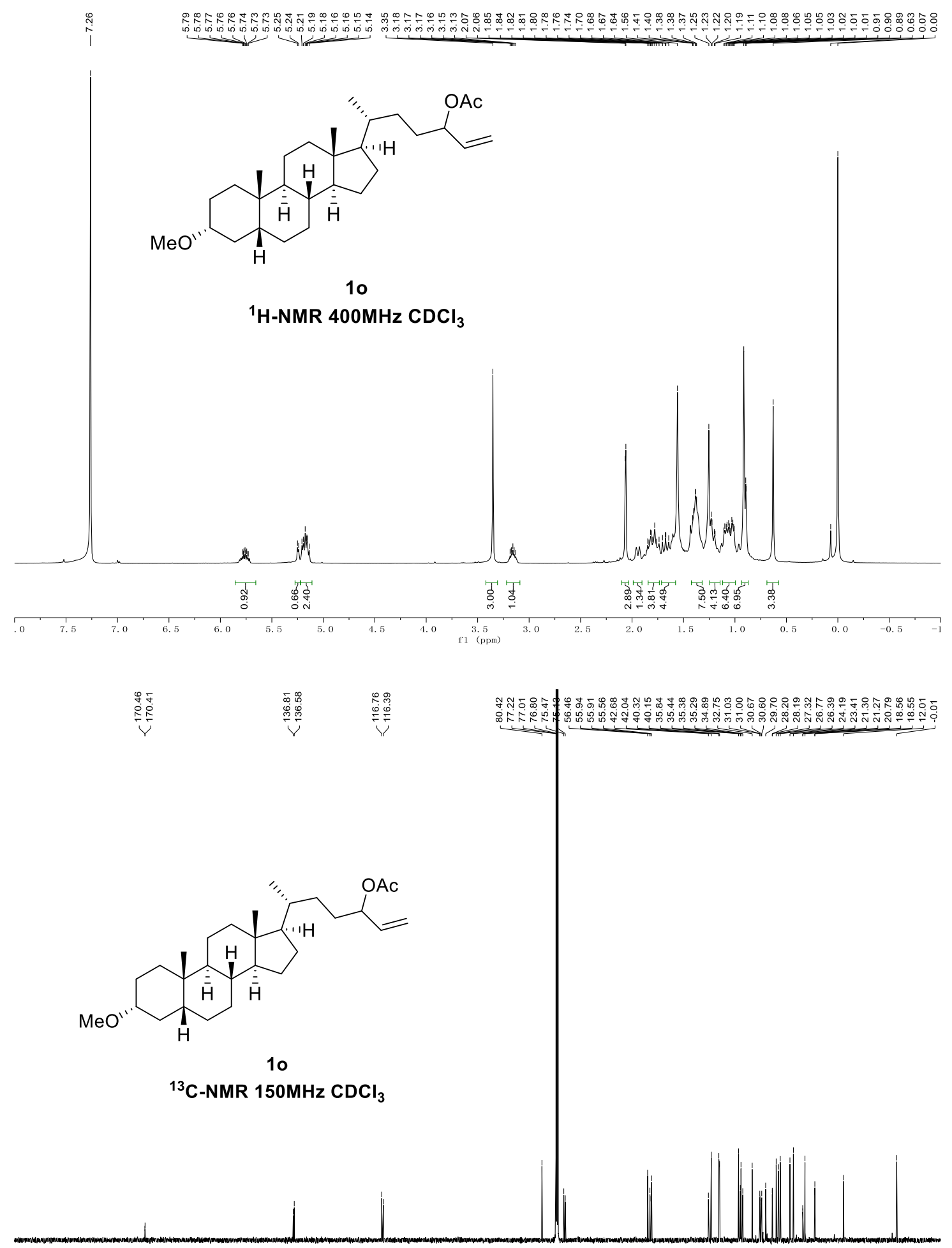

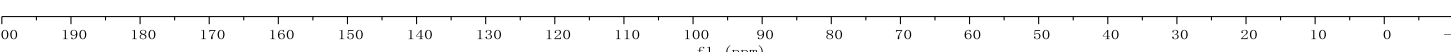




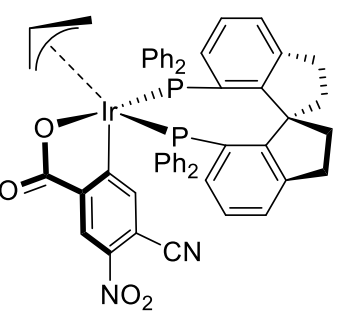

(R)-Ir-SDP

cat-2

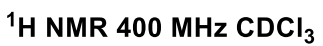

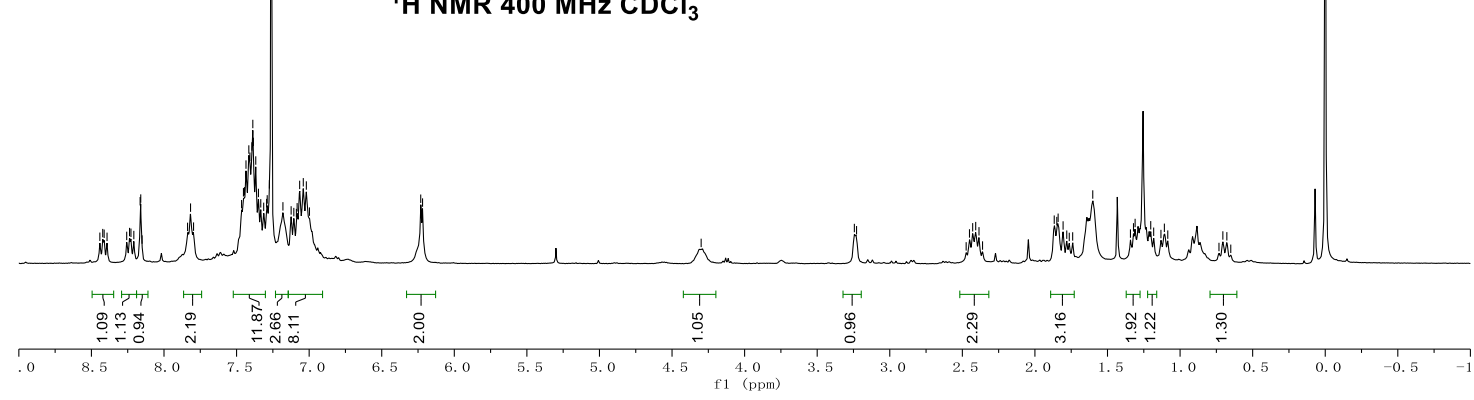

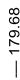

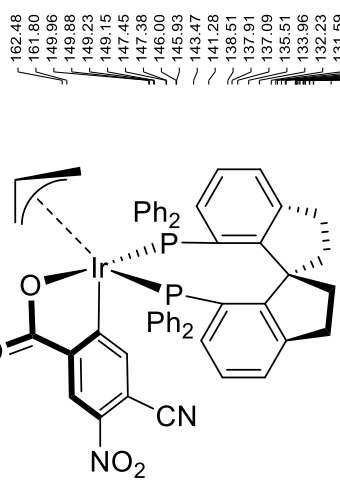

$(R)$-Ir-SDP

cat-2

${ }^{13} \mathrm{C}$ NMR $151 \mathrm{MHz} \mathrm{CDCl}_{3}$ 


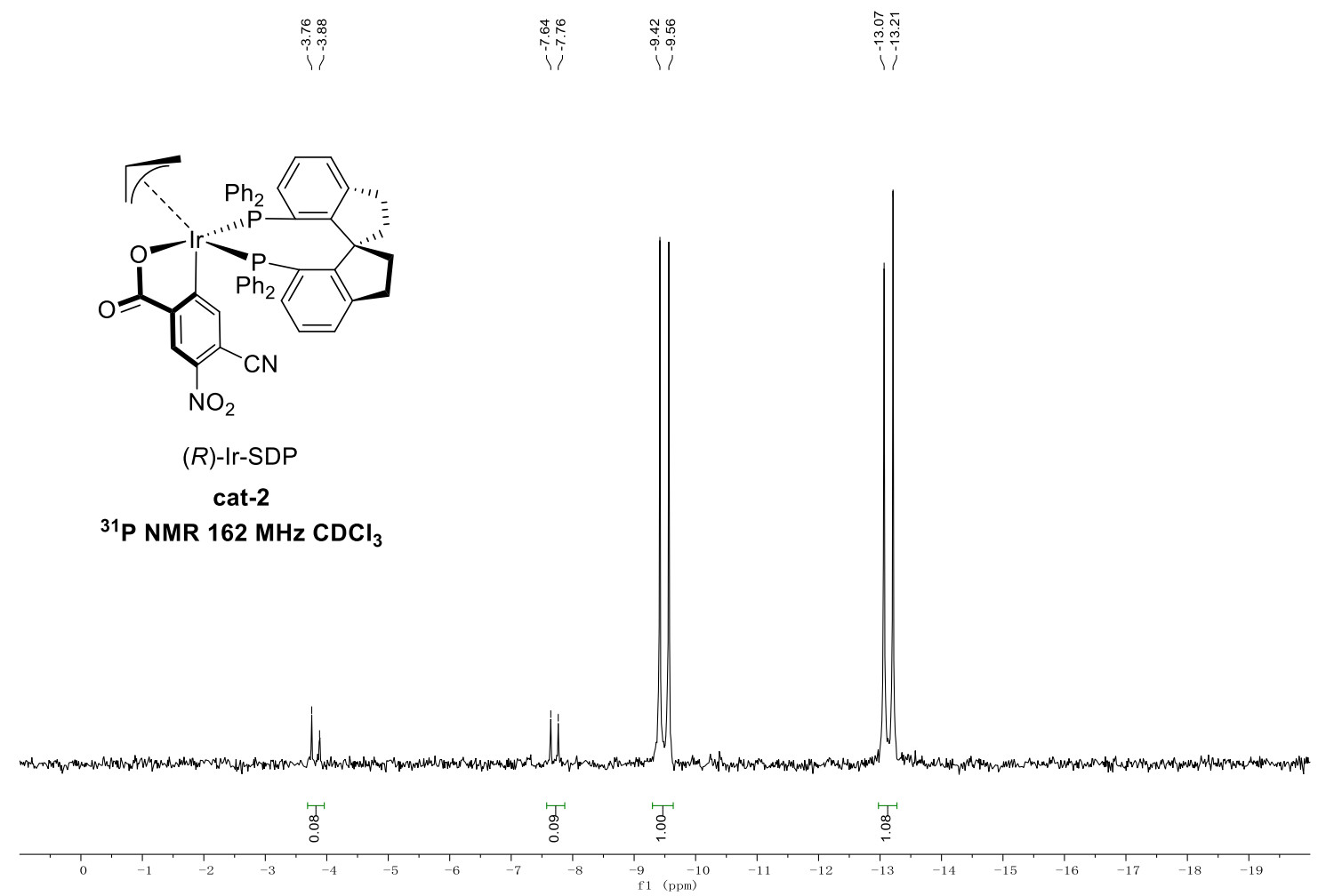




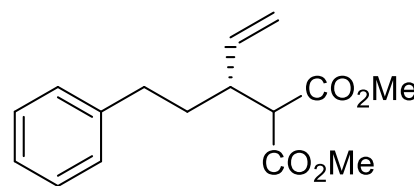

3a

${ }^{1} \mathrm{H}-\mathrm{NMR} \quad 400 \mathrm{MHz} \mathrm{CDCl}_{3}$

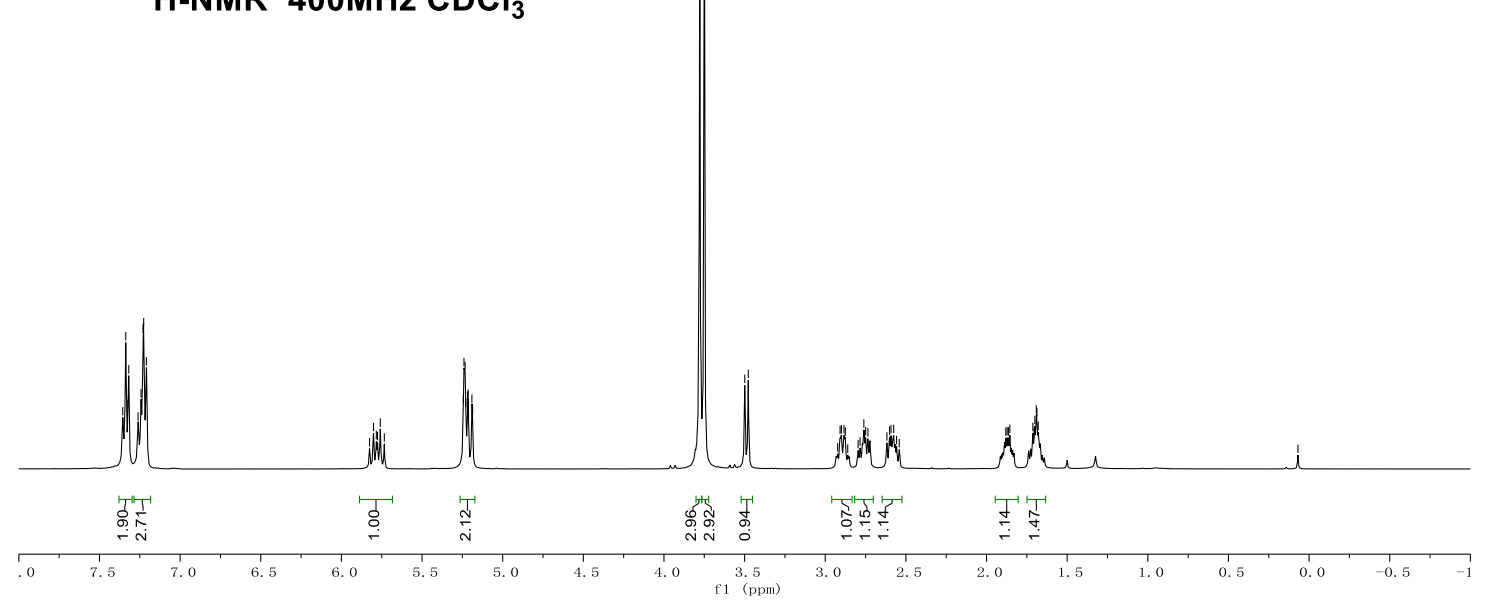

要

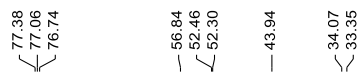

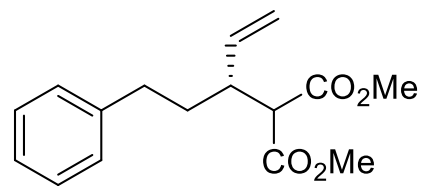

$3 a$

${ }^{13} \mathrm{C}$-NMR $100 \mathrm{MHz} \mathrm{CDCl}_{3}$

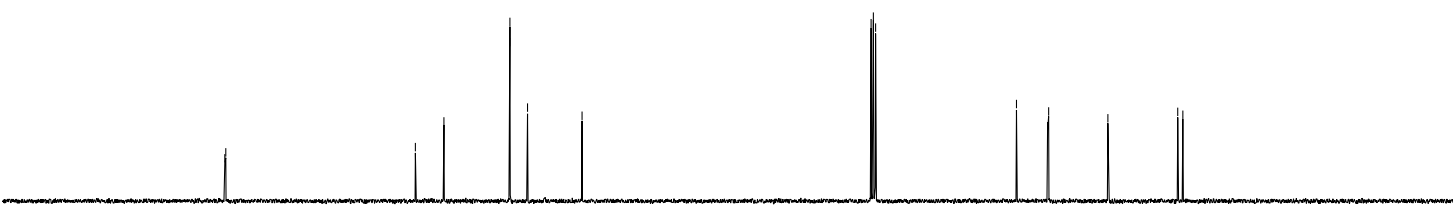

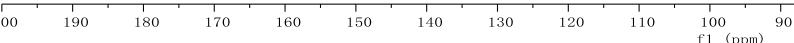


<smiles>C=C[C@@H](Cc1ccccc1)C(C(C)=O)C(C)=O</smiles>

$3 b$

${ }^{1} \mathrm{H}-\mathrm{NMR}$ 400MHz $\mathrm{CDCl}_{3}$
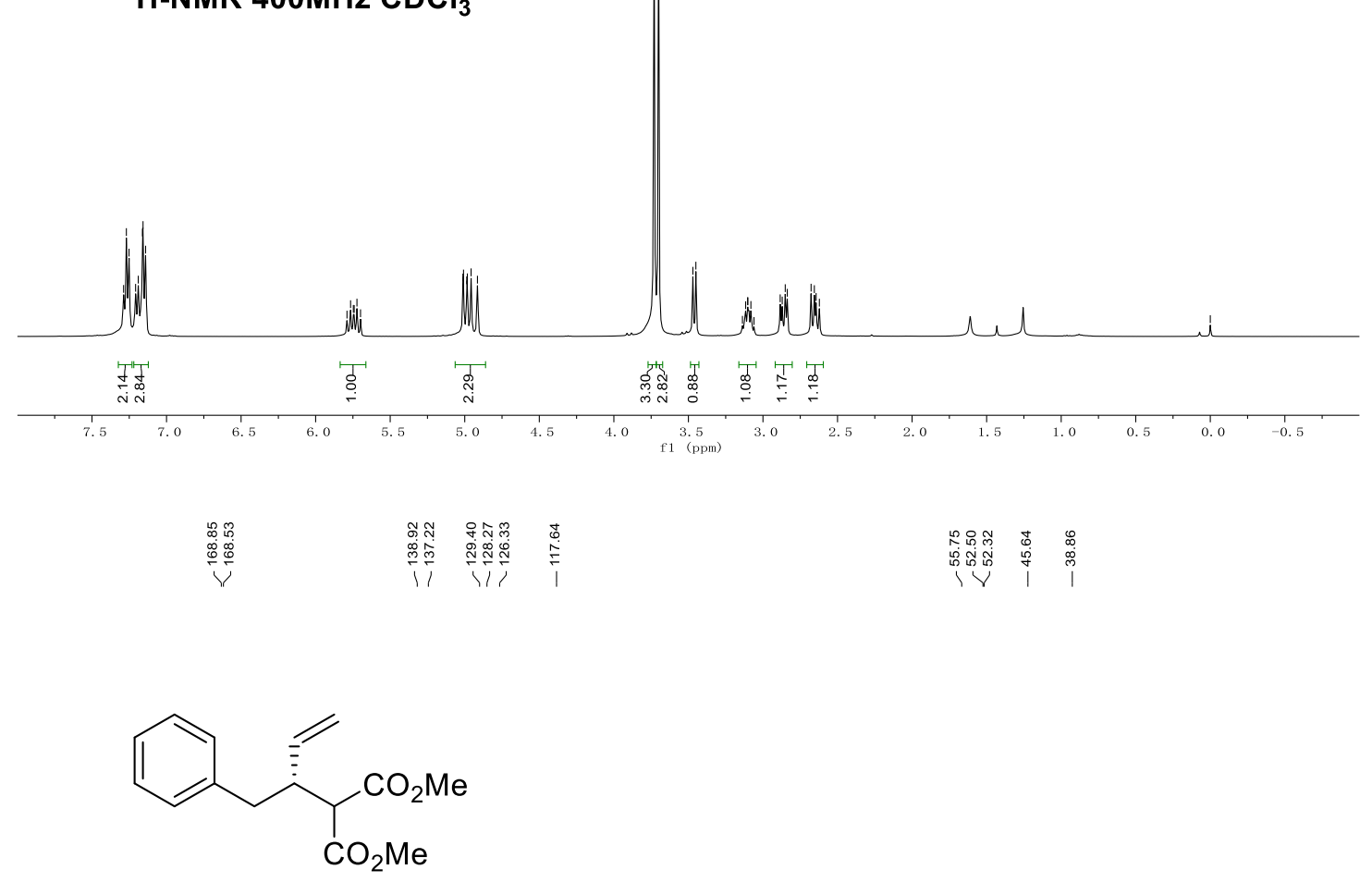

$3 b$

${ }^{13} \mathrm{C}$-NMR $100 \mathrm{MHz}^{\mathrm{CDCl}}{ }_{3}$

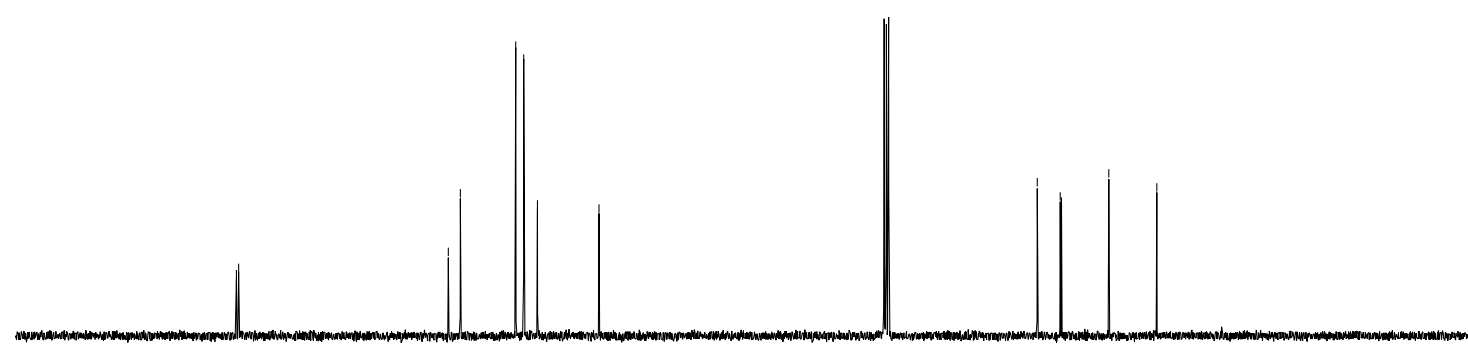


<smiles>C=C[C@H](C)C(C(=O)OCc1ccccc1)C(=O)OCc1ccccc1</smiles>

$3 c$

${ }^{1} \mathrm{H}-\mathrm{NMR} 400 \mathrm{MHz} \mathrm{CDCl}_{3}$

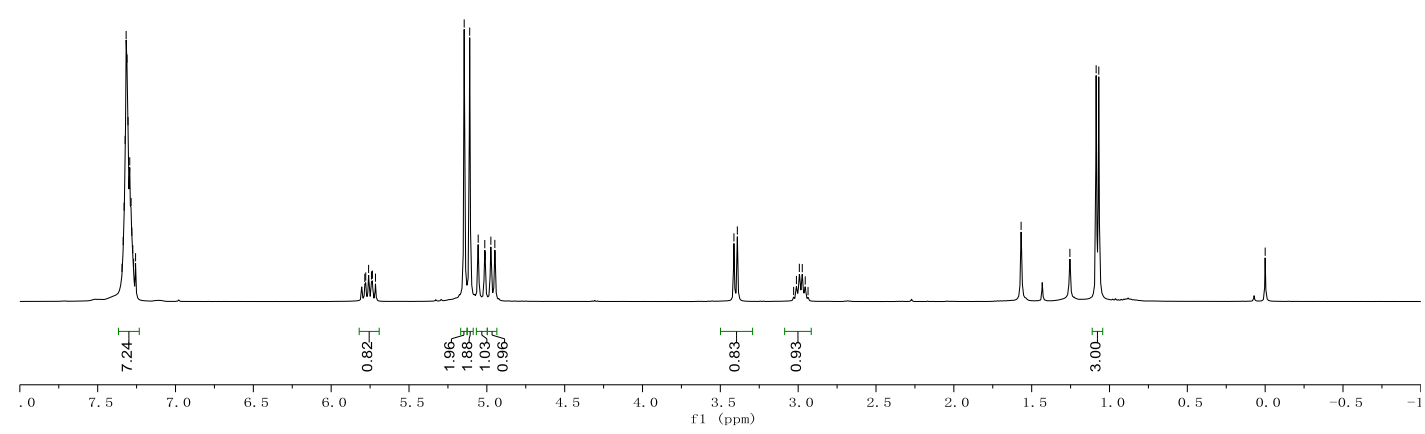

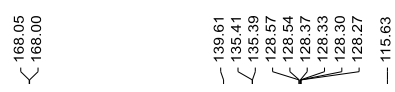<smiles>C=C[C@H](C)C(C(=O)OCc1ccccc1)C(=O)OCc1ccccc1</smiles>

$3 c$

${ }^{13} \mathrm{C}$-NMR $100 \mathrm{MHz} \mathrm{CDCl}_{3}$

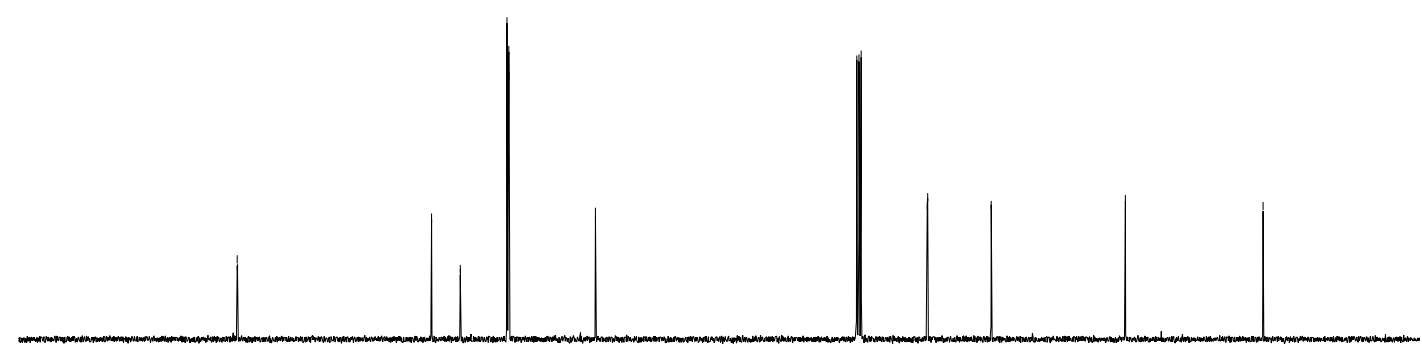

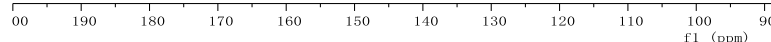


<smiles>C=C[C@H](CCC)C(C(=O)Br)C(=O)OBr</smiles>

3d

${ }^{1} \mathrm{H}-\mathrm{NMR}$ 400MHz $\mathrm{CDCl}_{3}$

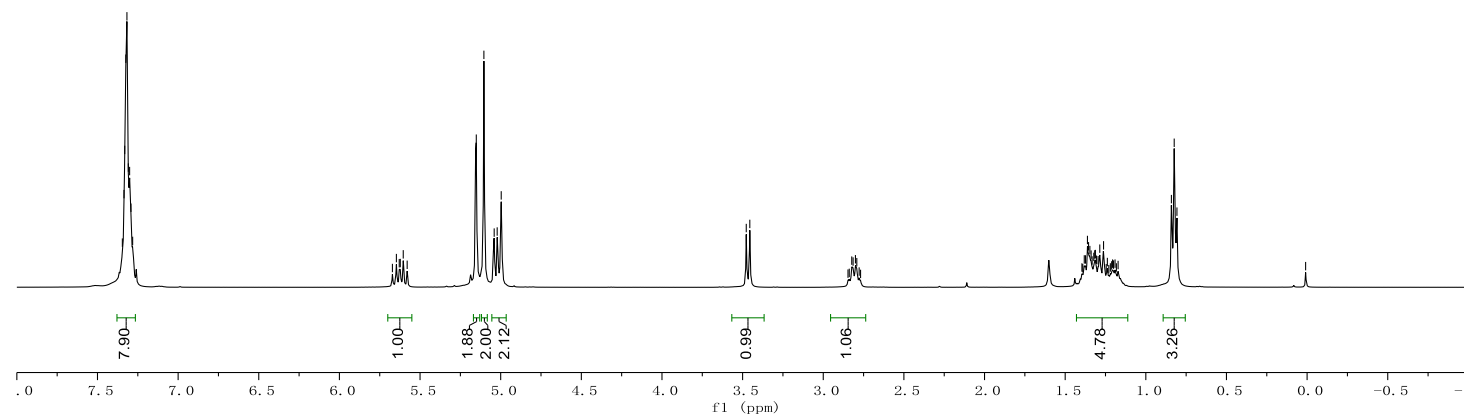

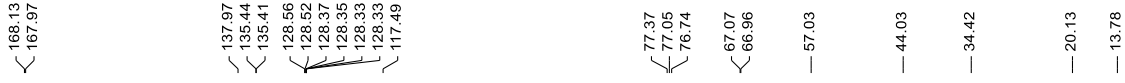<smiles>C=C[C@H](CCC)C(C(=O)OBr)C(=O)Oc1ccccc1</smiles>

$3 d$

${ }^{13} \mathrm{C}$-NMR $100 \mathrm{MHz}^{\mathrm{CDCl}}{ }_{3}$

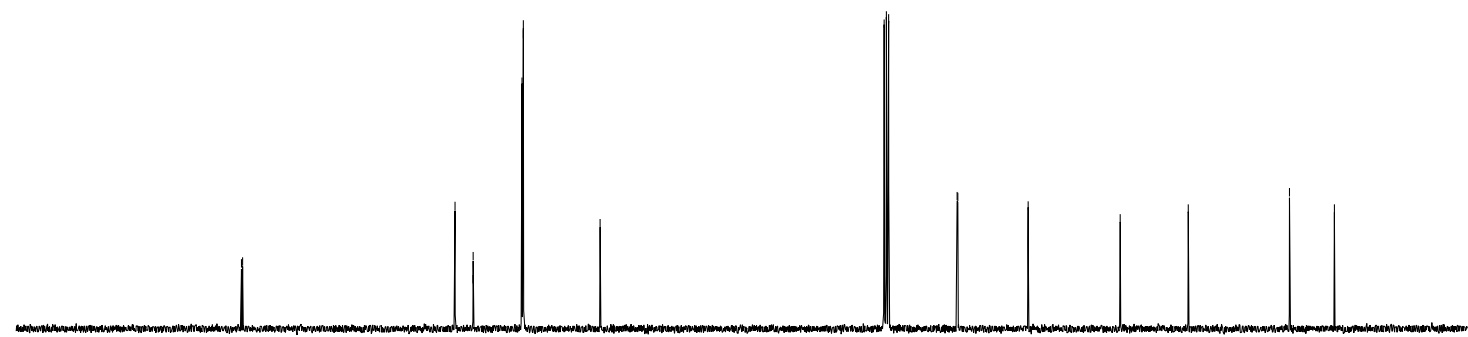

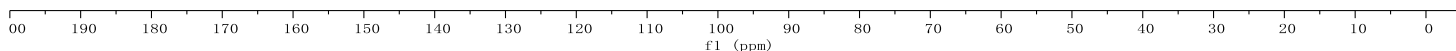




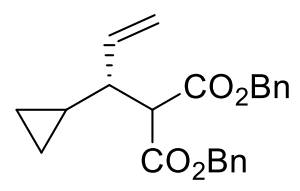

$3 e$
${ }^{1} \mathrm{H}-\mathrm{NMR}$
$400 \mathrm{MHz} \mathrm{CDCl}_{3}$
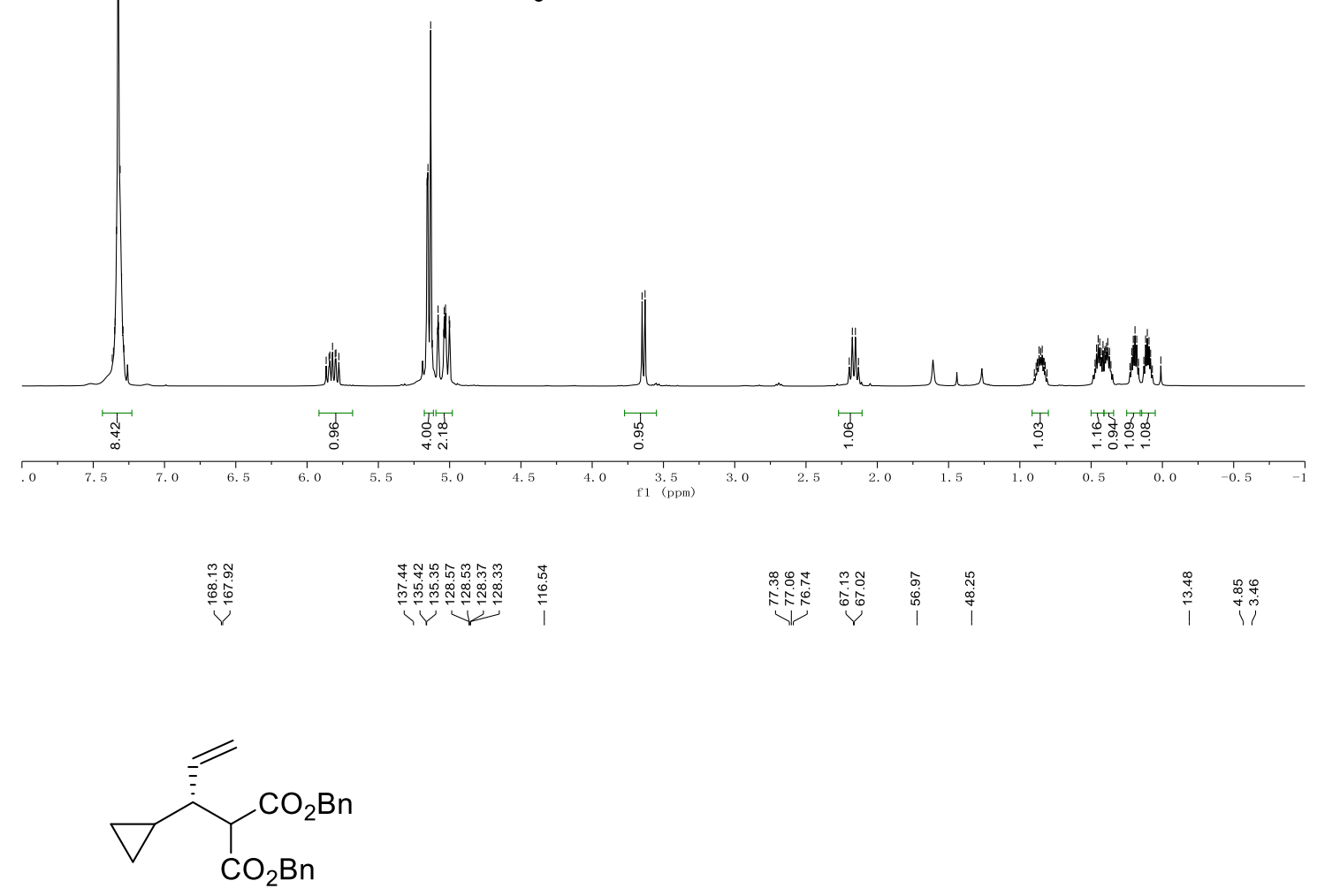

$3 e$

${ }^{13} \mathrm{C}-\mathrm{NMR} 100 \mathrm{MHz}^{\mathrm{CDCl}}{ }_{3}$

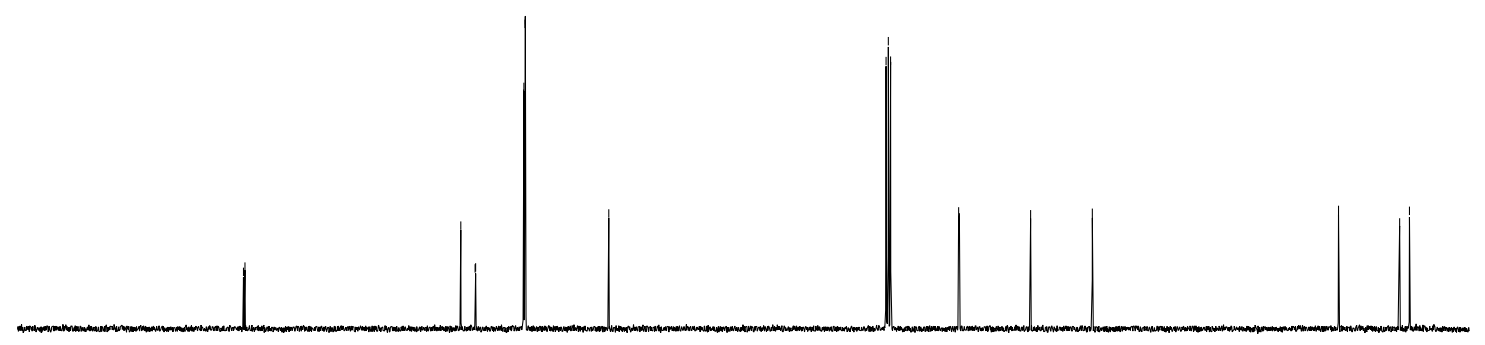

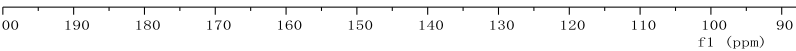




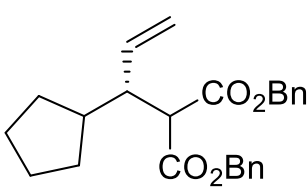

$3 f$

${ }^{1} \mathrm{H}-\mathrm{NMR}$ 400MHz $\mathrm{CDCl}_{3}$

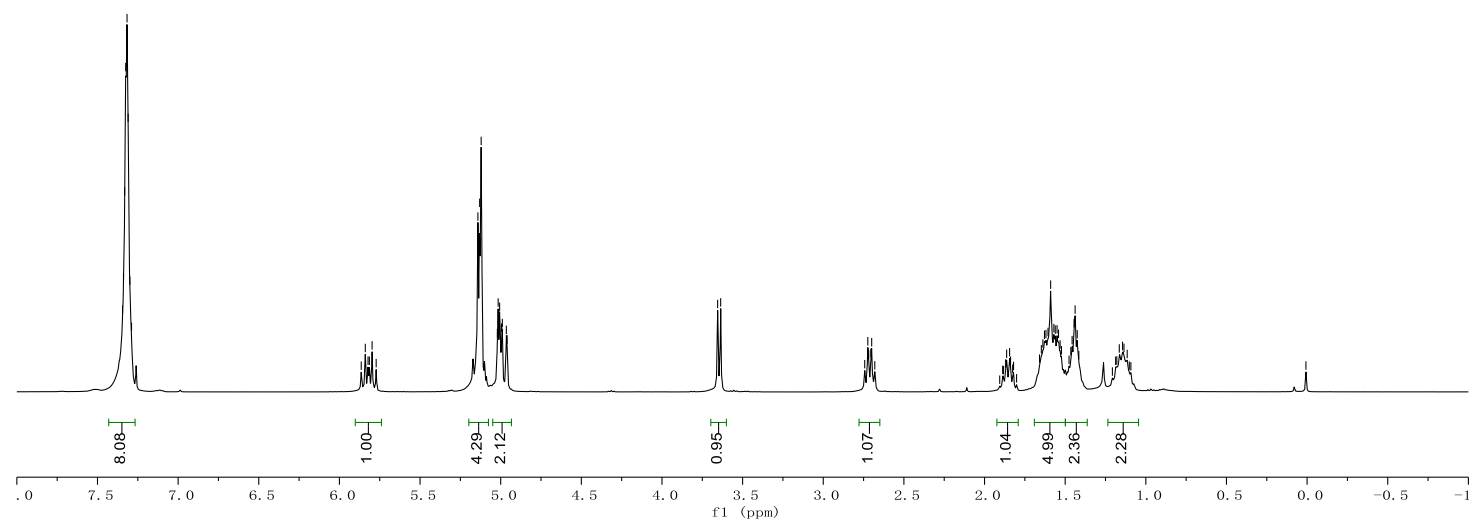

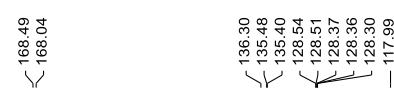

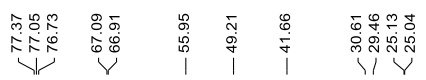<smiles>C=C[C@H](C1CCCC1)C(C(=O)OCc1ccccc1)C(=O)OCc1ccccc1</smiles>

$3 f$

${ }^{13} \mathrm{C}$-NMR $100 \mathrm{MHz}^{\mathrm{CDCl}}{ }_{3}$

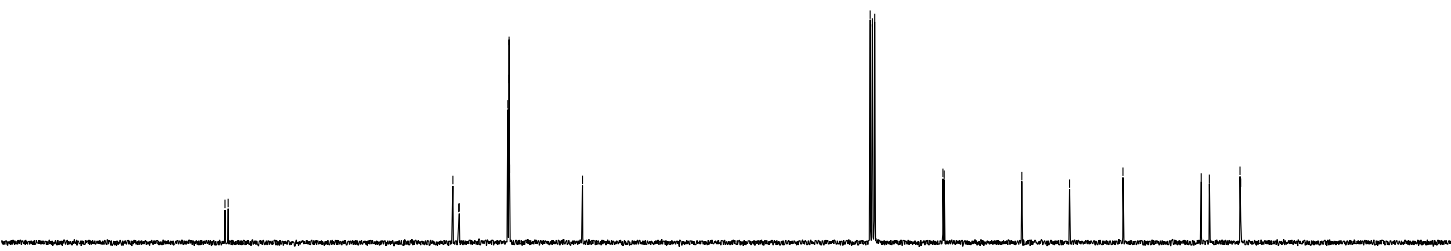

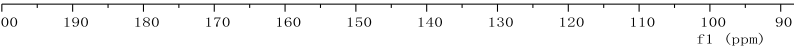




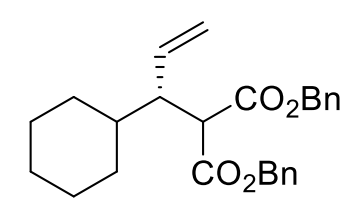

$3 \mathrm{~g}$

${ }^{1} \mathrm{H}-\mathrm{NMR} 400 \mathrm{MHz} \mathrm{CDCl}_{3}$

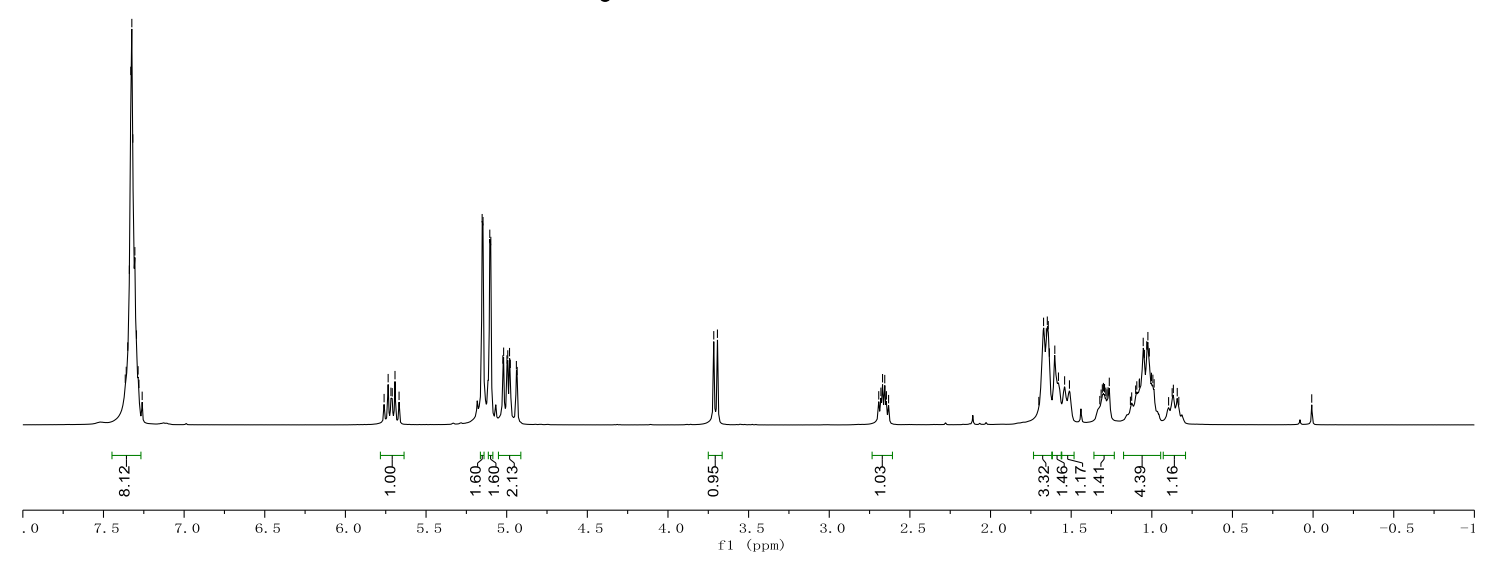

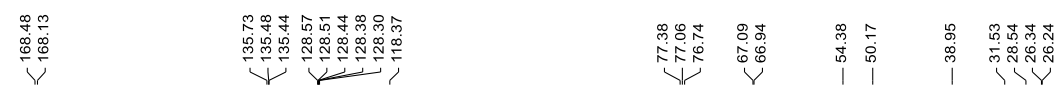<smiles>C=C[C@H](C1CCCCC1)C(C(=O)OCc1ccccc1)C(=O)OCc1ccccc1</smiles>

$3 \mathrm{~g}$

${ }^{13} \mathrm{C}-\mathrm{NMR}$ 100MHz $\mathrm{CDCl}_{3}$

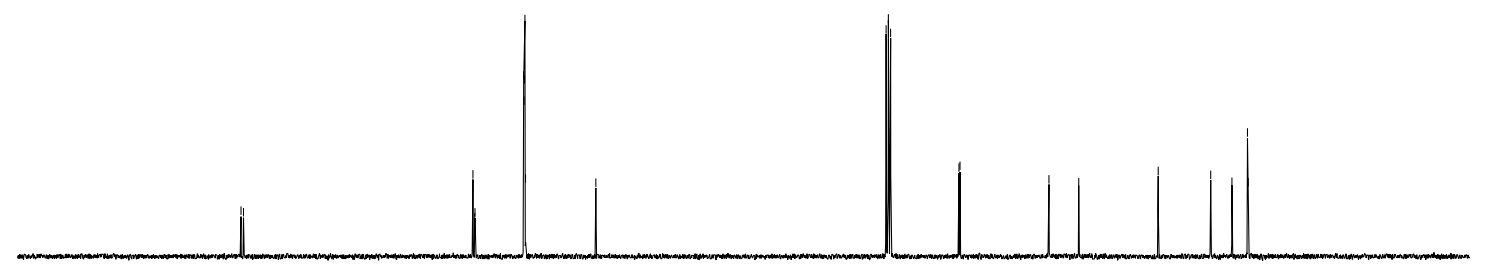

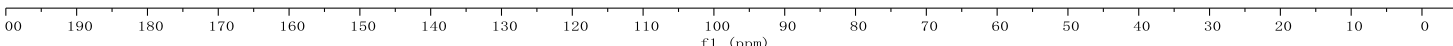


<smiles>C=C[C@H](COC(C)(C)C)C(C(C)=O)C(=O)OC</smiles>

$3 \mathrm{~h}$

${ }^{1} \mathrm{H}-\mathrm{NMR}$ 400MHz $\mathrm{CDCl}_{3}$

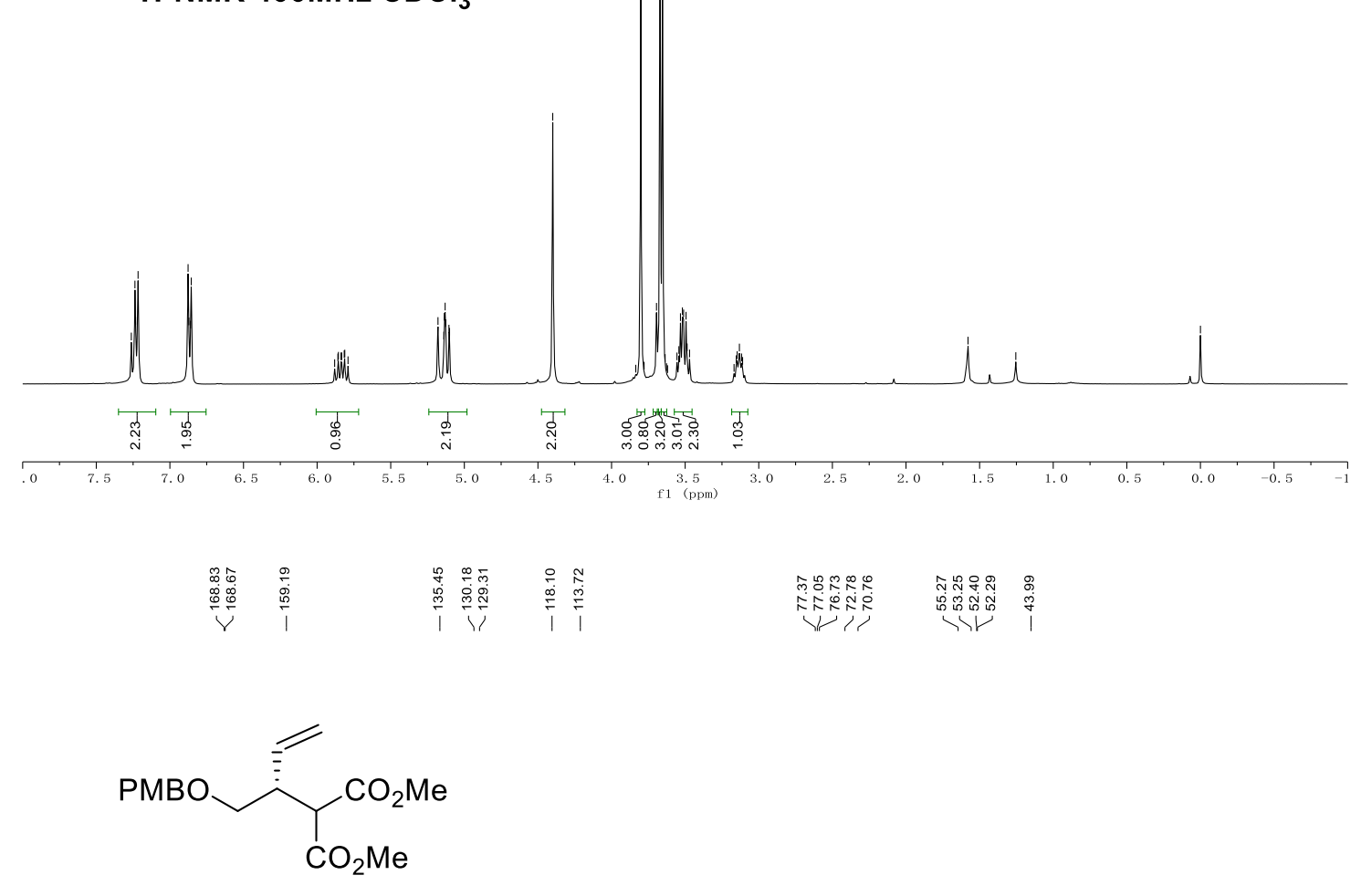

$3 \mathrm{~h}$

${ }^{13} \mathrm{C}-\mathrm{NMR} 100 \mathrm{MHz} \mathrm{CDCl}_{3}$

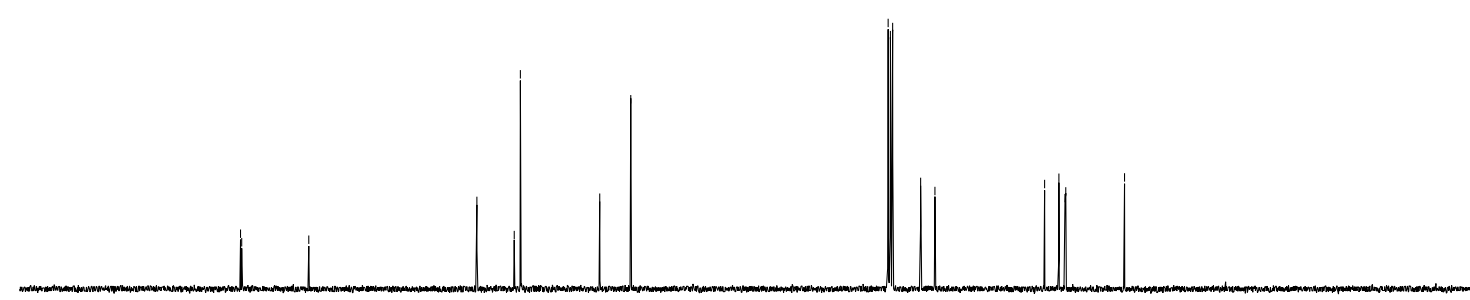


<smiles>C=CC(C(C)=O)[C@H](C=C)CCCCOC(C)(C)C</smiles>

$3 \mathbf{i}$

${ }^{1} \mathrm{H}-\mathrm{NMR}$ 400MHz $\mathrm{CDCl}_{3}$

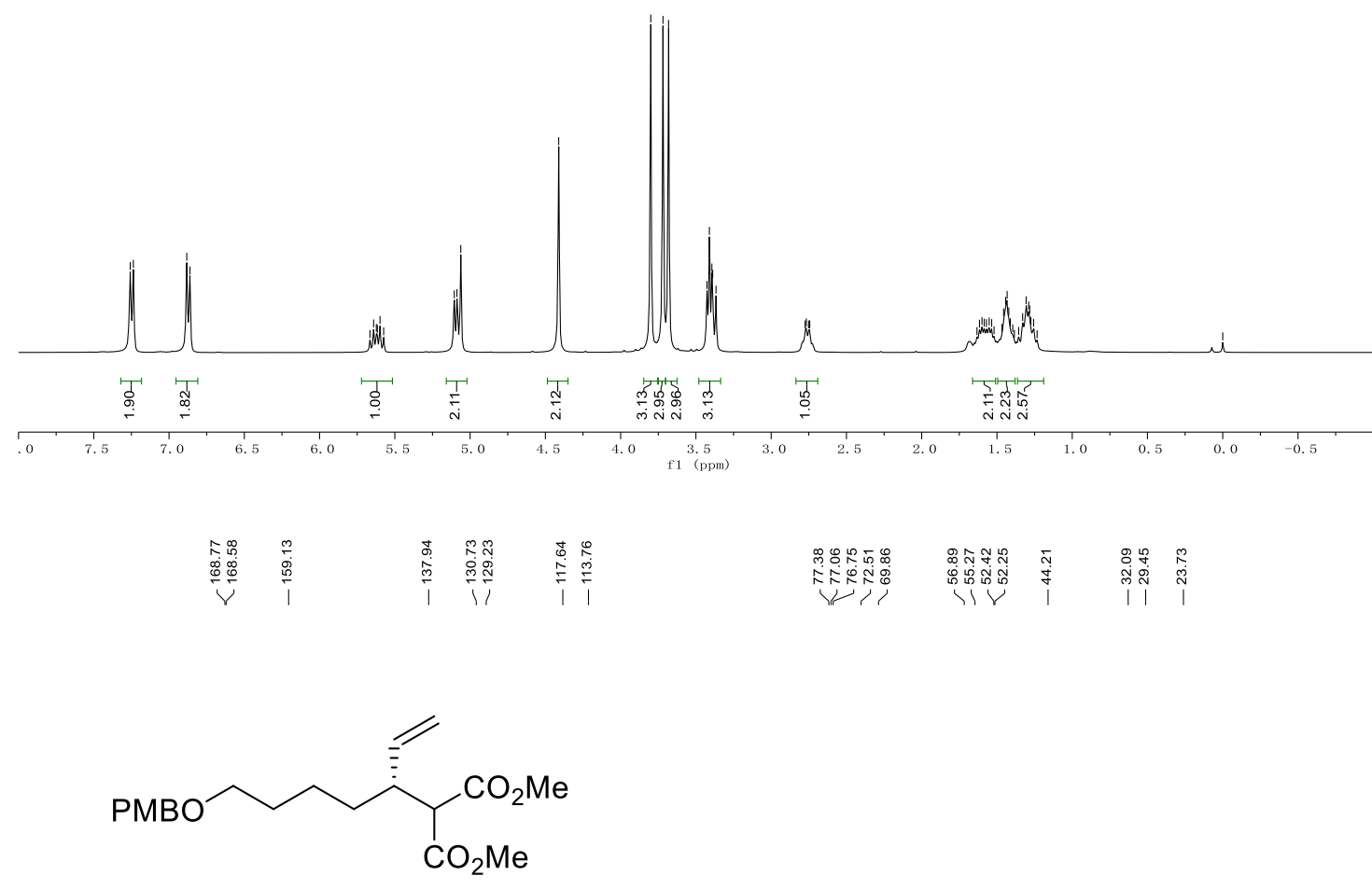

$3 \mathbf{i}$

${ }^{13} \mathrm{C}$-NMR $100 \mathrm{MHz} \mathrm{CDCl}_{3}$

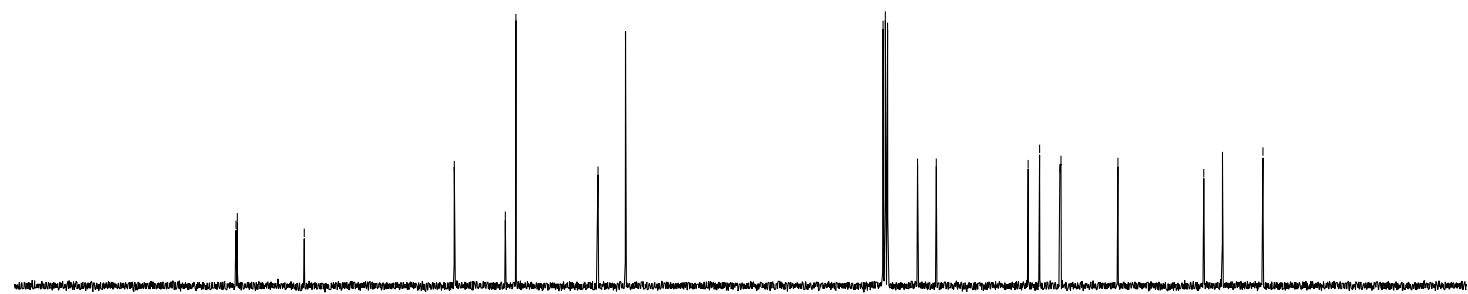


<smiles>C=C[C@H](CCCCCCOC(C)(C)C)C(C(C)=O)C(C)=O</smiles>

3j

${ }^{1} \mathrm{H}-\mathrm{NMR} 400 \mathrm{MHz} \mathrm{CDCl}_{3}$

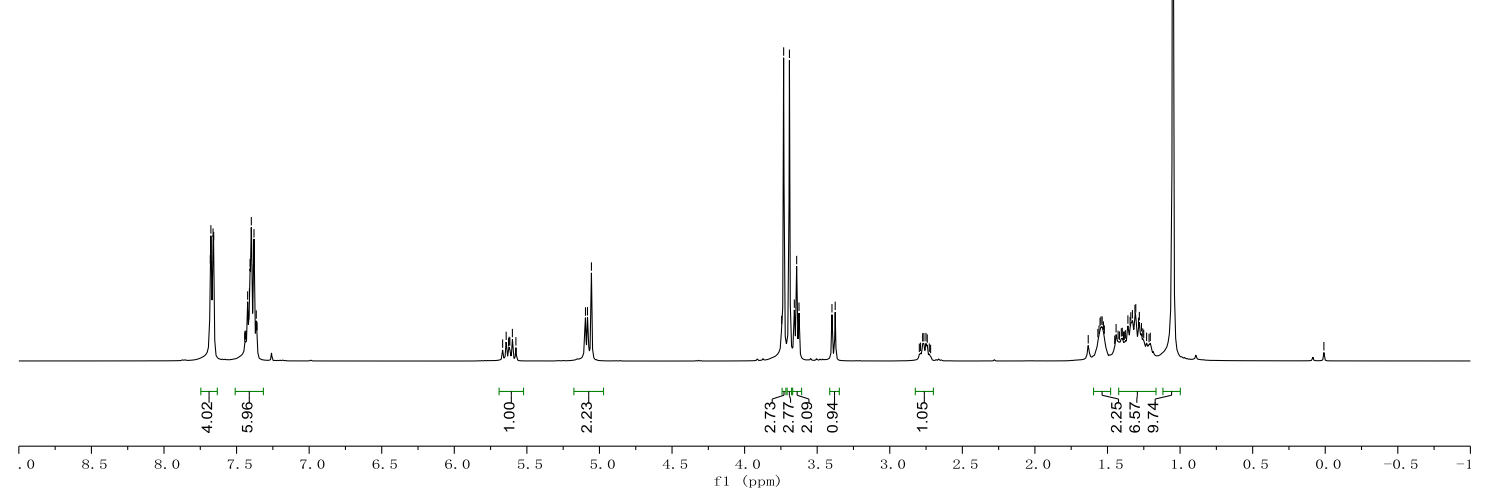<smiles>C=C[C@H](CCCCCCOC(C)(C)C)C(C(C)=O)C(C)=O</smiles>

3j

${ }^{13} \mathrm{C}-\mathrm{NMR} 100 \mathrm{MHz} \mathrm{CDCl}_{3}$

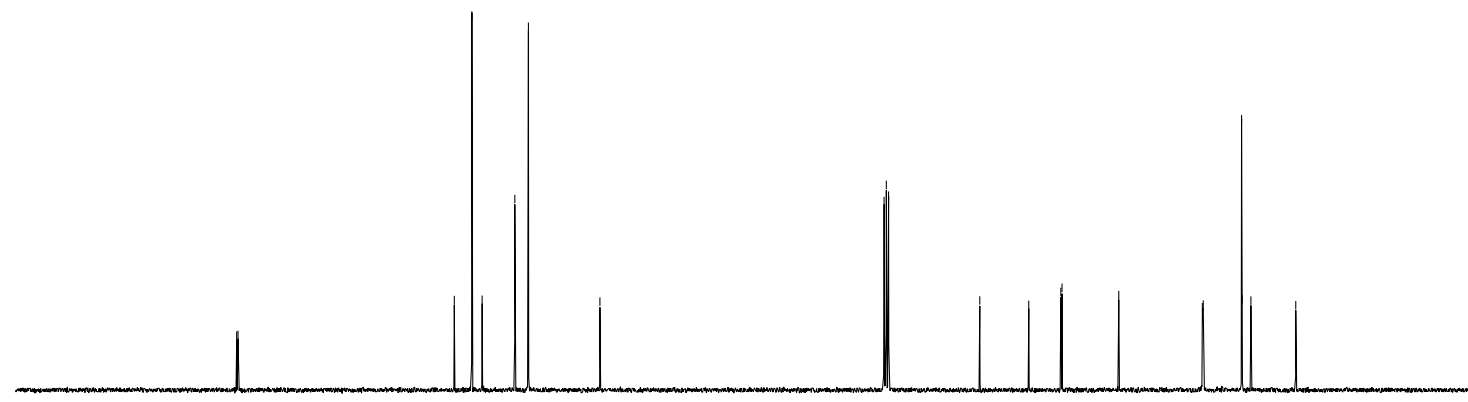

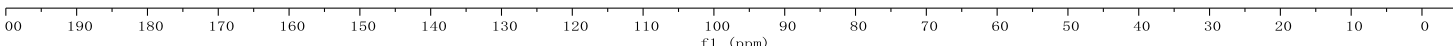


<smiles></smiles>

${ }^{1} \mathrm{H}-\mathrm{NMR} 400 \mathrm{MHz} \mathrm{CDCl}_{3}$

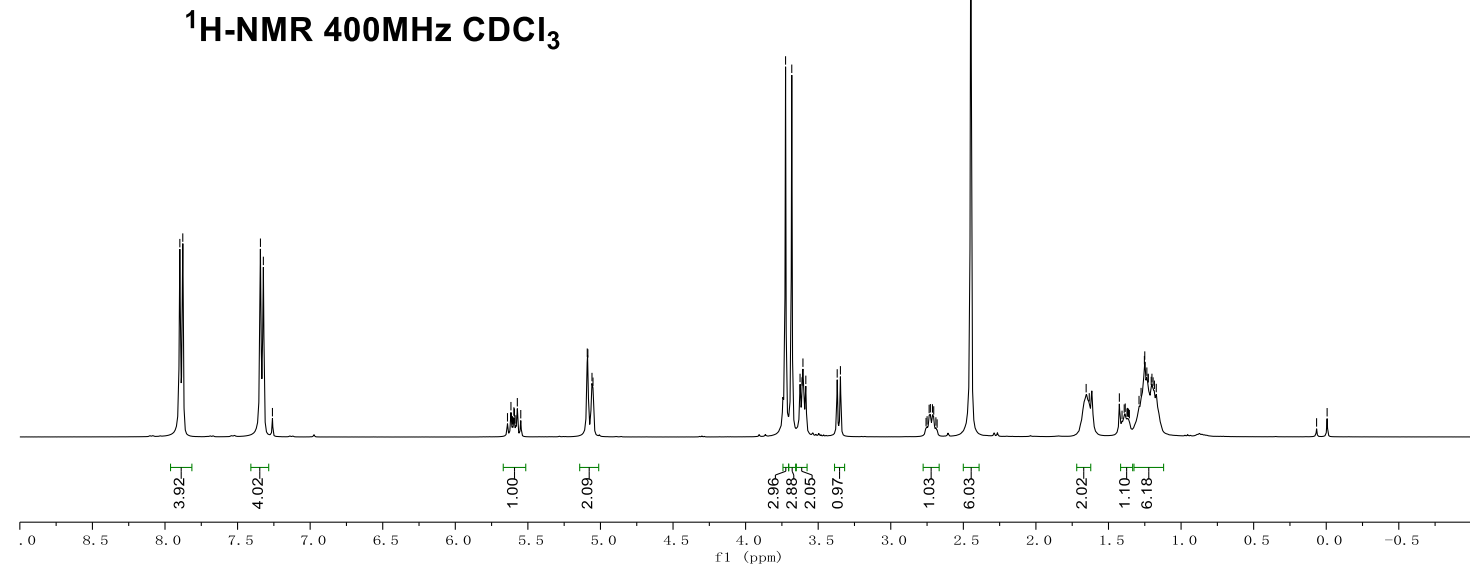<smiles>C=C[C@H](CCCCCCNC(C)(C)C)C(C(C)=O)C(C)=O</smiles>

3k

${ }^{13} \mathrm{C}-\mathrm{NMR}$ 100MHz $\mathrm{CDCl}_{3}$

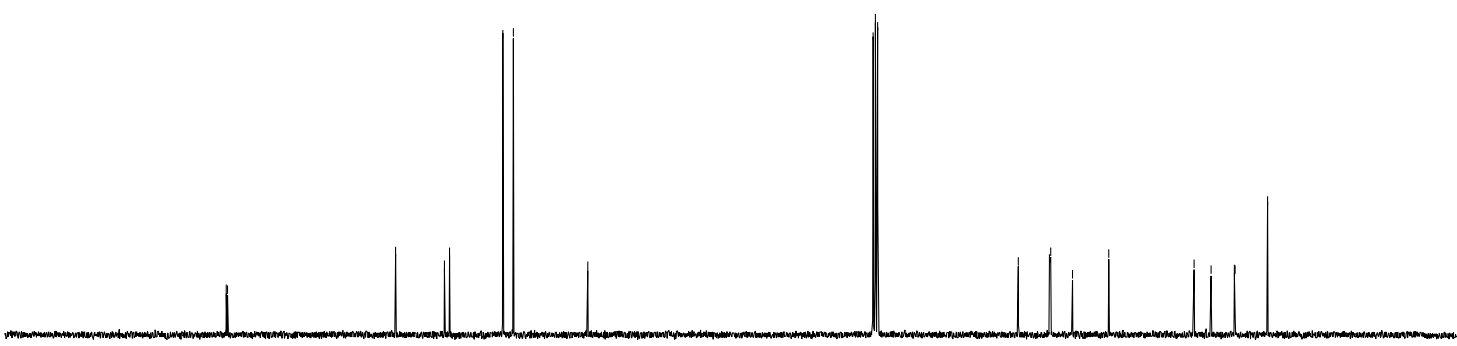

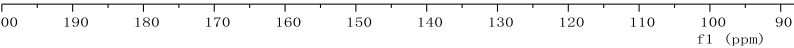


<smiles>C=CCCC(C=C)C(C(=O)OCc1ccccc1)C(=O)OCc1ccccc1</smiles>

31

${ }^{1} \mathrm{H}-\mathrm{NMR} 400 \mathrm{MHz} \mathrm{CDCl}_{3}$

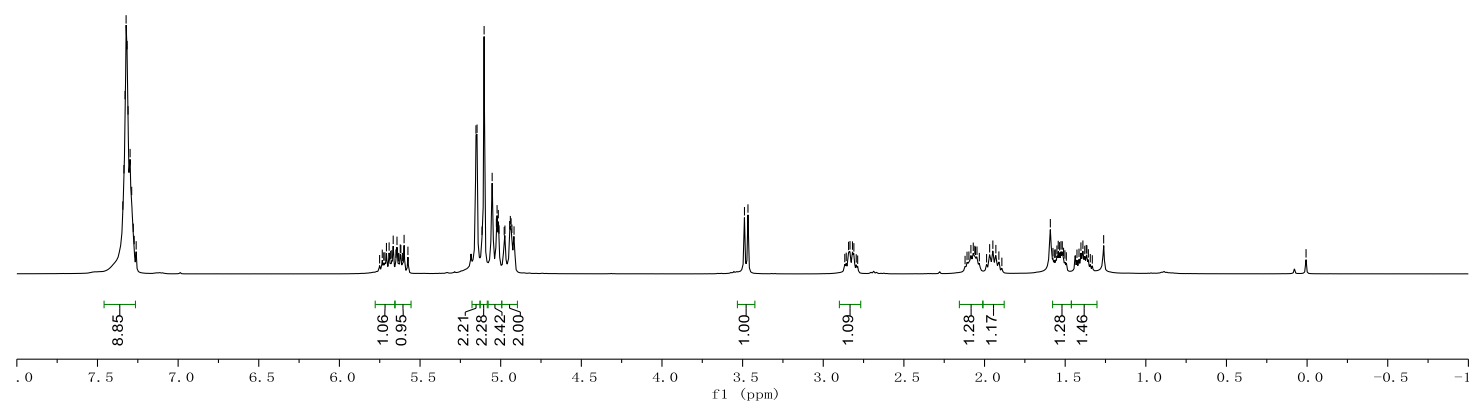

V)<smiles>C=CCC[C@H](C=C)C(C(=O)OBr)C(=O)Oc1ccccc1</smiles>

3I

${ }^{13} \mathrm{C}-\mathrm{NMR} 100 \mathrm{MHz} \mathrm{CDCl}_{3}$

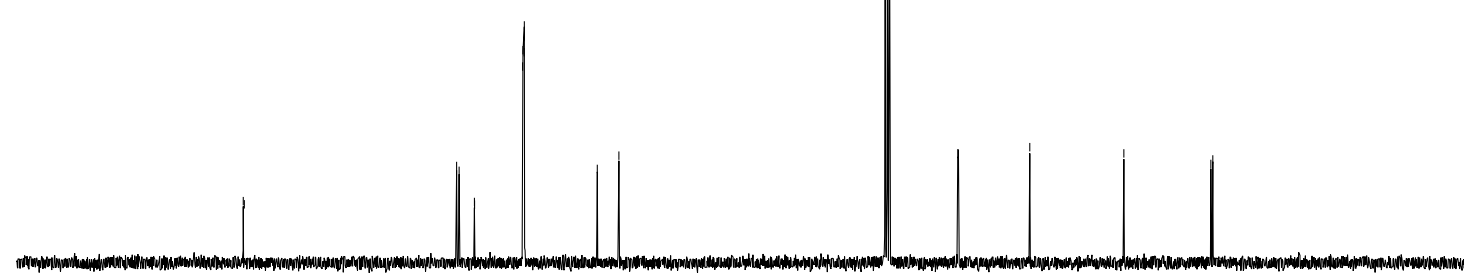

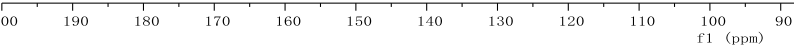


<smiles>C=C[C@H](C[C@@H](C)CCC=C(C)C)C(C(=O)OCc1ccccc1)C(=O)Oc1ccccc1</smiles>

${ }^{1} \mathrm{H}-\mathrm{NMR}$ 400MHz $\mathrm{CDCl}_{3}$

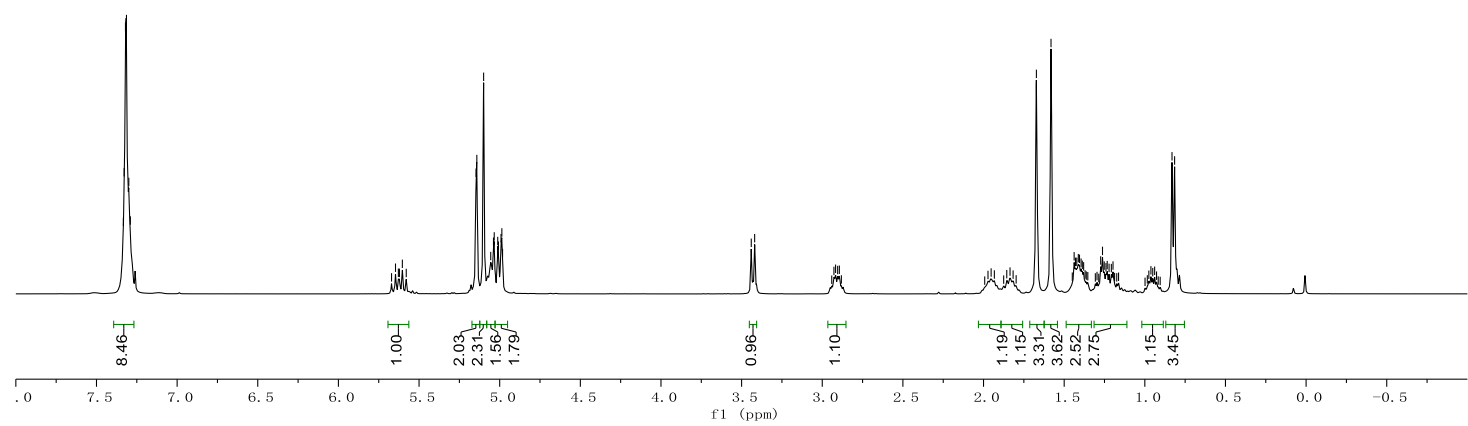

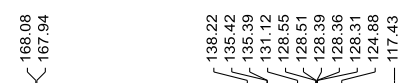

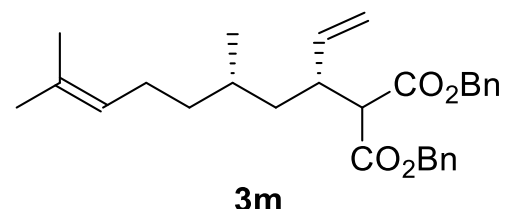

${ }^{13} \mathrm{C}-\mathrm{NMR} 100 \mathrm{MHz} \mathrm{CDCl}_{3}$

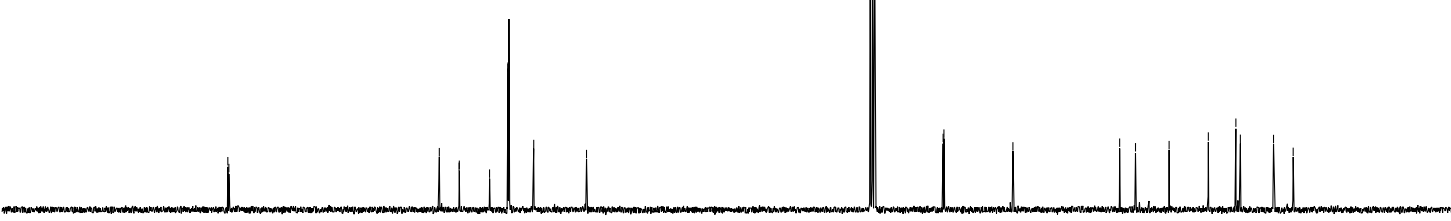

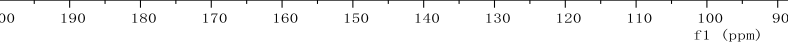


<smiles>C=CC(C[C@@H](C)CCC=C(C)C)C(C(=O)OCc1ccccc1)C(=O)OCc1ccccc1</smiles>

${ }^{1} \mathrm{H}-\mathrm{NMR}$ 400MHz $\mathrm{CDCl}_{3}$

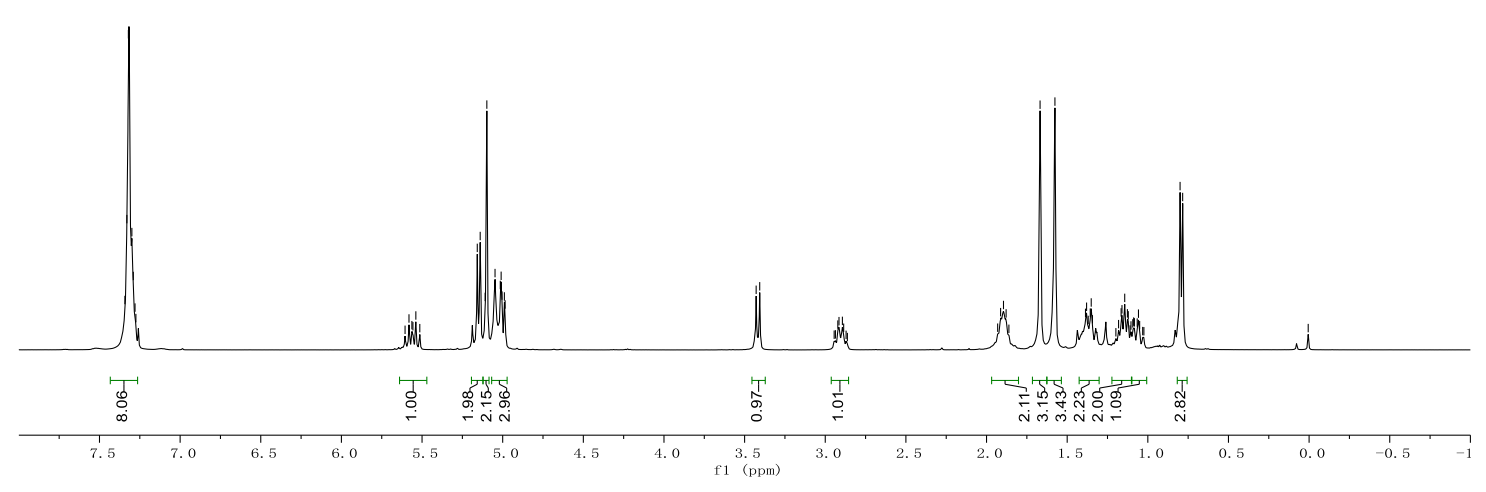

$\underbrace{\frac{1}{2}}$<smiles>C=CC(C[C@@H](C)CCC=C(C)C)C(C(=O)OCc1ccccc1)C(=O)OCc1ccccc1</smiles>

${ }^{13} \mathrm{C}$-NMR $100 \mathrm{MHz}^{\mathrm{CDCl}_{3}}$

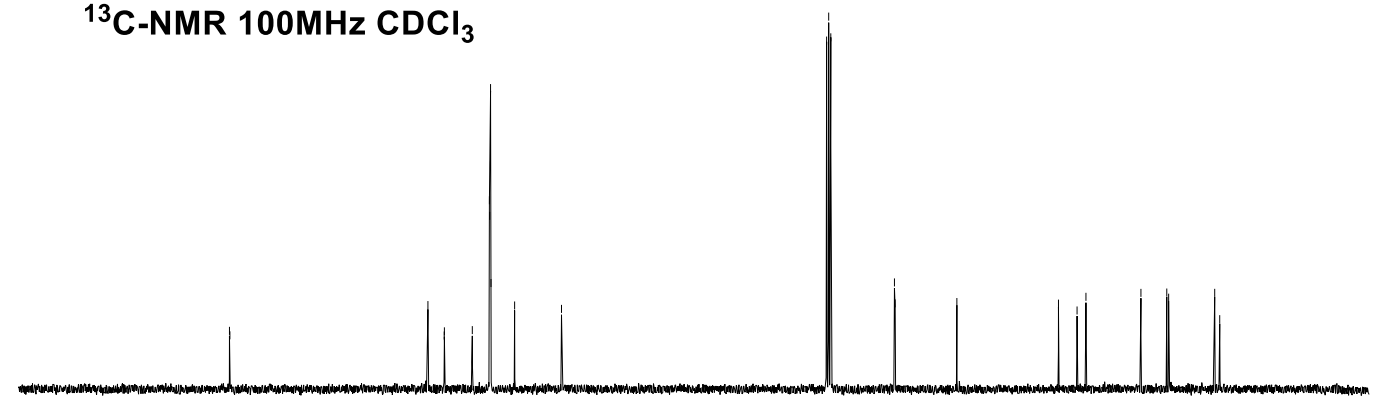




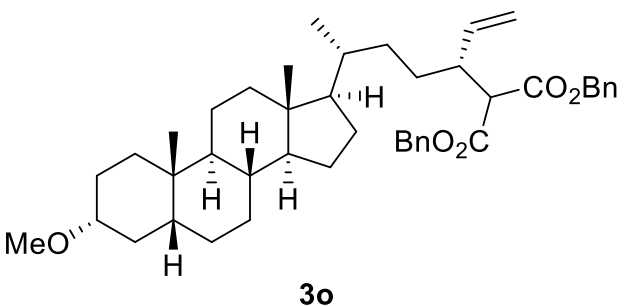

${ }^{1} \mathrm{H}-\mathrm{NMR}$ 400MHz $\mathrm{CDCl}_{3}$
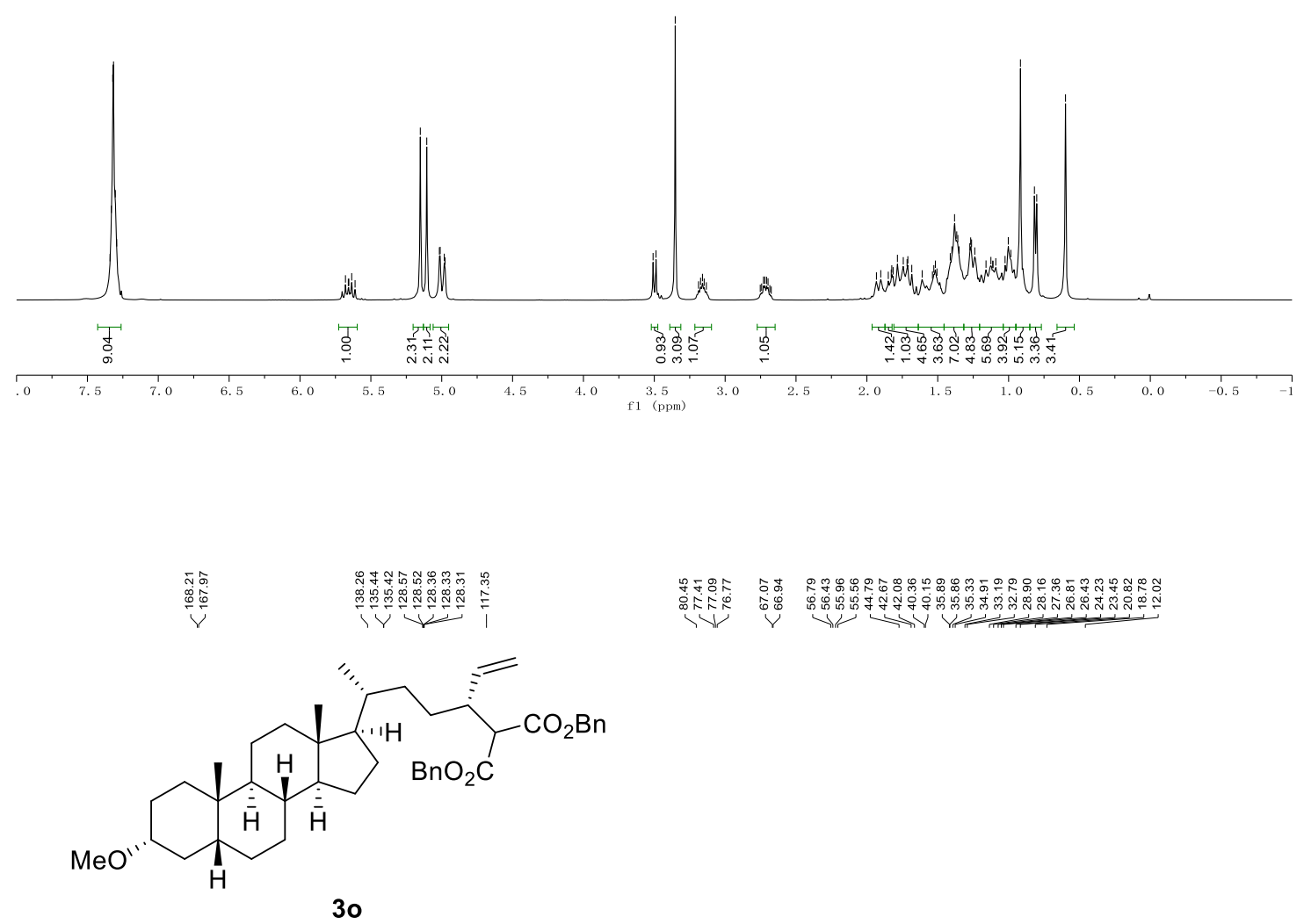

${ }^{13} \mathrm{C}$-NMR $100 \mathrm{MHz} \mathrm{CDCl}_{3}$

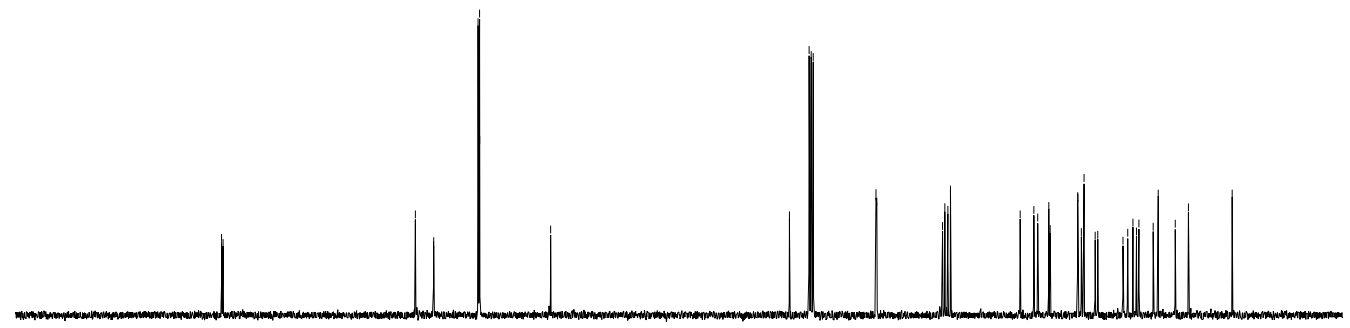

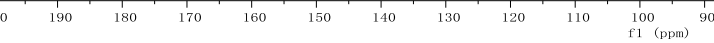


<smiles>C=C[C@H](CCc1ccccc1)[C@H](C(=O)CCC)C(=O)OCC</smiles>

$3 p$

${ }^{1} \mathrm{H}-\mathrm{NMR}$ 400MHz CDCl3

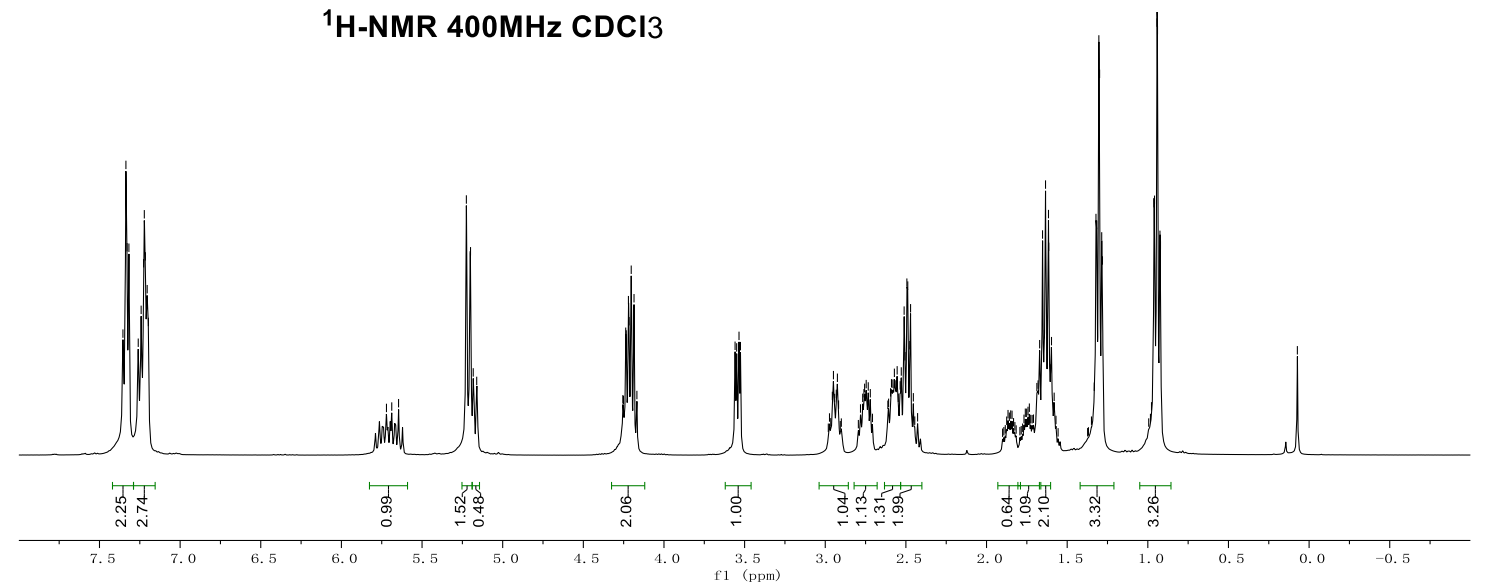<smiles>C=C[C@H](CCc1ccccc1)C(C(=O)CCC)C(=O)OCC</smiles>

$3 p$

${ }^{13} \mathrm{C}-\mathrm{NMR} 100 \mathrm{MHz} \mathrm{CDCl}_{3}$

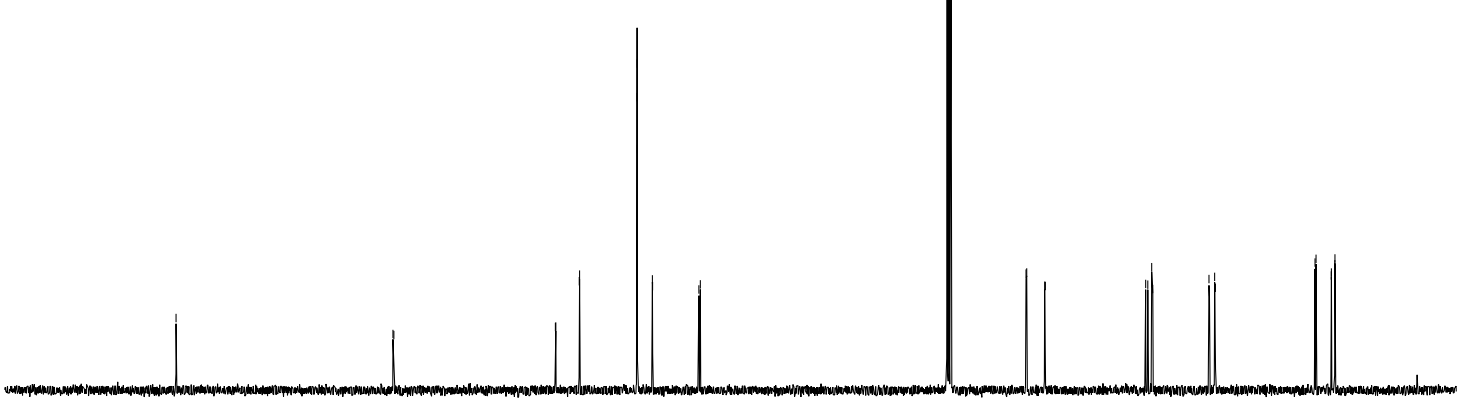

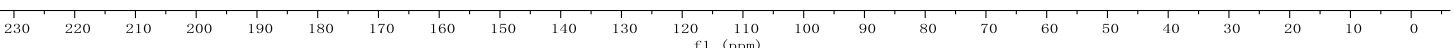




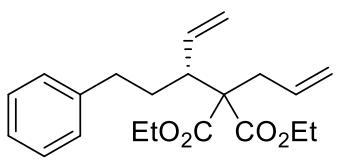

$3 q$

${ }^{1} \mathrm{H}-\mathrm{NMR} 400 \mathrm{MHz} \mathrm{CDCl}_{3}$
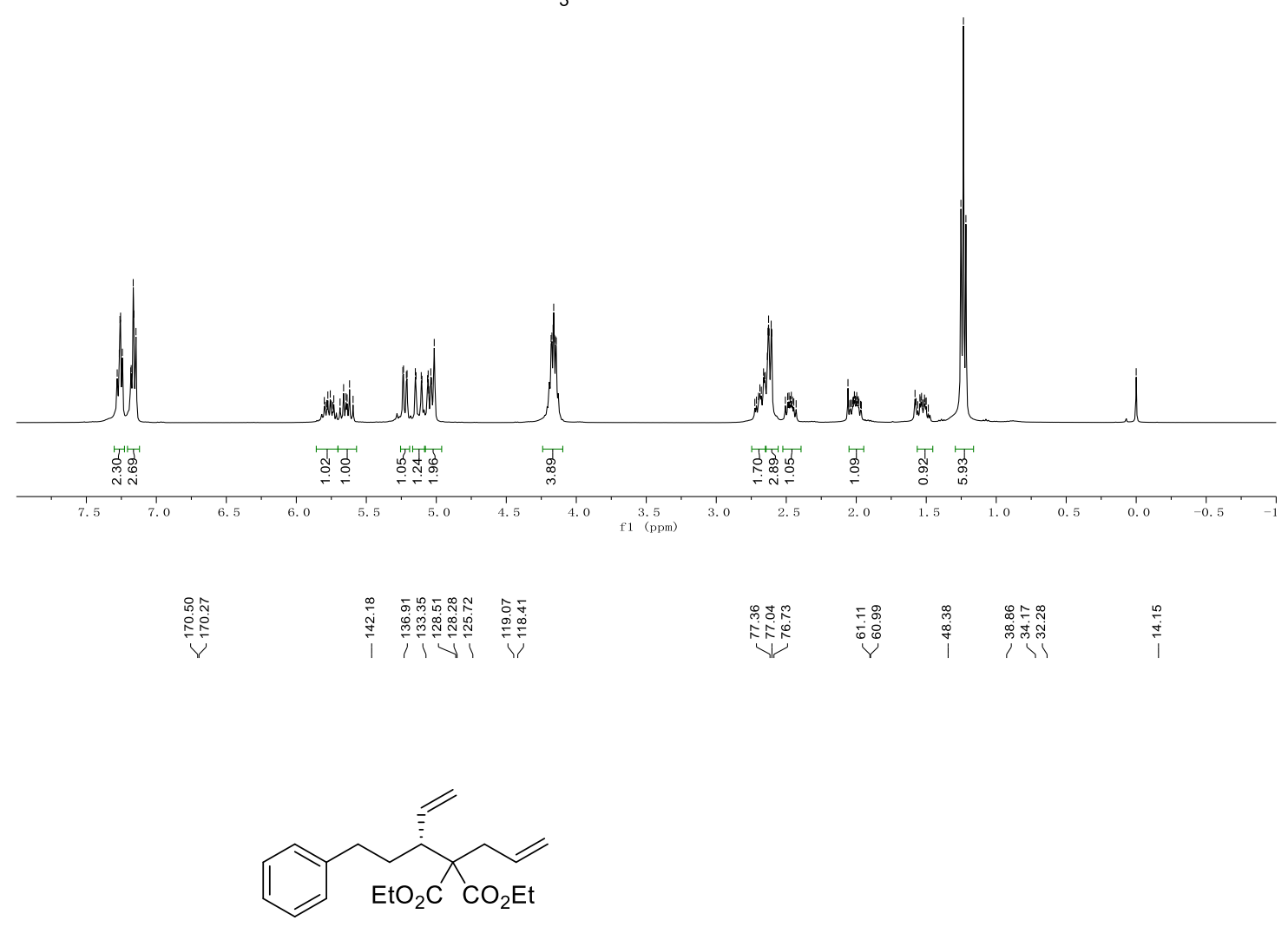

$3 q$

${ }^{13} \mathrm{C}-\mathrm{NMR} 100{\mathrm{MHz} \mathrm{CDCl}_{3}}$

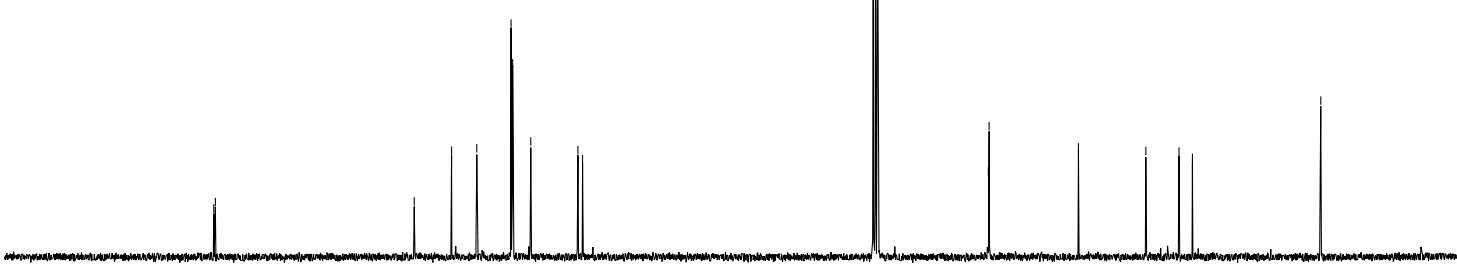

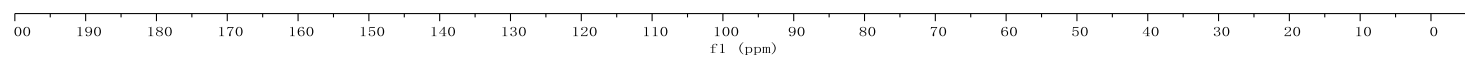




\section{HPLC Data}<smiles>C=C[C@H](CCc1ccccc1)C(C(C)=O)C(C)=O</smiles>

$3 a$

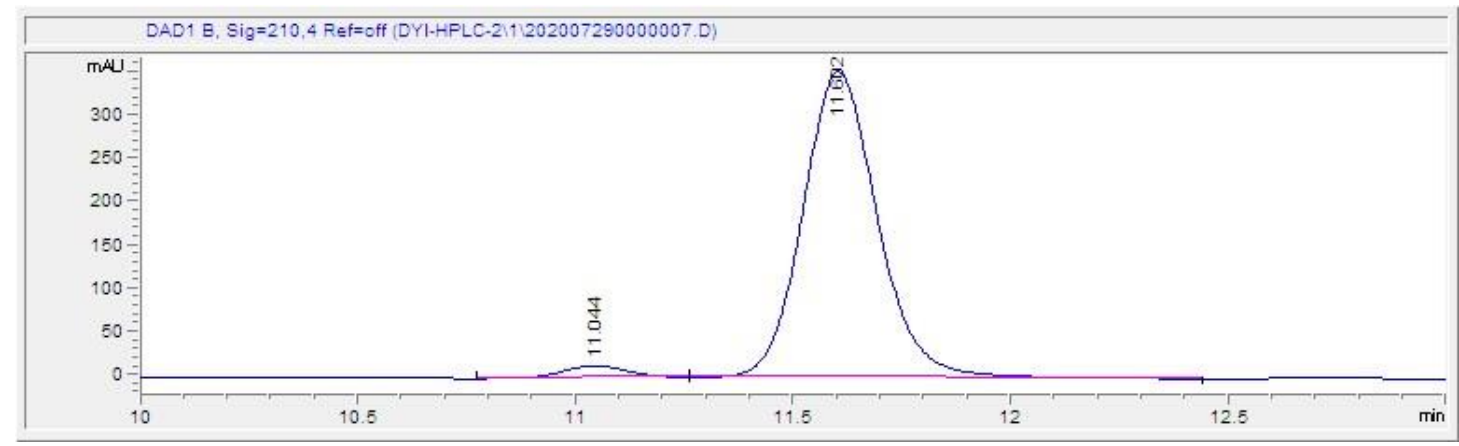

\begin{tabular}{|c|c|c|c|c|c|c|}
\hline$\#$ & Time & Area & Height & Width & Area\% & Symmetry \\
\hline 1 & 11.044 & 143.4 & 13.8 & 0.1636 & 3.280 & 0.976 \\
\hline 2 & 11.602 & 4229 & 353.4 & 0.1836 & 96.720 & 0.848 \\
\hline
\end{tabular}

Racemic:

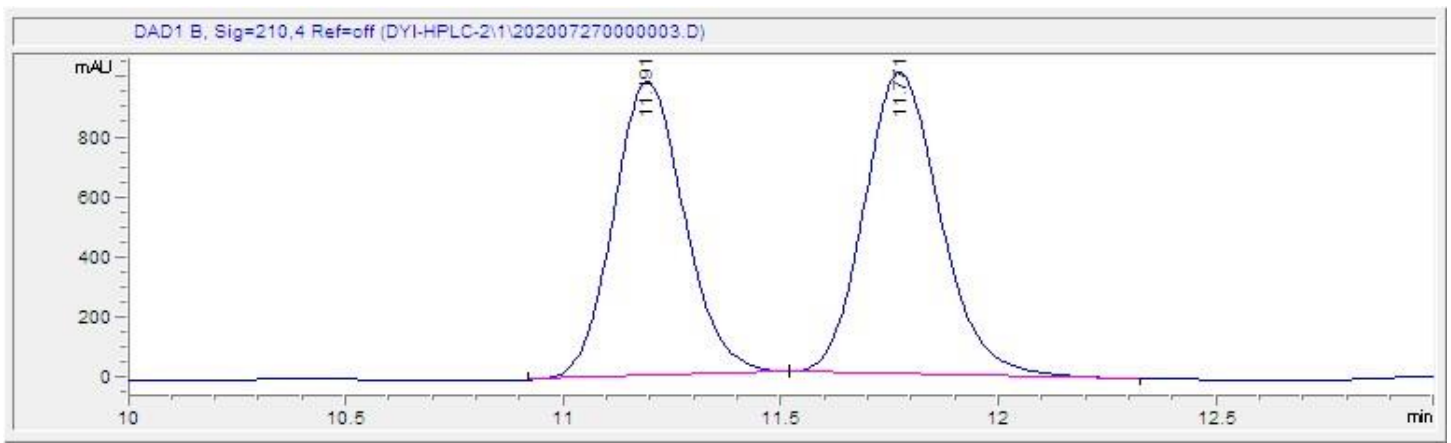

\begin{tabular}{|c|c|c|c|c|c|c|}
\hline \# & Time & Area & Height & Width & Area\% & Symmetry \\
\hline 1 & 11.191 & 11096.3 & 982.1 & 0.1758 & 47.619 & 0.877 \\
\hline 2 & 11.771 & 12205.9 & 1002.8 & 0.188 & 52.381 & 0.826 \\
\hline
\end{tabular}


<smiles>C=C[C@@H](Cc1ccccc1)C(C(C)=O)C(C)=O</smiles>

3b

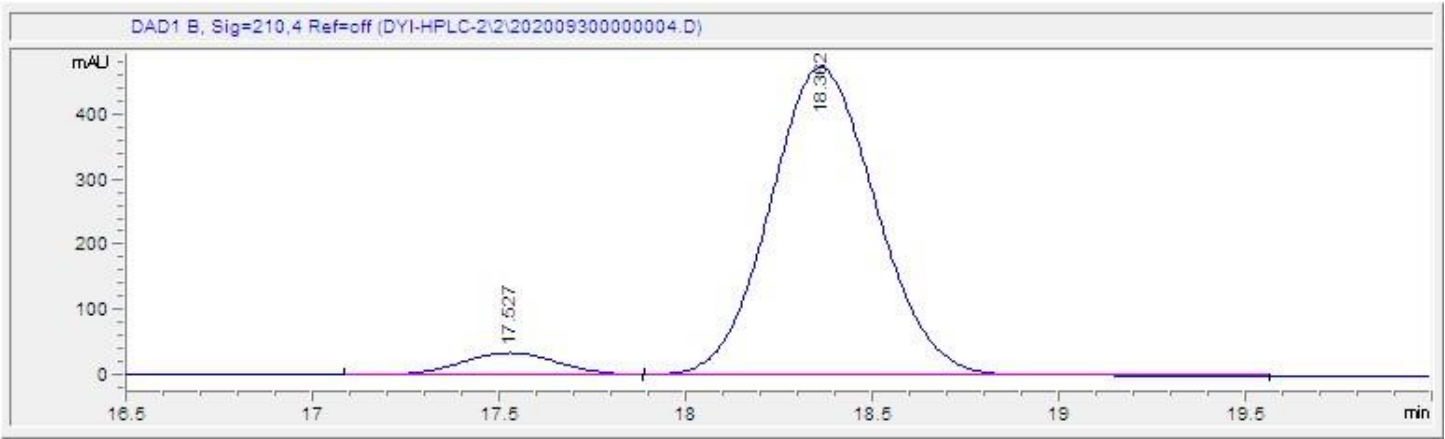

\begin{tabular}{|c|c|c|c|c|c|c|}
\hline$\#$ & Time & Area & Height & Width & Area\% & Symmetry \\
\hline 1 & 17.527 & 607.5 & 33.9 & 0.2808 & 6.084 & 0.988 \\
\hline 2 & 18.362 & 9378.1 & 474.1 & 0.3088 & 93.916 & 0.891 \\
\hline
\end{tabular}

Racemic:

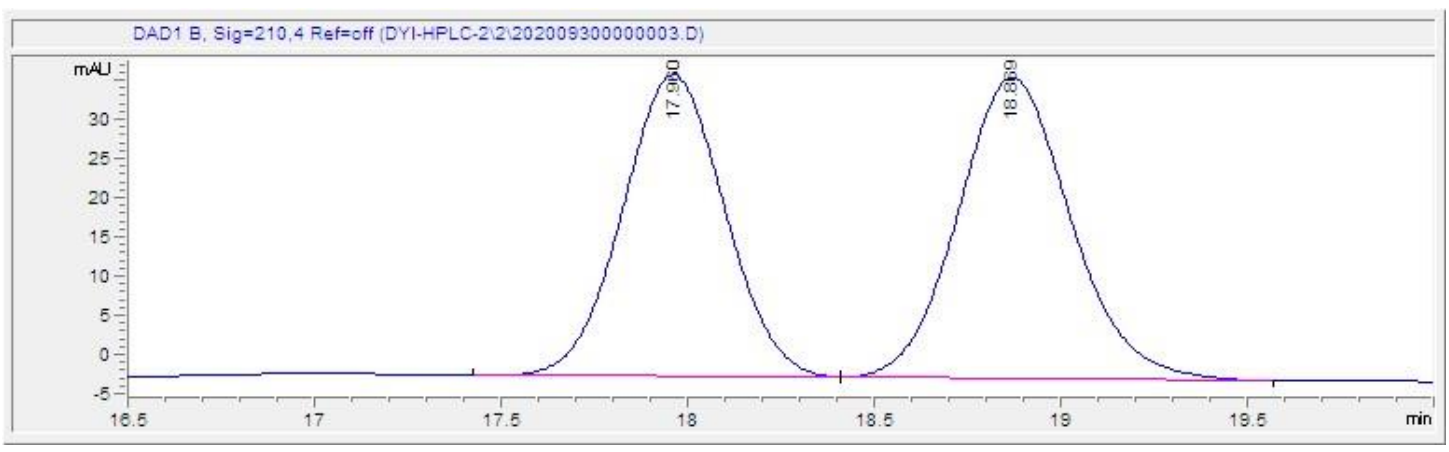

\begin{tabular}{|c|c|c|c|c|c|c|} 
\# & \multicolumn{1}{c}{ Time } & Area & Height & Width & \multicolumn{1}{c}{ Area\% } & \multicolumn{1}{c}{ Symmetry } \\
\hline 1 & 17.96 & 732.4 & 38.5 & 0.2961 & 47.555 & 0.961 \\
\hline 2 & 18.869 & 807.7 & 38.4 & 0.3253 & 52.445 & 0.883 \\
\hline
\end{tabular}


$\overbrace{\mathrm{CO}_{2} \mathrm{Bn}}^{\mathrm{CO}_{2} \mathrm{Bn}}$

$3 c$

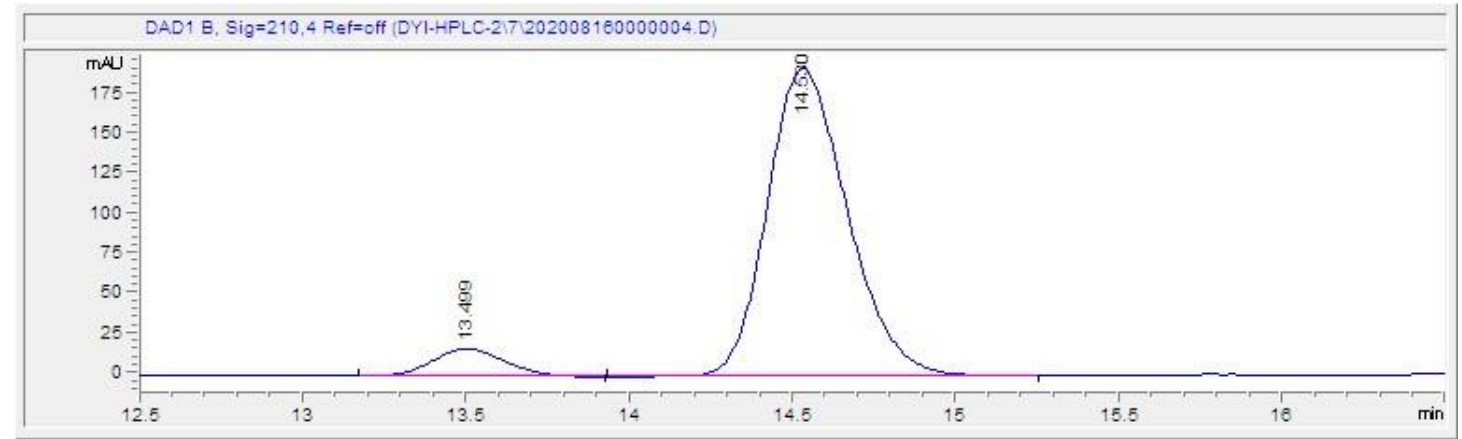

\begin{tabular}{|c|c|c|c|c|c|c|}
\hline$\#$ & Time & Area & Height & Width & Area\% & Symmetry \\
\hline 1 & 13.499 & 260.6 & 17 & 0.239 & 7.363 & 0.846 \\
\hline 2 & 14.53 & 3278.8 & 192 & 0.2632 & 92.637 & 0.762 \\
\hline
\end{tabular}

\section{Racemic:}

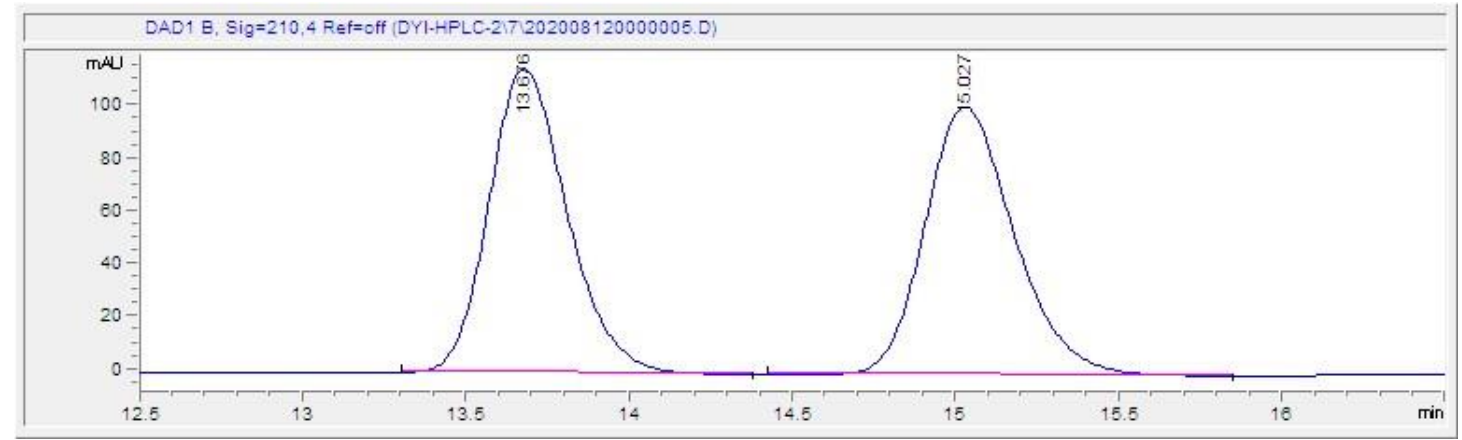

\begin{tabular}{|c|c|c|c|c|c|c|} 
\# & Time & Area & Height & Width & \multicolumn{1}{c}{ Area\% } & Symmetry \\
\hline 1 & 13.676 & 1923.6 & 115.3 & 0.2566 & 49.773 & 0.777 \\
\hline 2 & 15.027 & 1941.2 & 101.8 & 0.2926 & 50.227 & 0.77 \\
\hline
\end{tabular}


<smiles>C=C[C@H](CCC)C(C(=O)OCc1ccccc1)C(=O)OCc1ccccc1</smiles>

$3 d$

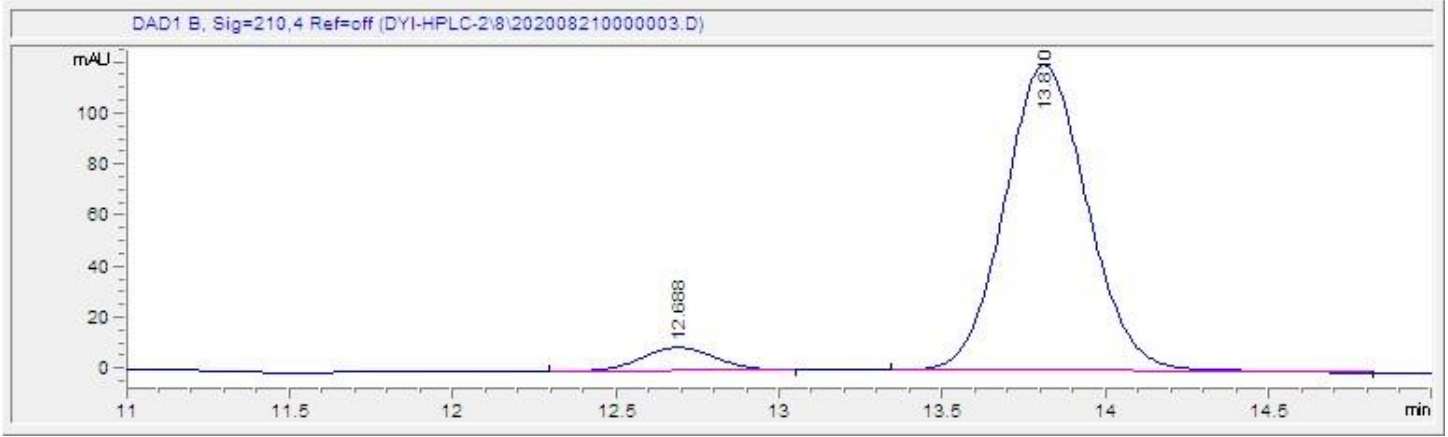

\begin{tabular}{|c|c|c|c|c|c|c|} 
\# & \multicolumn{1}{c}{ Time } & Area & Height & Width & \multicolumn{1}{c}{ Area\% } & Symmetry \\
\hline 1 & 12.688 & 142.1 & 9.1 & 0.2446 & 6.261 & 0.989 \\
\hline 2 & 13.81 & 2127.8 & 119.9 & 0.2771 & 93.739 & 0.882 \\
\hline
\end{tabular}

\section{Racemic:}

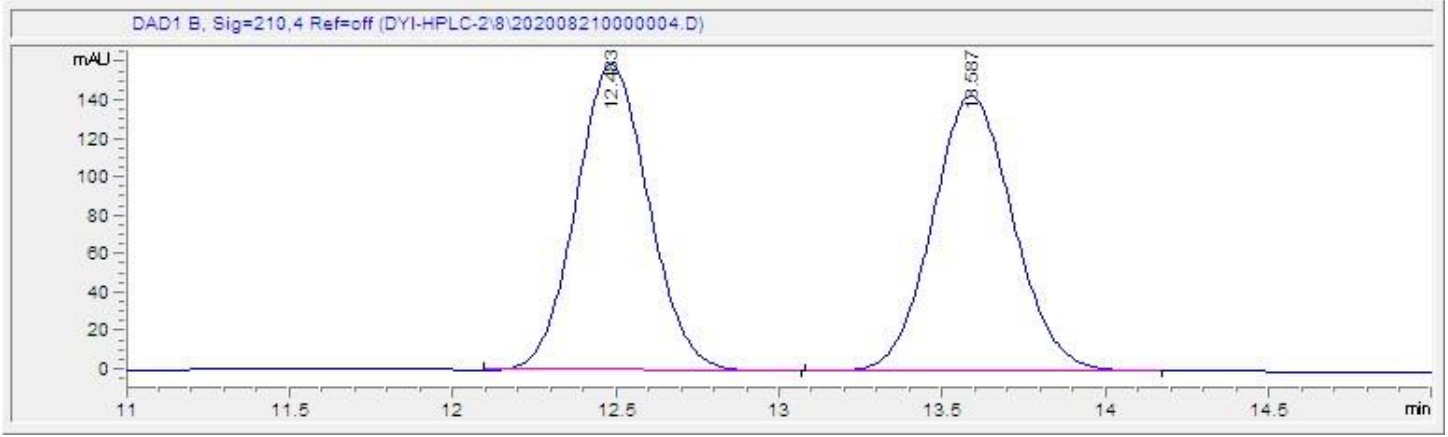

\begin{tabular}{|c|c|c|c|c|c|c|} 
\# & \multicolumn{1}{c}{ Time } & Area & Height & Width & \multicolumn{1}{c|}{ Area\% } & Symmetry \\
\hline 1 & 12.483 & 2468.1 & 159.4 & 0.2407 & 49.987 & 0.911 \\
\hline 2 & 13.587 & 2469.4 & 143.2 & 0.2672 & 50.013 & 0.906 \\
\hline
\end{tabular}


<smiles>C=C[C@@H](C1CC1)C(C(=O)OCc1ccccc1)C(=O)OCc1ccccc1</smiles>

$3 e$

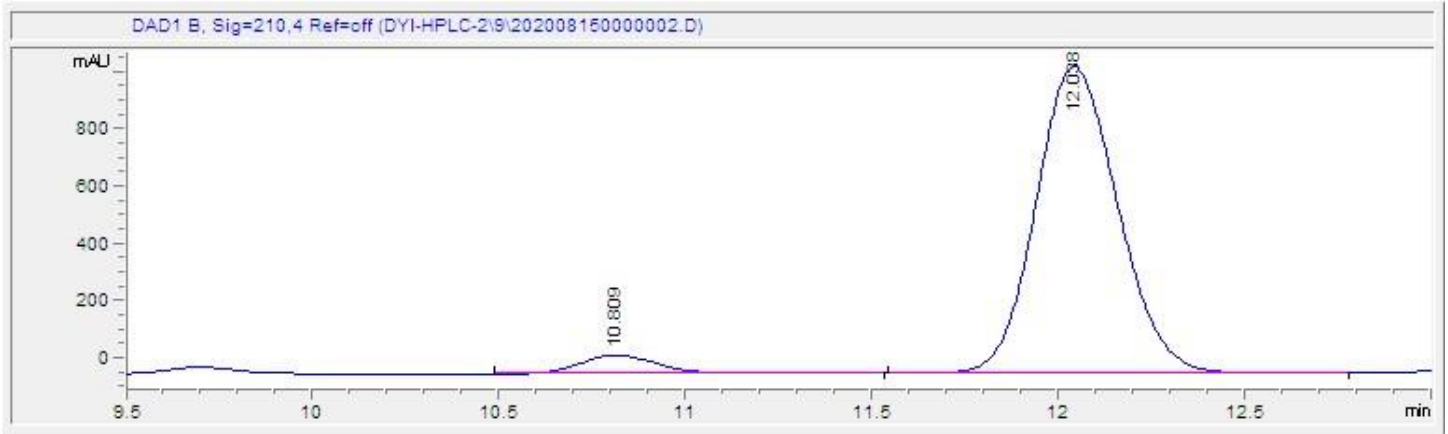

\begin{tabular}{|c|c|c|c|c|c|c|}
\multicolumn{1}{l}{ \# } & Time & Area & Height & Width & \multicolumn{1}{c|}{ Area\% } & \multicolumn{1}{c|}{ Symmetry } \\
\hline 1 & 10.809 & 942.5 & 65 & 0.2093 & 5.472 & 0.798 \\
\hline 2 & 12.038 & 16283.5 & 1069.6 & 0.2376 & 94.528 & 0.84 \\
\hline
\end{tabular}

\section{Racemic:}

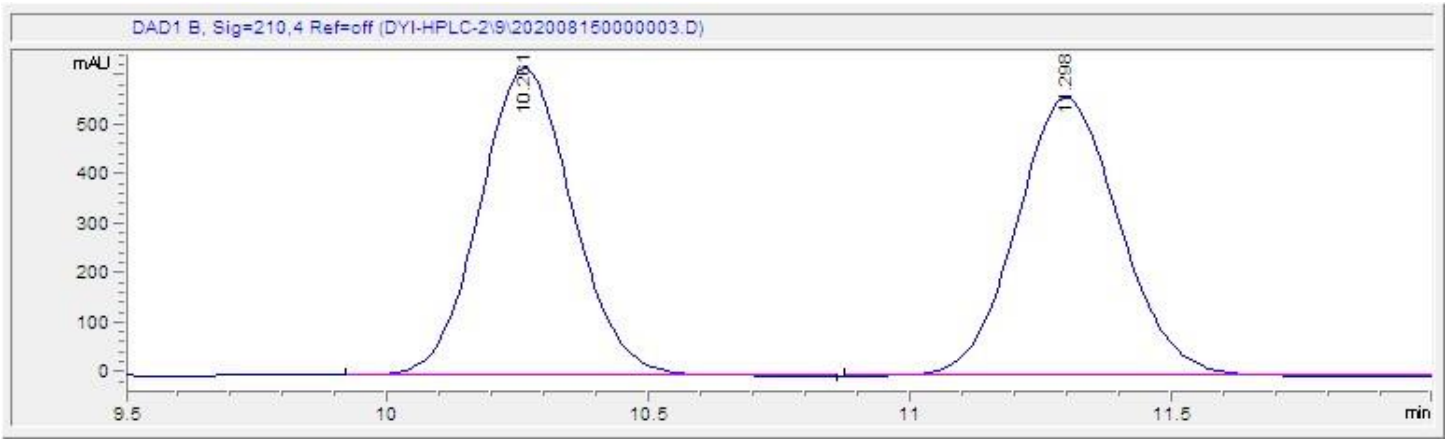

\begin{tabular}{|c|c|c|c|c|c|c|} 
\# & \multicolumn{1}{c}{ Time } & Area & Height & Width & \multicolumn{1}{c|}{ Area\% } & Symmetry \\
\hline 1 & 10.261 & 7670 & 620.5 & 0.1922 & 49.901 & 0.906 \\
\hline 2 & 11.298 & 7700.3 & 561.7 & 0.2119 & 50.099 & 0.894 \\
\hline
\end{tabular}




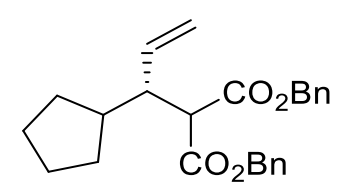

$3 f$

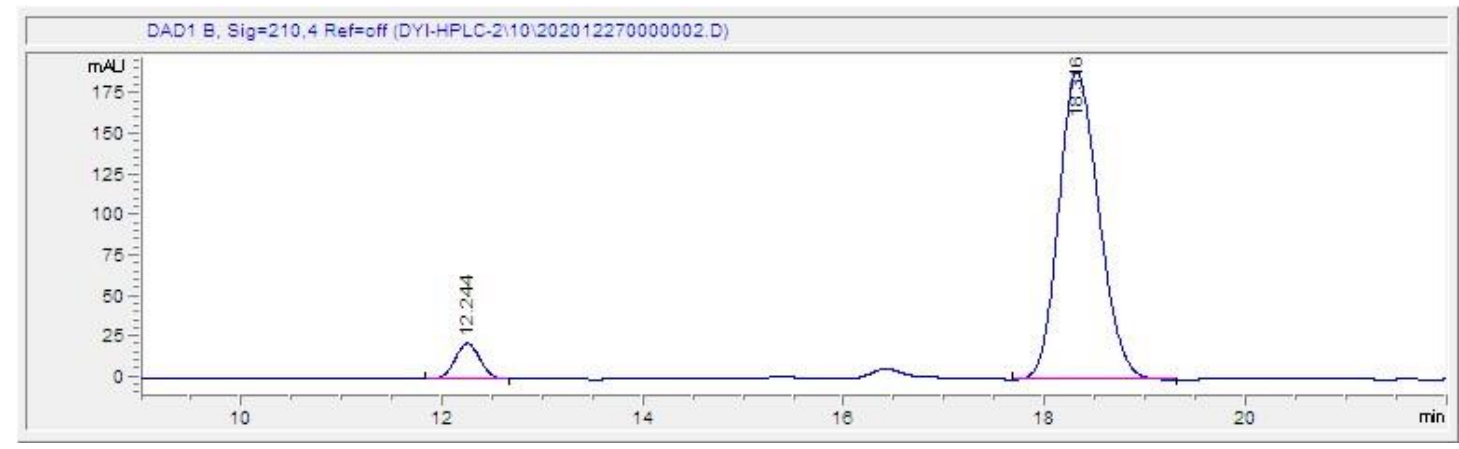

\begin{tabular}{|c|c|c|c|c|c|c|}
\multicolumn{1}{l}{ \# } & Time & \multicolumn{1}{c}{ Area } & Height & Width & \multicolumn{1}{c}{ Area\% } & \multicolumn{1}{c}{ Symmetry } \\
\hline 1 & 12.244 & 380.6 & 21.9 & 0.2703 & 6.722 & 0.952 \\
\hline 2 & 18.316 & 5281.7 & 190.1 & 0.4321 & 93.278 & 0.784 \\
\hline
\end{tabular}

\section{Racemic:}

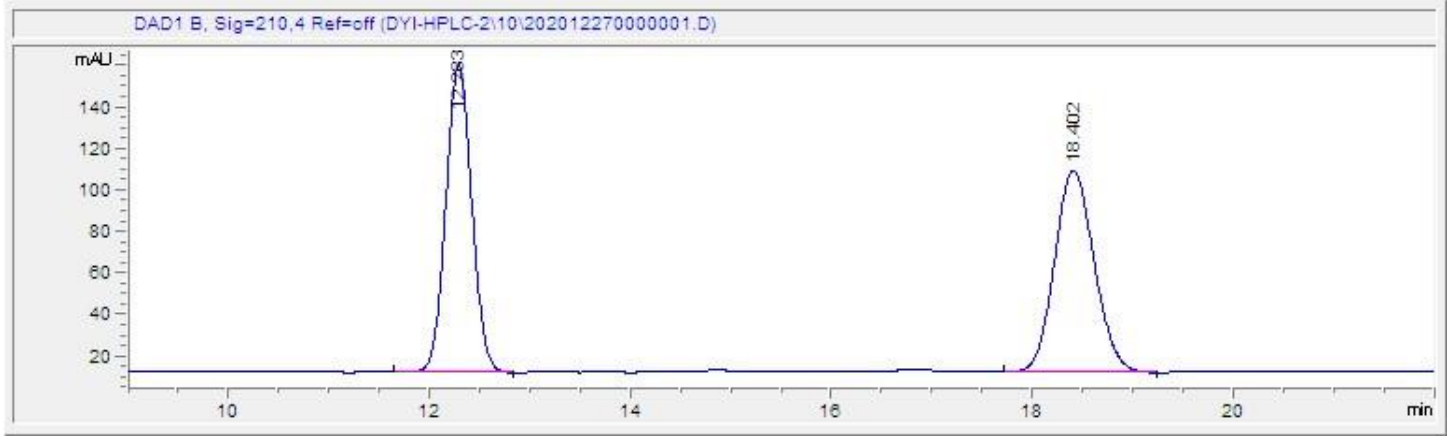

\begin{tabular}{|c|c|c|c|c|c|c|} 
\# & \multicolumn{1}{c}{ Time } & Area & Height & Width & \multicolumn{1}{c|}{ Area\% } & Symmetry \\
\hline 1 & 12.283 & 2663.5 & 148.2 & 0.2796 & 50.017 & 0.929 \\
\hline 2 & 18.402 & 2661.7 & 97.2 & 0.4276 & 49.983 & 0.863 \\
\hline
\end{tabular}


<smiles>C=C[C@H](C1CCCCC1)C(C(=O)OCc1ccccc1)C(=O)OCc1ccccc1</smiles>

$3 g$

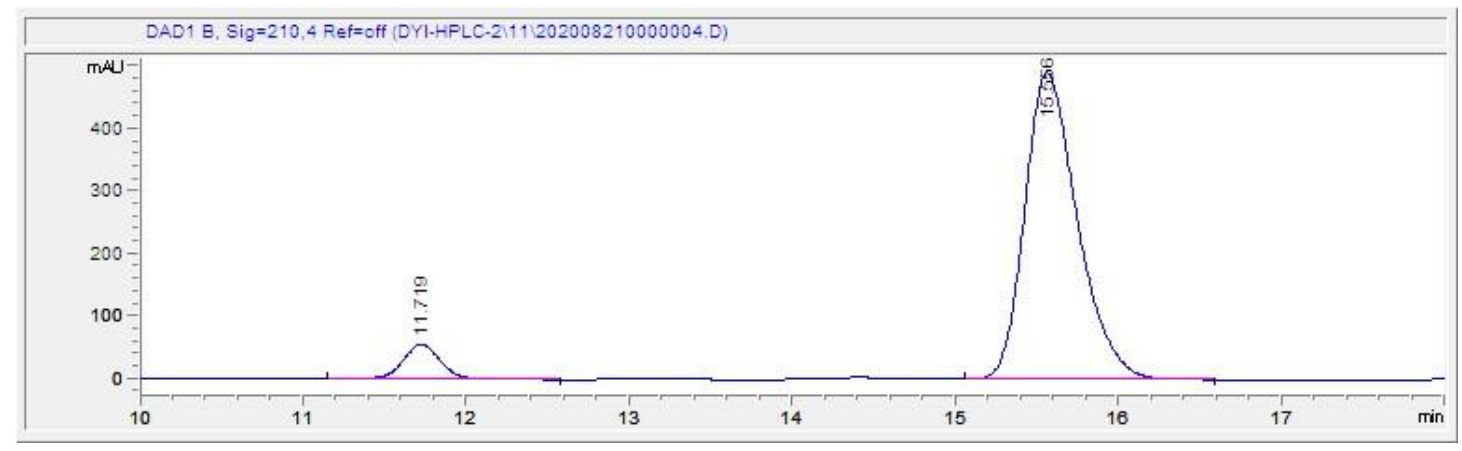

\begin{tabular}{|c|c|c|c|c|c|c|}
\hline$\#$ & Time & Area & Height & Width & Area\% & Symmetry \\
\hline 1 & 11.719 & 877.5 & 55.6 & 0.2422 & 7.402 & 0.948 \\
\hline 2 & 15.556 & 10977.7 & 490.2 & 0.3433 & 92.598 & 0.699 \\
\hline
\end{tabular}

\section{Racemic:}

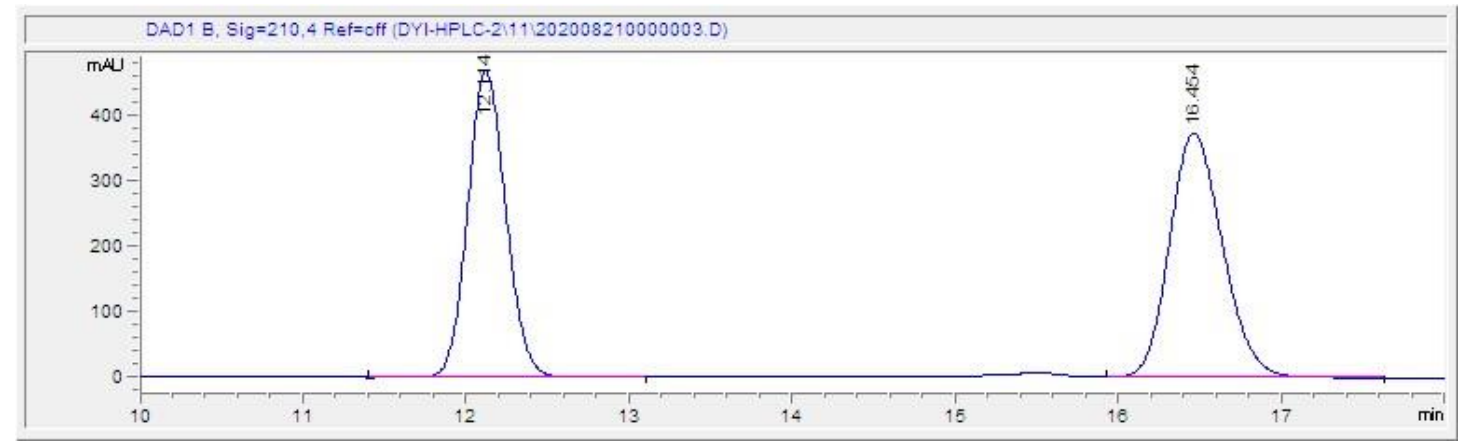

\begin{tabular}{|c|c|c|c|c|c|c|}
\multicolumn{1}{|c}{ \# } & \multicolumn{1}{c}{ Time } & Area & Height & Width & \multicolumn{1}{c}{ Area\% } & Symmetry \\
\hline 1 & 12.114 & 7618.5 & 469.8 & 0.2512 & 47.478 & 0.869 \\
\hline 2 & 16.454 & 8427.9 & 374 & 0.3489 & 52.522 & 0.795 \\
\hline
\end{tabular}


<smiles>C=C[C@H](COC(C)(C)C)C(C(C)=O)C(OC)OC</smiles>

3h

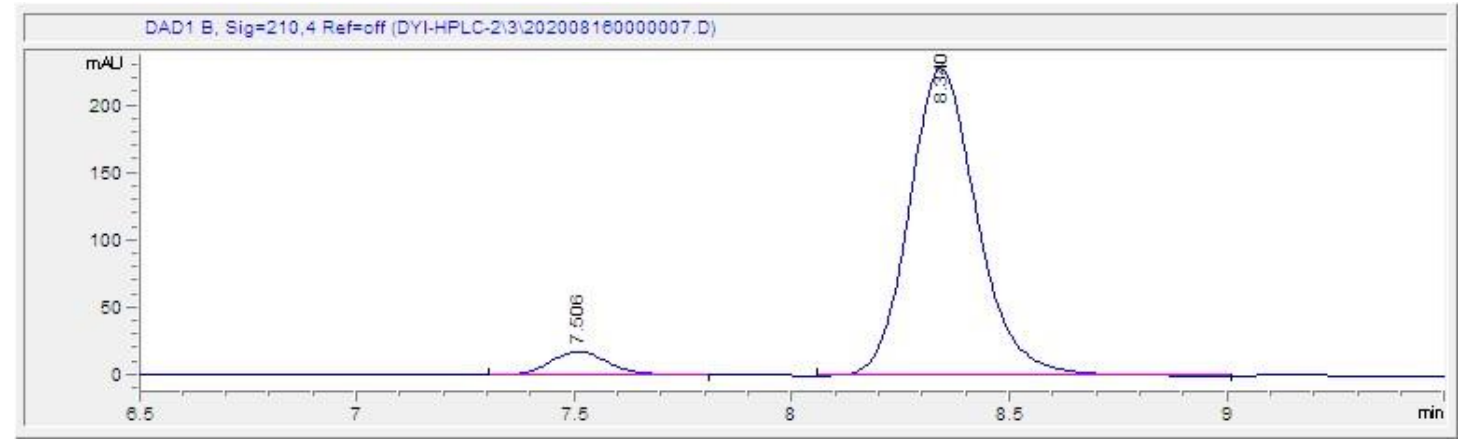

\begin{tabular}{|c|c|c|c|c|c|c|}
\hline$\#$ & Time & Area & Height & Width & Area\% & Symmetry \\
\hline 1 & 7.506 & 159.5 & 17.3 & 0.1416 & 6.056 & 0.87 \\
\hline 2 & 8.34 & 2473.9 & 228.1 & 0.1665 & 93.944 & 0.817 \\
\hline
\end{tabular}

\section{Racemic:}

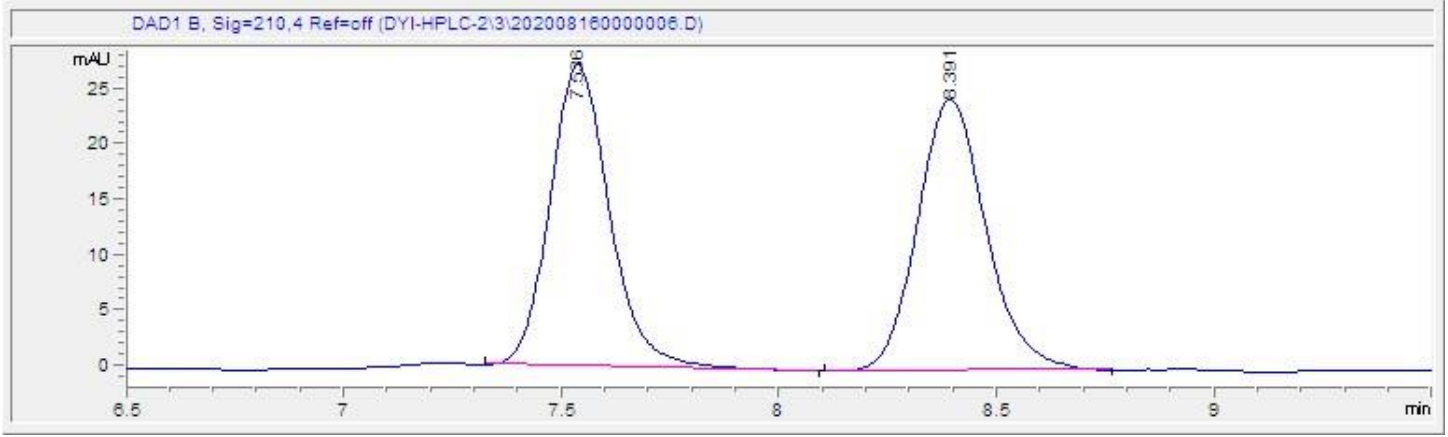

\begin{tabular}{|c|c|c|c|c|c|c|} 
\# & \multicolumn{1}{c}{ Time } & Area & Height & Width & \multicolumn{1}{c}{ Area\% } & Symmetry \\
\hline 1 & 7.536 & 259.6 & 27.2 & 0.1472 & 49.154 & 0.834 \\
\hline 2 & 8.391 & 268.5 & 24.5 & 0.1676 & 50.846 & 0.851 \\
\hline
\end{tabular}


<smiles>C=CC(C(OC)OC)[C@@H](C=C)CCCCOC(C)(C)C</smiles>

$3 \mathbf{i}$

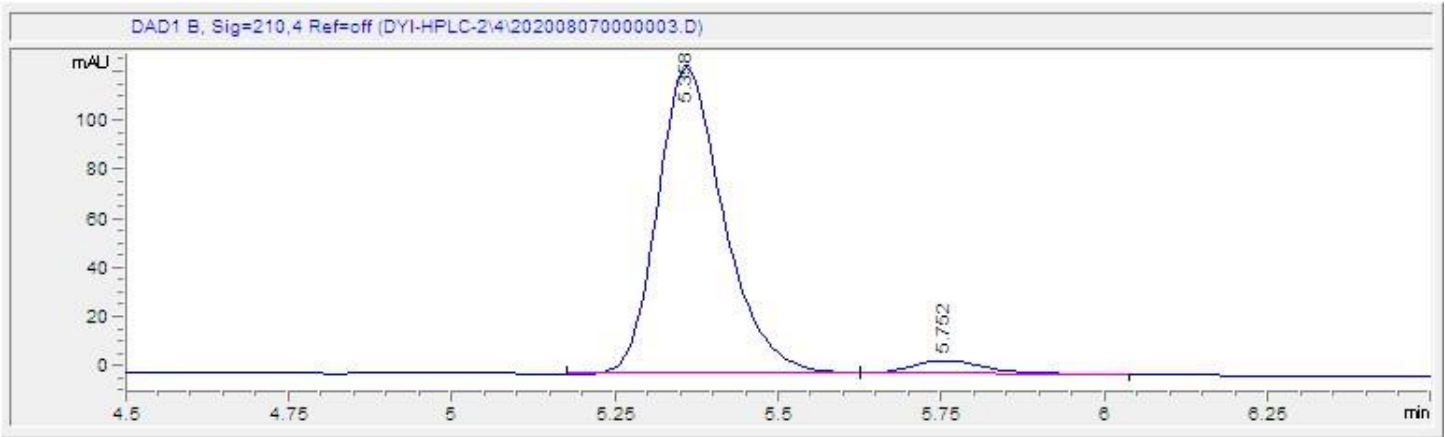

\begin{tabular}{|c|c|c|c|c|c|c|}
\hline$\#$ & Time & Area & Height & Width & Area\% & Symmetry \\
\hline 1 & 5.358 & 879.4 & 124.7 & 0.1083 & 95.560 & 0.735 \\
\hline 2 & 5.752 & 40.9 & 5.3 & 0.116 & 4.440 & 0.679 \\
\hline
\end{tabular}

\section{Racemic:}

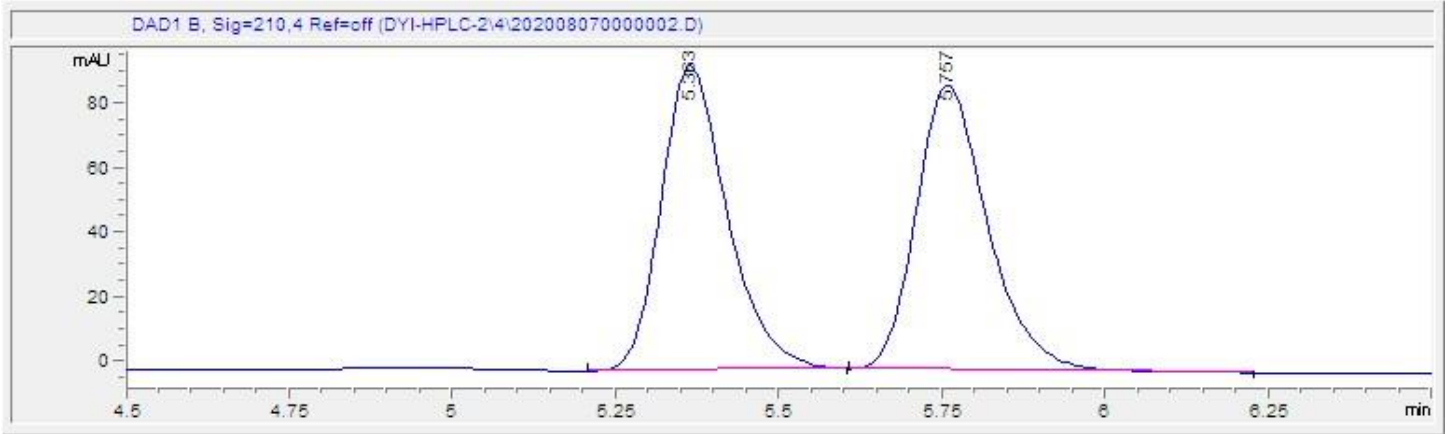

\begin{tabular}{|c|c|c|c|c|c|c|}
\multicolumn{1}{l}{ \# } & Time & Area & Height & Width & \multicolumn{1}{c}{ Area\% } & Symmetry \\
\hline 1 & 5.363 & 665.4 & 94.6 & 0.1061 & 49.710 & 0.739 \\
\hline 2 & 5.757 & 673.2 & 88.4 & 0.1168 & 50.290 & 0.734 \\
\hline
\end{tabular}


<smiles>C=CC(C(OC)OC)[C@H](C=C)CCCCCOC(C)(C)C</smiles>

3j

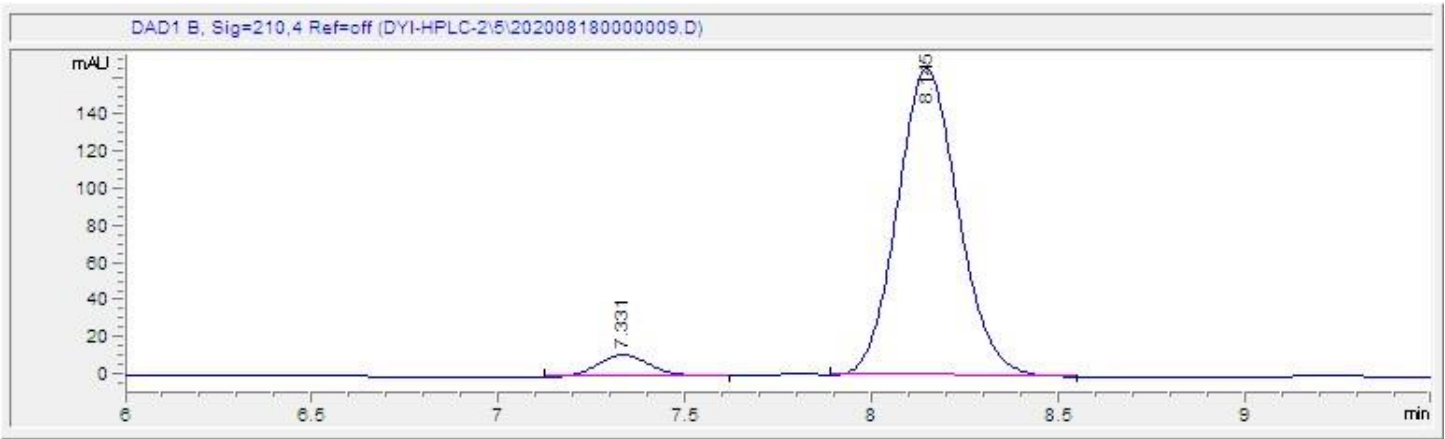

\begin{tabular}{|c|c|c|c|c|c|c|}
\hline \# & Time & Area & Height & Width & Area\% & Symmetry \\
\hline 1 & 7.331 & 115.6 & 11.6 & 0.1542 & 5.790 & 0.87 \\
\hline 2 & 8.145 & 1880.5 & 165.5 & 0.1765 & 94.210 & 0.879 \\
\hline
\end{tabular}

\section{Racemic:}

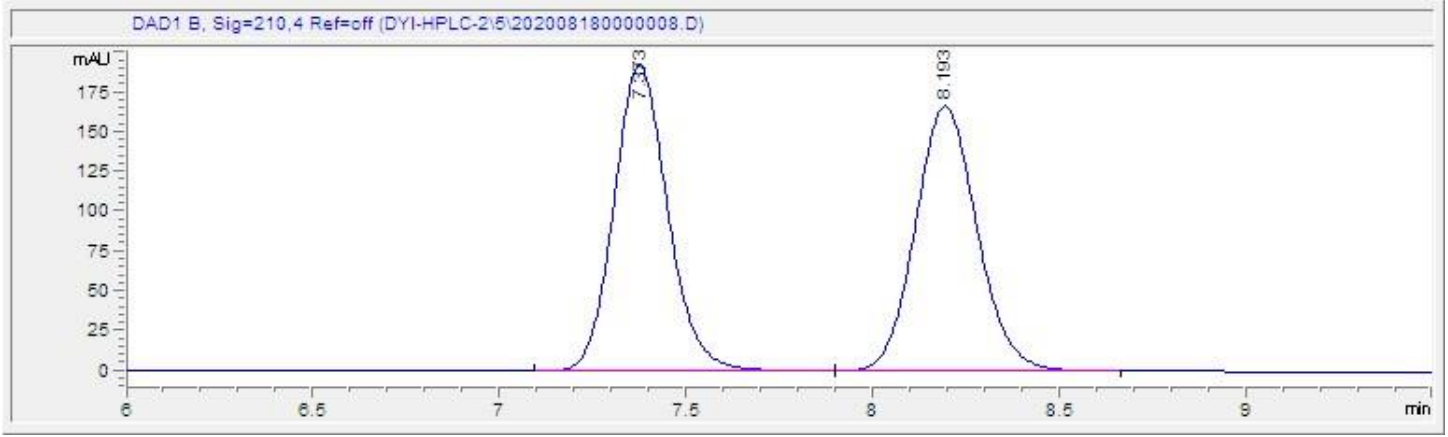

\begin{tabular}{|c|c|c|c|c|c|c|}
\multicolumn{1}{l}{ \# } & Time & \multicolumn{1}{c}{ Area } & Height & Width & \multicolumn{1}{c}{ Area\% } & Symmetry \\
\hline 1 & 7.373 & 1927.6 & 192.7 & 0.1547 & 50.223 & 0.862 \\
\hline 2 & 8.193 & 1910.5 & 166.6 & 0.1778 & 49.777 & 0.887 \\
\hline
\end{tabular}


<smiles>C=C[C@H](CCCCCNC(C)(C)C)C(C(C)=O)C(C)=O</smiles>

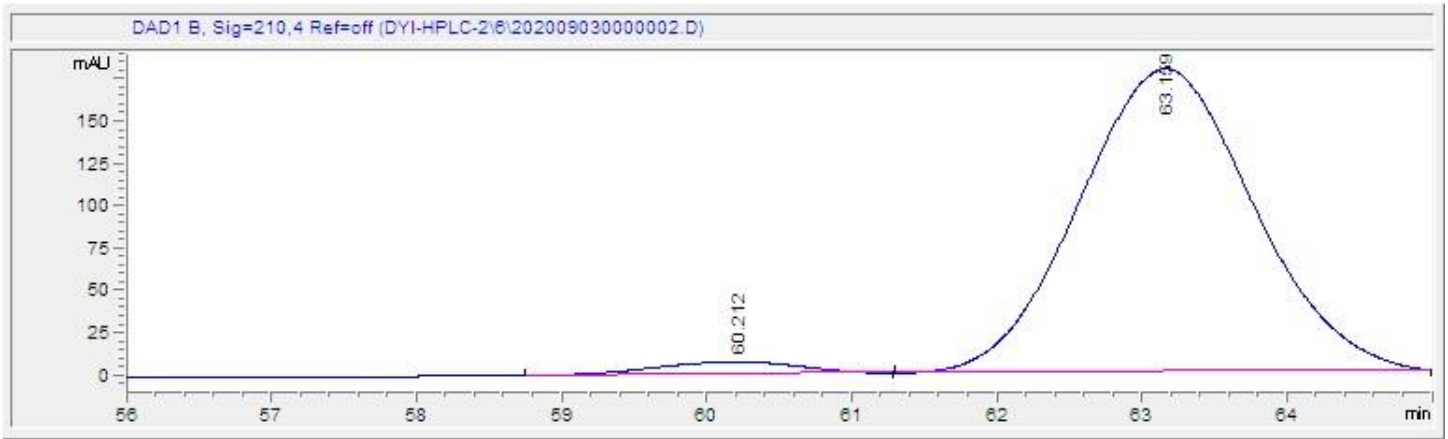

\begin{tabular}{c|c|c|c|c|c|c|}
\multicolumn{1}{l}{ \# } & \multicolumn{1}{c}{ Time } & Area & Height & Width & \multicolumn{1}{c|}{ Area\% } & \multicolumn{1}{c|}{ Symmetry } \\
\hline 1 & 60.212 & 522.4 & 7.5 & 0.8399 & 3.373 & 1.338 \\
\hline 2 & 63.159 & 14964.9 & 179.1 & 1.2906 & 96.627 & 0.957 \\
\hline
\end{tabular}

\section{Racemic:}

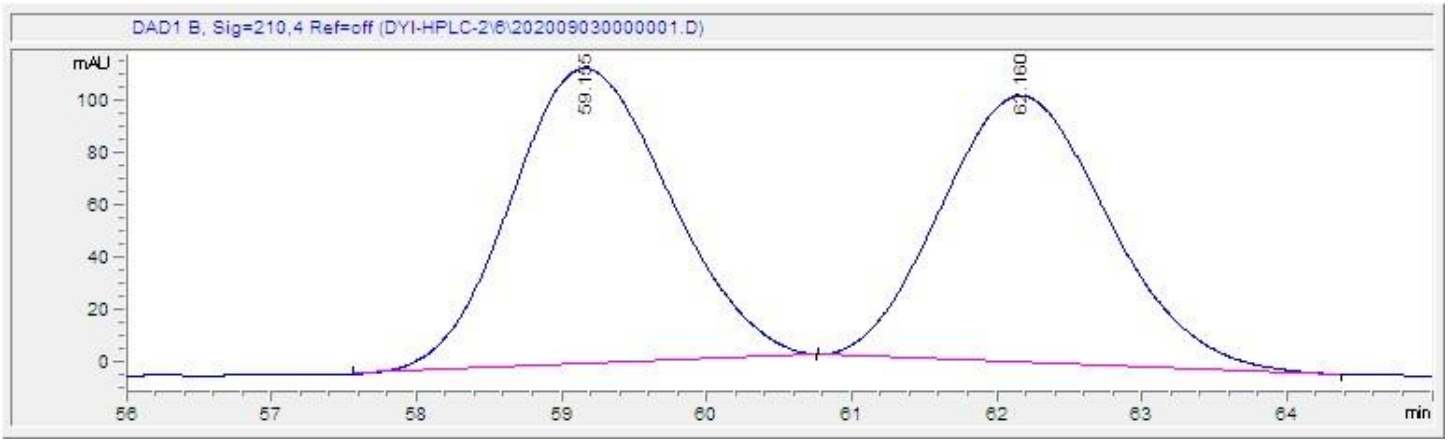

\begin{tabular}{l|c|c|c|c|c|c|} 
\# & \multicolumn{1}{c}{ Time } & Area & Height & \multicolumn{1}{c}{ Width } & \multicolumn{1}{c}{ Area\% } & Symmetry \\
\hline 1 & 59.155 & 8606.8 & 113.5 & 1.1726 & 51.232 & 0.859 \\
\hline 2 & 62.16 & 8192.8 & 102.4 & 1.2658 & 48.768 & 0.866 \\
\hline
\end{tabular}


<smiles>C=CCCC(C=C)C(C(=O)OCc1ccccc1)C(=O)OCc1ccccc1</smiles>

3I

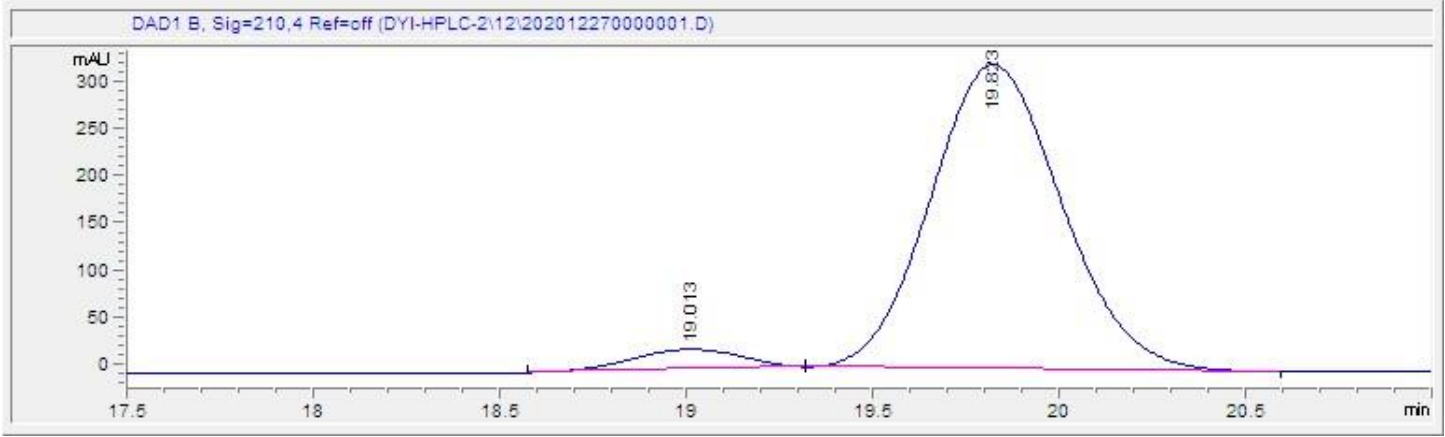

\begin{tabular}{|c|c|c|c|c|c|c|}
\hline$\#$ & Time & Area & Height & Width & Area\% & Symmetry \\
\hline 1 & 19.013 & 420.4 & 20.9 & 0.3204 & 5.084 & 1.174 \\
\hline 2 & 19.823 & 7848 & 321.5 & 0.3808 & 94.916 & 0.877 \\
\hline
\end{tabular}

\section{Racemic:}

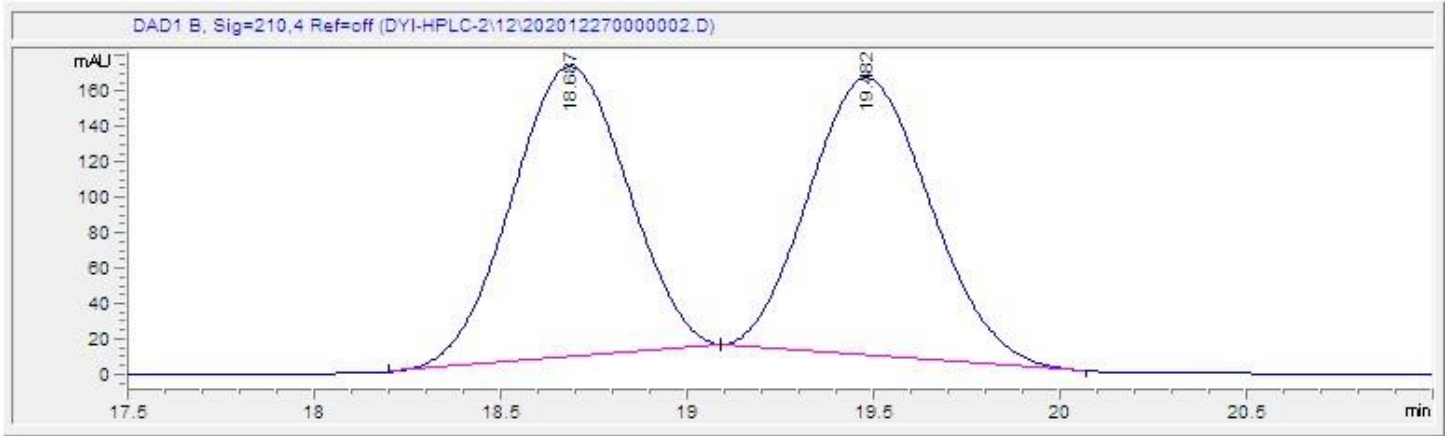

\begin{tabular}{|c|c|c|c|c|c|c|} 
\# & Time & Area & Height & Width & \multicolumn{1}{c}{ Area\% } & Symmetry \\
\hline 1 & 18.687 & 3543 & 164.3 & 0.3396 & 49.991 & 1.005 \\
\hline 2 & 19.482 & 3544.2 & 156.7 & 0.3559 & 50.009 & 0.863 \\
\hline
\end{tabular}


<smiles>C=C[C@H](C[C@@H](C)CCC=C(C)C)C(C(=O)OCc1ccccc1)C(=O)OCc1ccccc1</smiles>

$3 m$

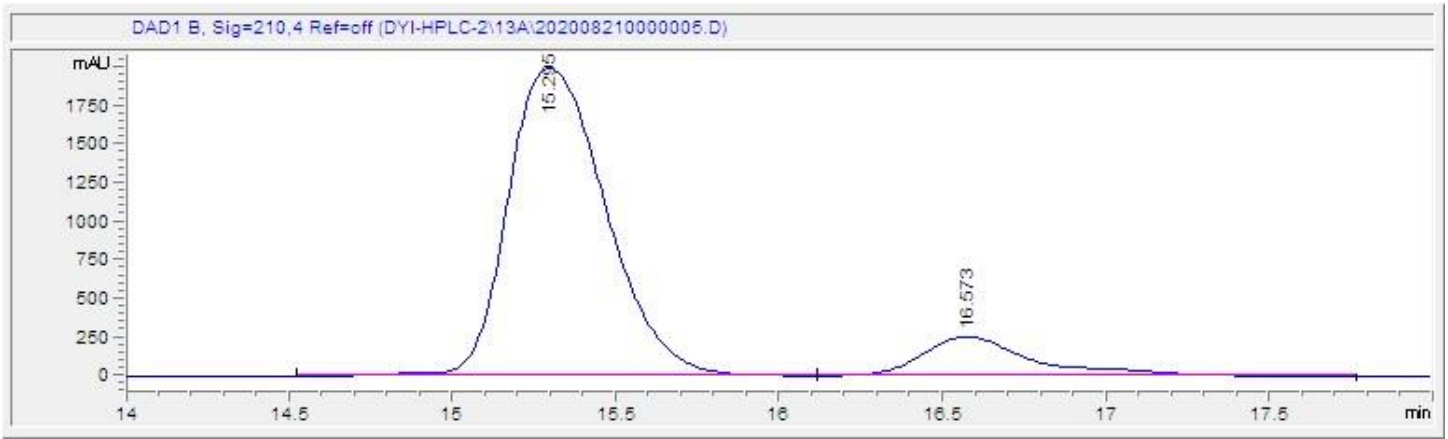

\begin{tabular}{|c|c|c|c|c|c|c|}
\hline$\#$ & Time & Area & Height & Width & Area\% & Symmetry \\
\hline 1 & 15.295 & 41581.2 & 1990.9 & 0.3277 & 87.572 & 0.704 \\
\hline 2 & 16.573 & 5901.1 & 252.4 & 0.3467 & 12.428 & 0.611 \\
\hline
\end{tabular}

Mixture of Diastereoisomers:

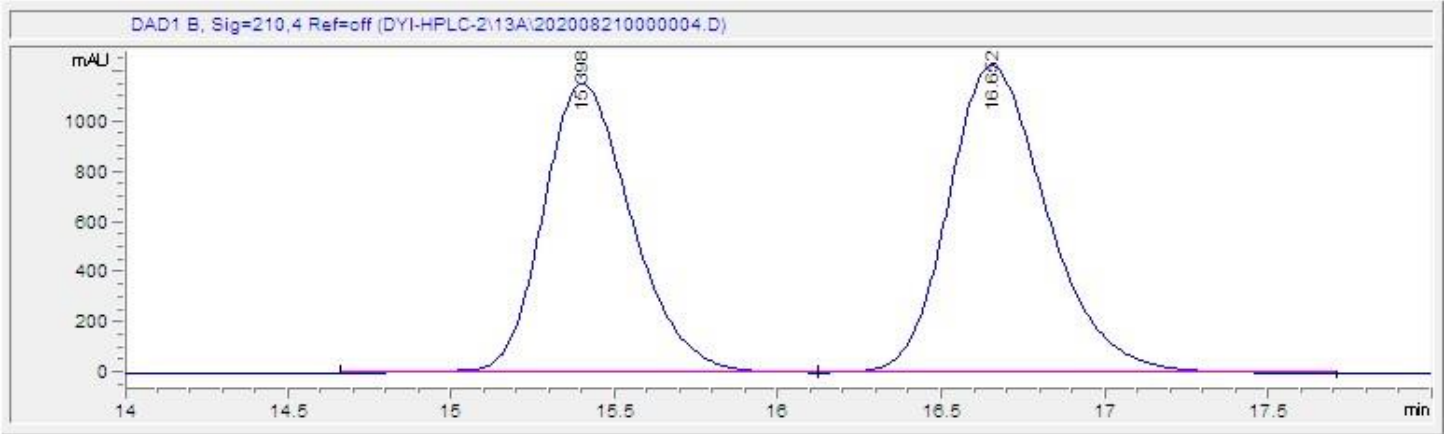

\begin{tabular}{|c|c|c|c|c|c|c|}
\multicolumn{1}{c}{ \# } & \multicolumn{1}{c}{ Time } & \multicolumn{1}{c}{ Area } & Height & width & \multicolumn{1}{c}{ Area\% } & Symmetry \\
\hline 1 & 15.398 & 21738.2 & 1150.2 & 0.2925 & 45.770 & 0.765 \\
\hline 2 & 16.652 & 25756.4 & 1222.6 & 0.3258 & 54.230 & 0.75 \\
\hline
\end{tabular}


<smiles>C=CC(C[C@@H](C)CCC=C(C)C)C(C(=O)OCc1ccccc1)C(=O)OCc1ccccc1</smiles>

3n

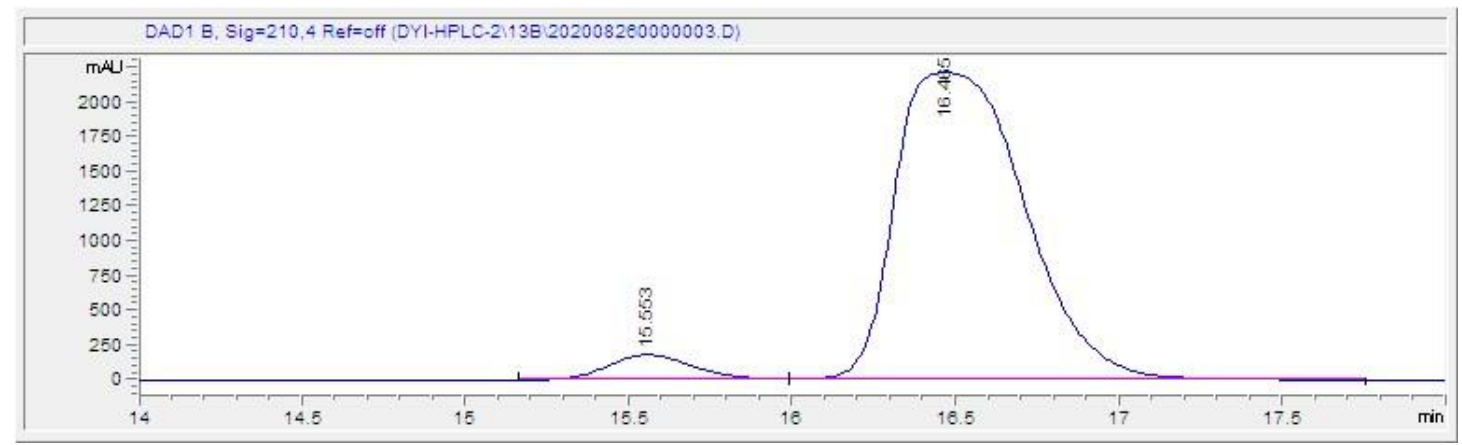

\begin{tabular}{c|c|c|c|c|c|c|}
\multicolumn{1}{c}{ \# } & Time & Area & Height & width & \multicolumn{1}{c|}{ Area\% } & \multicolumn{1}{c|}{ Symmetry } \\
\hline 1 & 15.553 & 3212.7 & 179.4 & 0.277 & 5.026 & 0.835 \\
\hline 2 & 16.465 & 60713.5 & 2211.5 & 0.4363 & 94.974 & 0.587 \\
\hline
\end{tabular}

\section{Mixture of Diastereoisomers:}

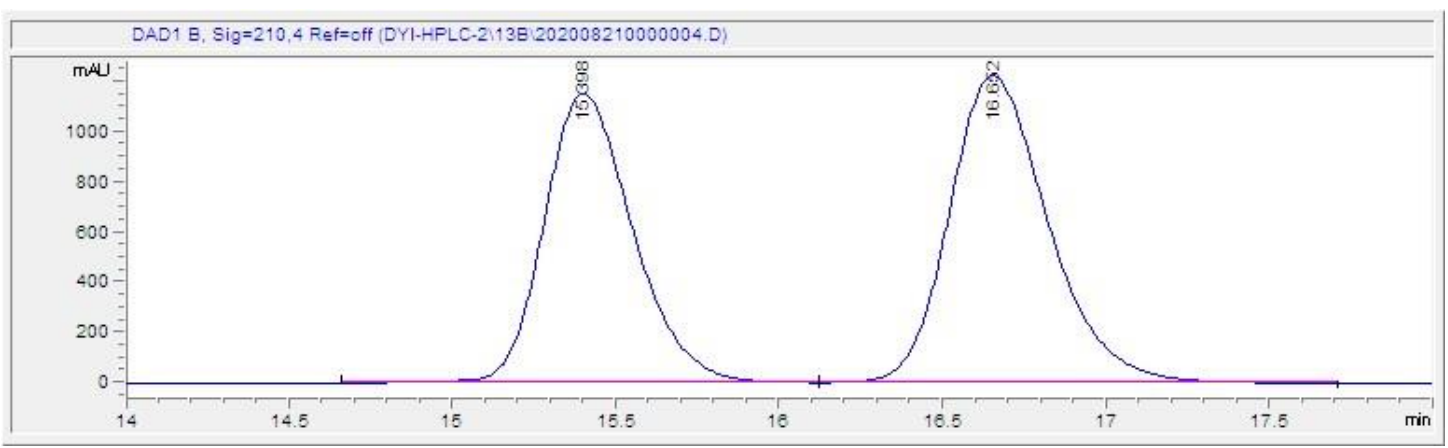

\begin{tabular}{|c|c|c|c|c|c|c|}
\hline$\#$ & Time & Area & Height & Width & Area\% & Symmetry \\
\hline 1 & 15.398 & 21738.2 & 1150.2 & 0.2925 & 45.770 & \begin{tabular}{|l}
0.765 \\
\end{tabular} \\
\hline 2 & 16.652 & 25756.4 & 1222.6 & 0.3258 & 54.230 & 0.75 \\
\hline
\end{tabular}




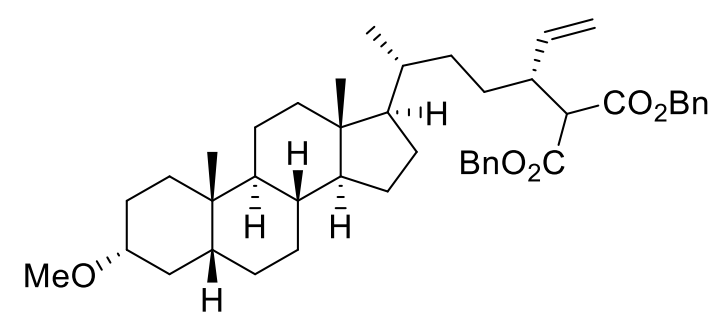

30

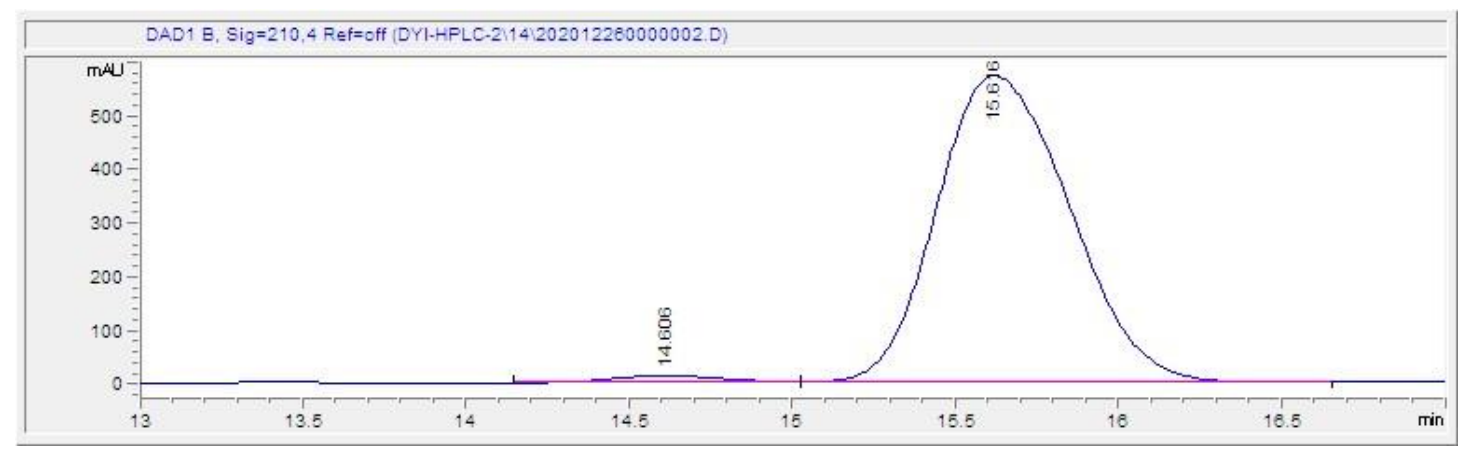

\begin{tabular}{|c|c|c|c|c|c|c|}
\multicolumn{1}{c}{ \# } & \multicolumn{1}{c}{ Time } & Area & Height & Width & \multicolumn{1}{c|}{ Area\% } & \multicolumn{1}{c|}{ Symmetry } \\
\hline 1 & 14.606 & 266.1 & 12.1 & 0.3439 & 1.626 & 0.956 \\
\hline 2 & 15.616 & 16096.9 & 570.2 & 0.4554 & 98.374 & 0.743 \\
\hline
\end{tabular}

\section{Mixture of Diastereoisomers:}

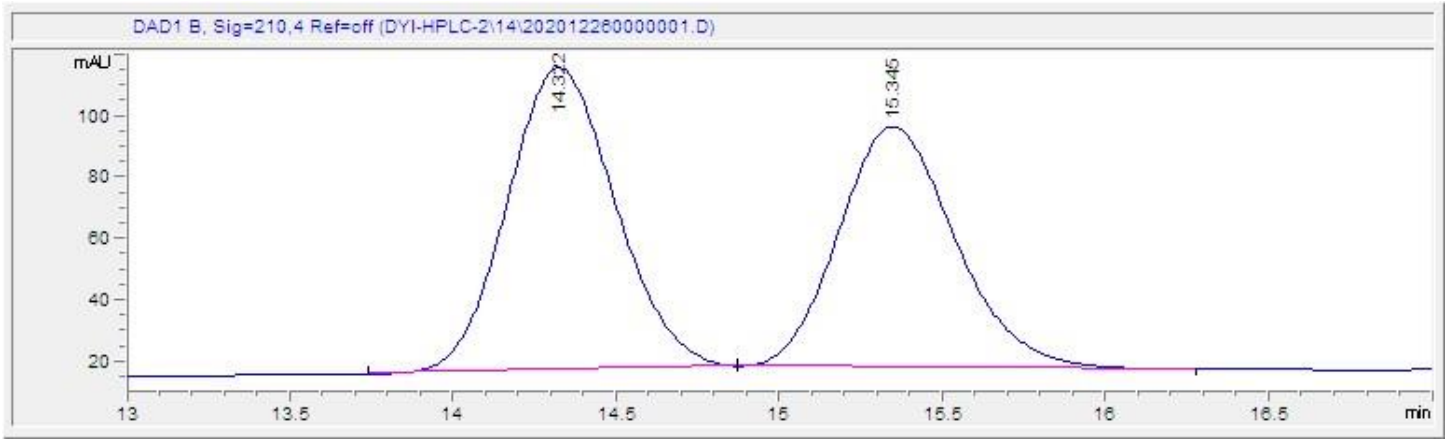

\begin{tabular}{r|c|c|c|c|c|c|}
\multicolumn{1}{l}{ \# } & \multicolumn{1}{c}{ Time } & Area & Height & Width & \multicolumn{1}{c}{ Area\% } & \multicolumn{1}{c}{ Symmetry } \\
\hline 1 & 14.322 & 2262.6 & 98.4 & 0.3582 & 53.734 & 0.89 \\
\hline 2 & 15.345 & 1948.2 & 78.5 & 0.3857 & 46.266 & 0.822 \\
\hline
\end{tabular}


<smiles>C=C[C@H](CCc1ccccc1)[C@H](C(=O)CCC)C(=O)OCC</smiles>

$3 p$

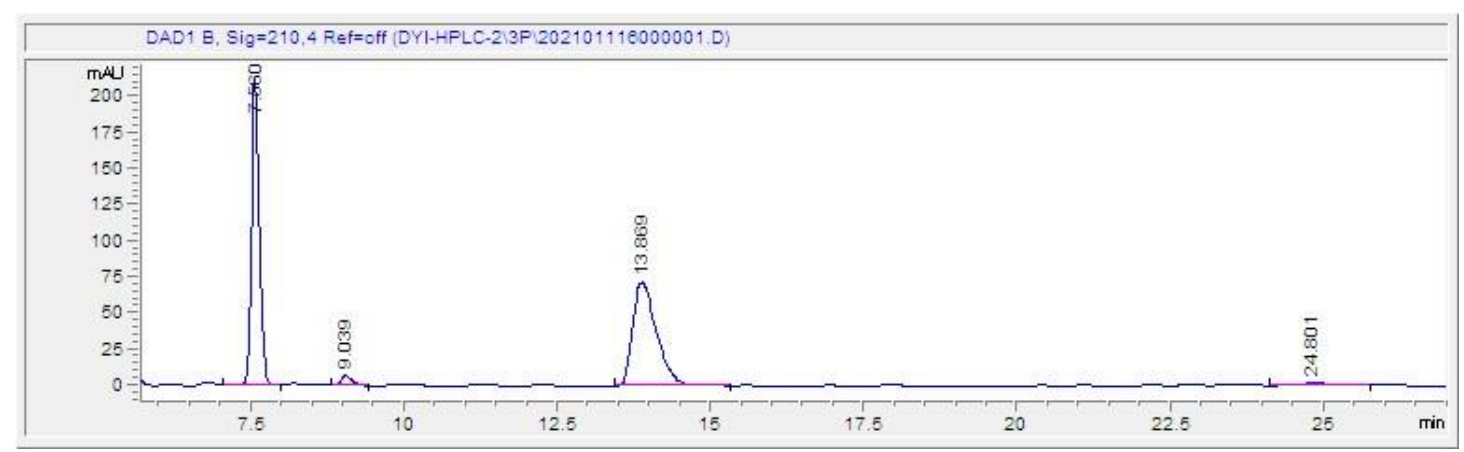

\begin{tabular}{|c|c|c|c|c|c|c|}
\hline$\#$ & Time & Area & Height & Width & Area\% & Symmetry \\
\hline 1 & 7.56 & 1908 & 212.3 & 0.1388 & 47.896 & 0.825 \\
\hline 2 & 9.039 & 84 & 7 & 0.1825 & 2.109 & 0.728 \\
\hline 3 & 13.869 & 1921.3 & 71.3 & 0.4143 & 48.232 & 0.589 \\
\hline 4 & 24.801 & 70.2 & 1.4 & 0.6119 & 1.763 & 0.713 \\
\hline
\end{tabular}

\section{Racemic:}

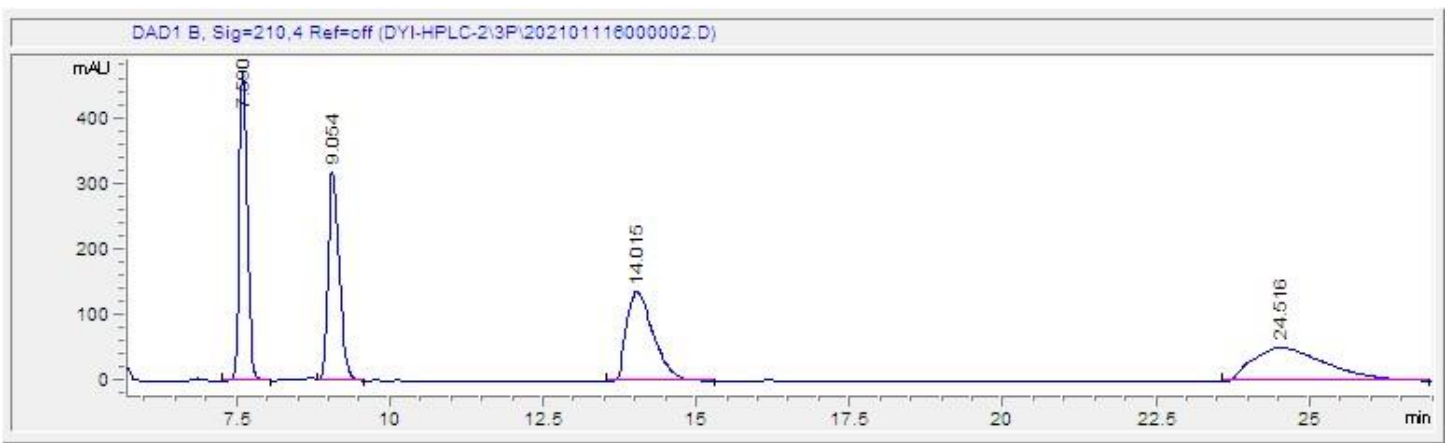

\begin{tabular}{|c|c|c|c|c|c|c|}
\hline \# & Time & Area & Height & Width & Area\% & Symmetry \\
\hline 1 & 7.59 & 4230.7 & 467.6 & 0.1396 & 25.599 & 0.818 \\
\hline 2 & 9.054 & 4037.9 & 317.5 & 0.1964 & 24.432 & 0.674 \\
\hline 3 & 14.015 & 4090.2 & 134.6 & 0.4806 & 24.749 & 0.582 \\
\hline 4 & 24.516 & 4168.1 & 50.5 & 1.1566 & 25.220 & 0.596 \\
\hline
\end{tabular}




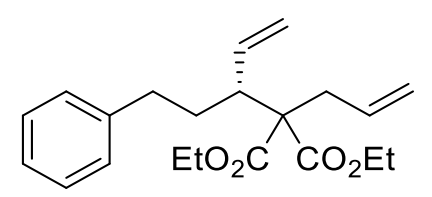

$3 q$

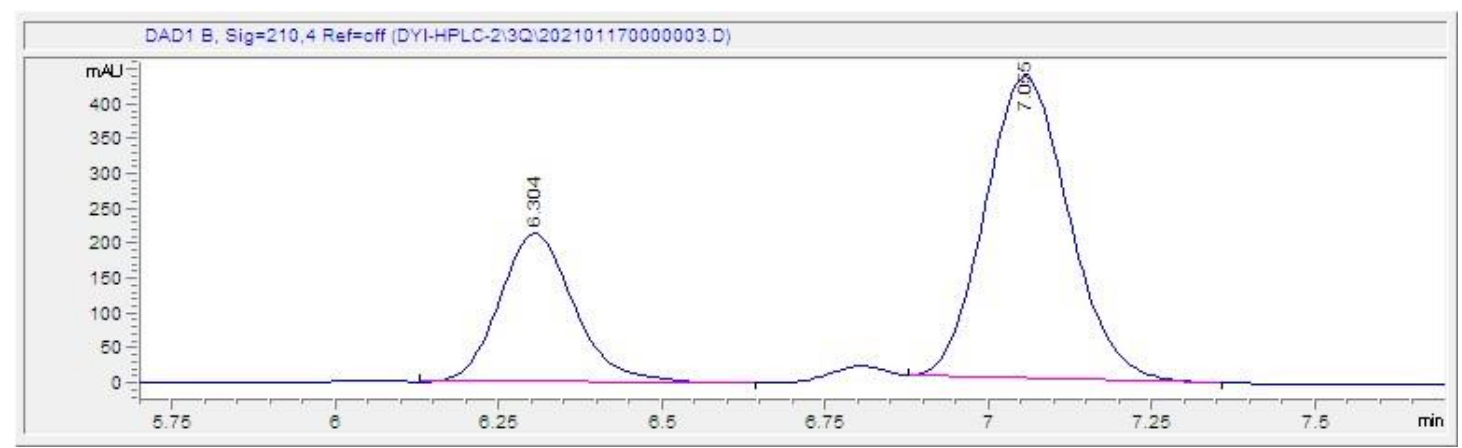

\begin{tabular}{|c|c|c|c|c|c|c|}
\hline$\#$ & Time & Area & Height & width & Area\% & Symmetry \\
\hline 1 & 6.304 & 1704.6 & 213.9 & 0.123 & 30.626 & \begin{tabular}{|l|}
0.844 \\
\end{tabular} \\
\hline 2 & 7.055 & 3861.2 & 433 & 0.14 & 69.374 & 0.875 \\
\hline
\end{tabular}

\section{Racemic:}

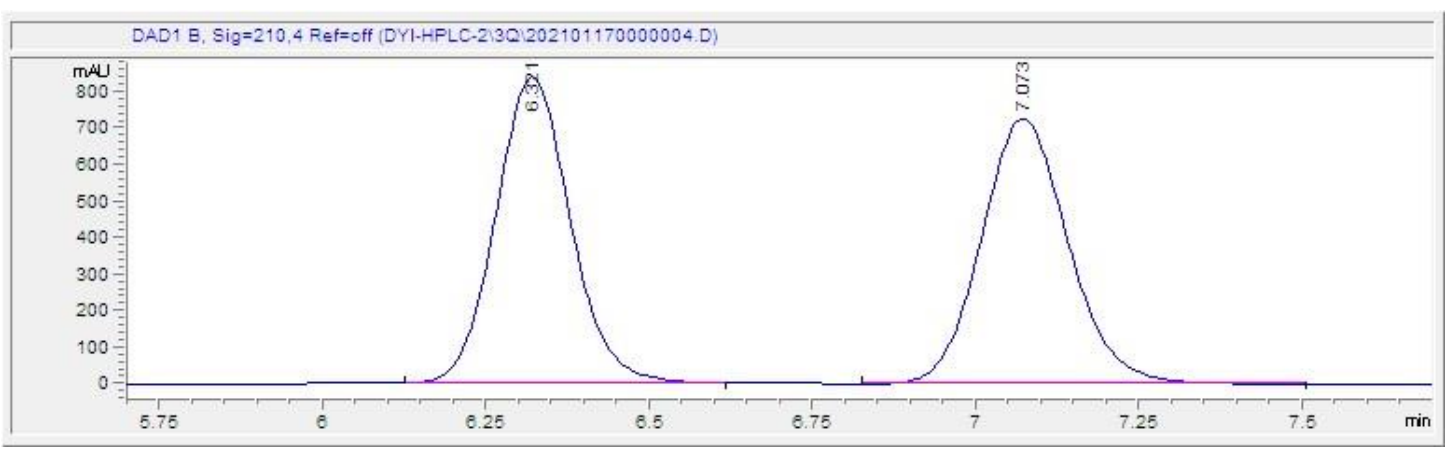

\begin{tabular}{|c|c|c|c|c|c|c|}
\hline \# & Time & Area & Height & Width & Area\% & Symmetry \\
\hline 1 & 6.321 & 6636.5 & 837.8 & 0.1224 & 49.778 & 0.914 \\
\hline 2 & 7.073 & 6695.7 & 726 & 0.1436 & 50.222 & 0.885 \\
\hline
\end{tabular}




\section{Part of the original HRMS analysis reports}

Qualitative Analysis Report

\begin{tabular}{|c|c|c|c|c|}
\hline Data Filename & ap06098.d & & Sample Name & ар06098 \\
\hline Sample Type & Sample & & Position & P1-F3 \\
\hline Instrument Name & Instrument 1 & & User Name & \\
\hline Acq Method & s.m & & Acquired Time & 9/29/2020 10:36:53 AM \\
\hline IRM Calibration Status & Success & & DA Method & Default.m \\
\hline \multicolumn{5}{|l|}{ Comment } \\
\hline Sample Group & & Info. & & \\
\hline $\begin{array}{l}\text { Acquisition SW } \\
\text { Version }\end{array}$ & $\begin{array}{l}\text { es TOF/6500 series } \\
05.01(B 5125,2)\end{array}$ & & & \\
\hline
\end{tabular}

User Spectra
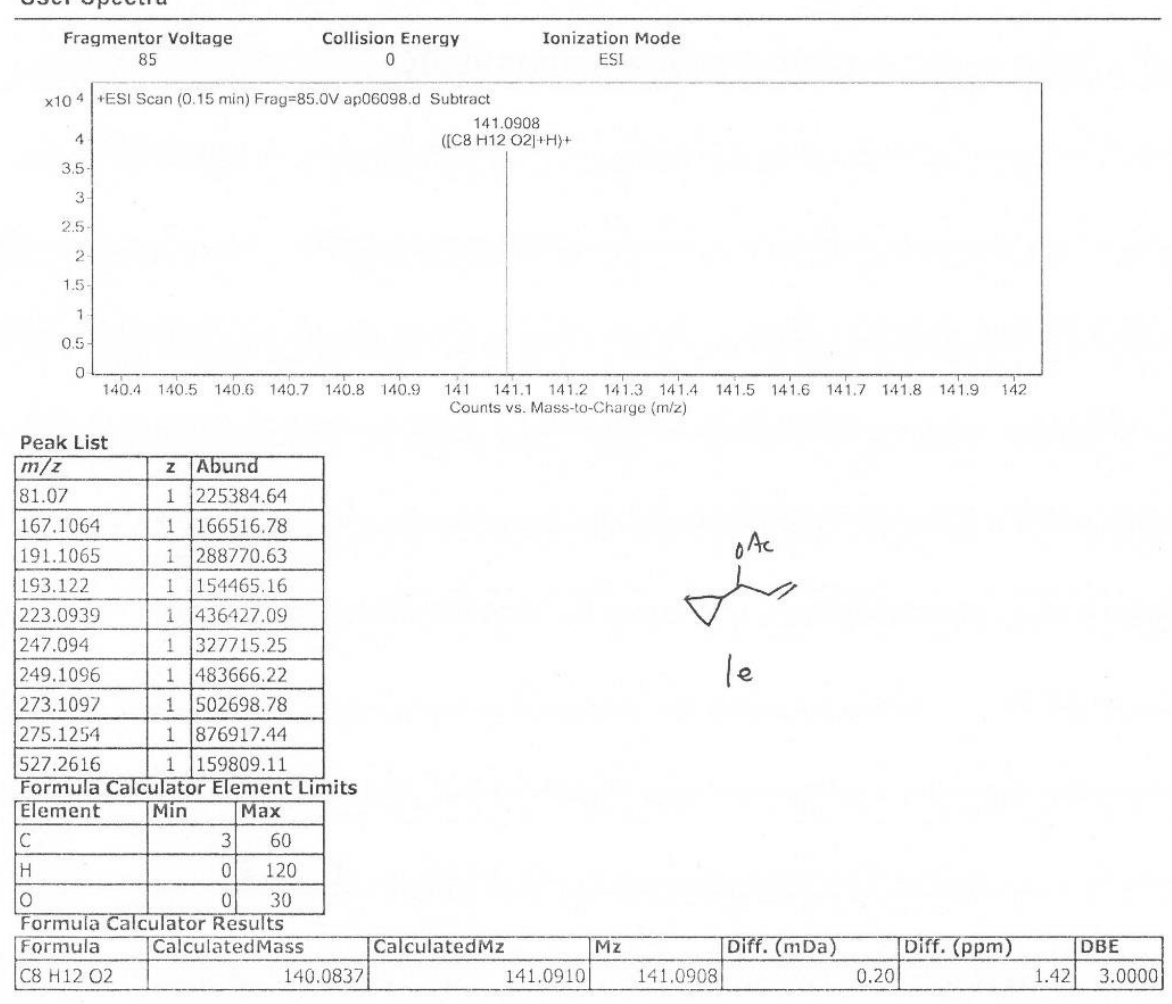

-- End Of Report -.- 
Qualitative Analysis Report

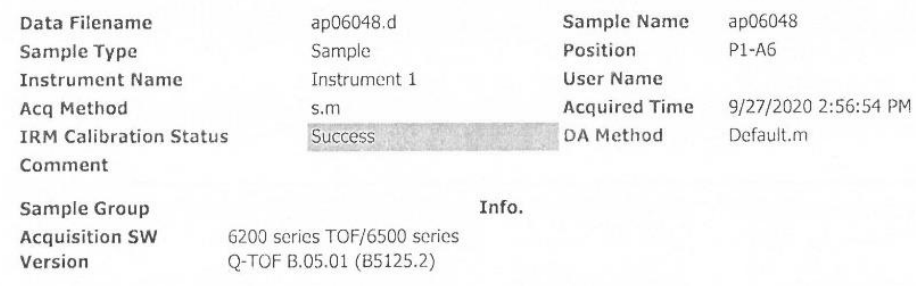

User Spectra
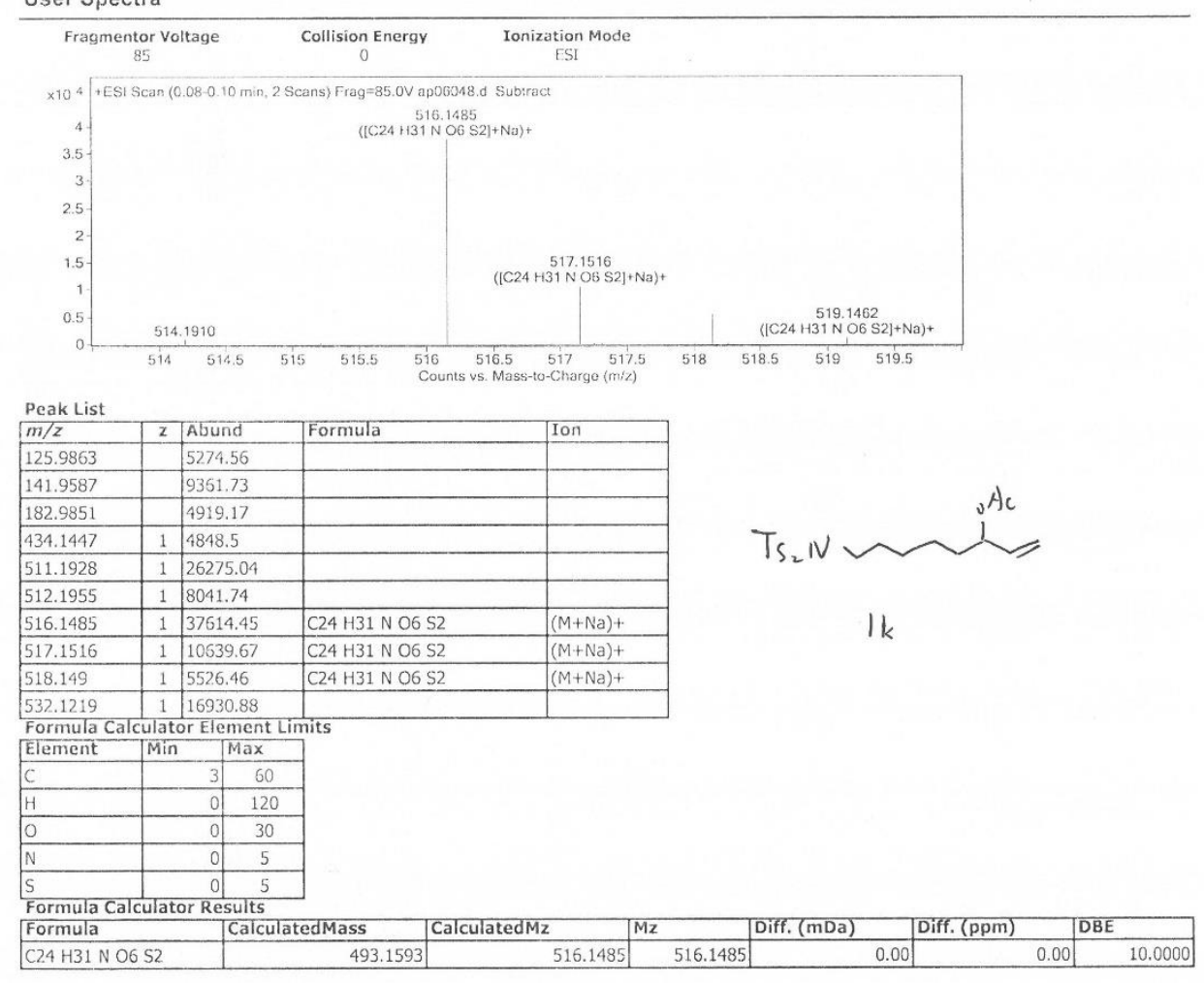

-.. End Of Report ... 
Qualitative Analysis Report

\begin{tabular}{|c|c|c|c|}
\hline Data Filename & ap06022.d & Sample Name & ap06022 \\
\hline Sample Type & Sample & Position & P1-A1 \\
\hline Instrument Name & Instrument 1 & User Name & \\
\hline Acq Method & s.m & Acquired Time & $12 / 25 / 202010: 30: 17$ AM \\
\hline IRM Calibration Status & Success & DA Method & Default.m \\
\hline \multicolumn{4}{|l|}{ Comment } \\
\hline Sample Group & & & \\
\hline $\begin{array}{l}\text { Acquisition SW } \\
\text { Version }\end{array}$ & $\begin{array}{l}\text { ies TOF/6500 series } \\
05.01 \text { (B5125.2) }\end{array}$ & & \\
\hline
\end{tabular}

User Spectra

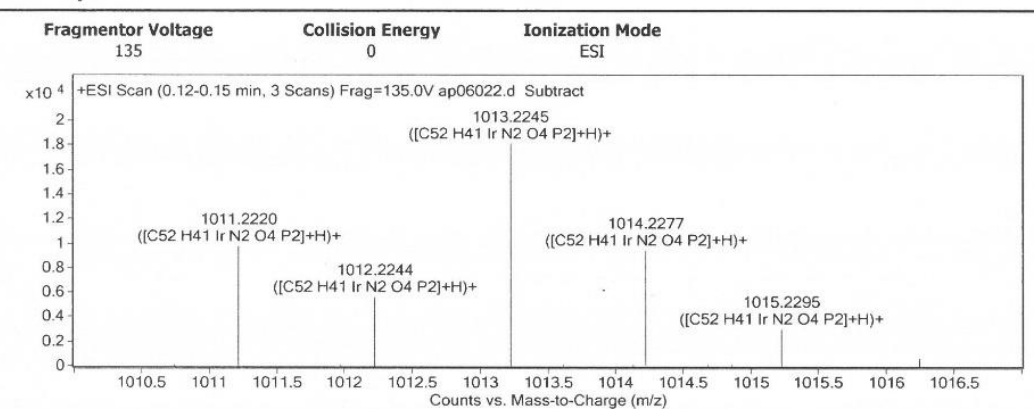

Peak List

\begin{tabular}{|c|c|c|c|c|}
\hline \multicolumn{5}{|l|}{ Peak List } \\
\hline$m / z$ & $z$ & \multicolumn{2}{|c|}{ Abund } & Fo \\
\hline 869.193 & 1 & \multicolumn{2}{|c|}{1863.08} & \\
\hline 1011.222 & 1 & \multicolumn{2}{|c|}{9790.64} & C5 \\
\hline 1012.2244 & 1 & \multicolumn{2}{|c|}{5644.41} & C5 \\
\hline 1013.2245 & 1 & \multicolumn{2}{|c|}{18141.8} & $\mathrm{C5}$ \\
\hline 1014.2277 & 1 & \multicolumn{2}{|c|}{9453.91} & C5 \\
\hline 1015.2295 & 1 & \multicolumn{2}{|c|}{3031.4} & C5 \\
\hline 1033.2024 & 1 & \multicolumn{2}{|c|}{2284.45} & \\
\hline 1034.2051 & 1 & \multicolumn{2}{|c|}{1566.34} & \\
\hline 1035.2068 & 1 & \multicolumn{2}{|c|}{4194.76} & \\
\hline 1036.2092 & 1 & \multicolumn{2}{|c|}{2307.5} & \\
\hline \multirow{2}{*}{\multicolumn{4}{|c|}{$\begin{array}{l}\text { Formula Calculator Element } \\
\begin{array}{l|l|l|}\text { Element } & \text { Min } & \text { Max }\end{array}\end{array}$}} & \\
\hline & & & & \\
\hline C & & 3 & 120 & \\
\hline $\mathrm{H}$ & & 0 & 240 & \\
\hline 0 & & 0 & 30 & \\
\hline $\mathrm{N}$ & & 0 & 5 & \\
\hline $\mathrm{P}$ & & 0 & 5 & \\
\hline Ir & & 1 & 1 & \\
\hline
\end{tabular}

Formula Calculator Results

\begin{tabular}{|l|c|c|r|r|r|}
\hline Formula & CalculatedMass & CalculatedMz & Mz & Diff. (mDa) & Diff. (ppm) \\
\hline C52 H41 Ir N2 O4 P2 & 1010.2148 & 1011.2220 & 1011.2220 & 0.00 & 0.00 \\
\hline
\end{tabular}

...- End Of Report ...

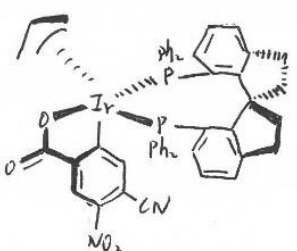

$(R)-I_{r}-S D P$ cat-2 


\section{Qualitative Analysis Report}

\begin{tabular}{|c|c|c|c|c|}
\hline Data Filename & at20142-1.d & & Sample Name & at20142-1 \\
\hline Sample Type & Sample & & Position & P1-A1 \\
\hline Instrument Name & Instrument 1 & & User Name & \\
\hline Acq Method & s.m & & Acquired Time & 9/27/2020 2:51:00 PM \\
\hline IRM Calibration Status & Success & & DA Method & Default.m \\
\hline \multicolumn{5}{|l|}{ Comment } \\
\hline Sample Group & & Info. & & \\
\hline $\begin{array}{l}\text { Acquisition SW } \\
\text { Version }\end{array}$ & $\begin{array}{l}\text { ies TOF/6500 series } \\
05.01(B 5125.2)\end{array}$ & & & \\
\hline
\end{tabular}

User Spectra
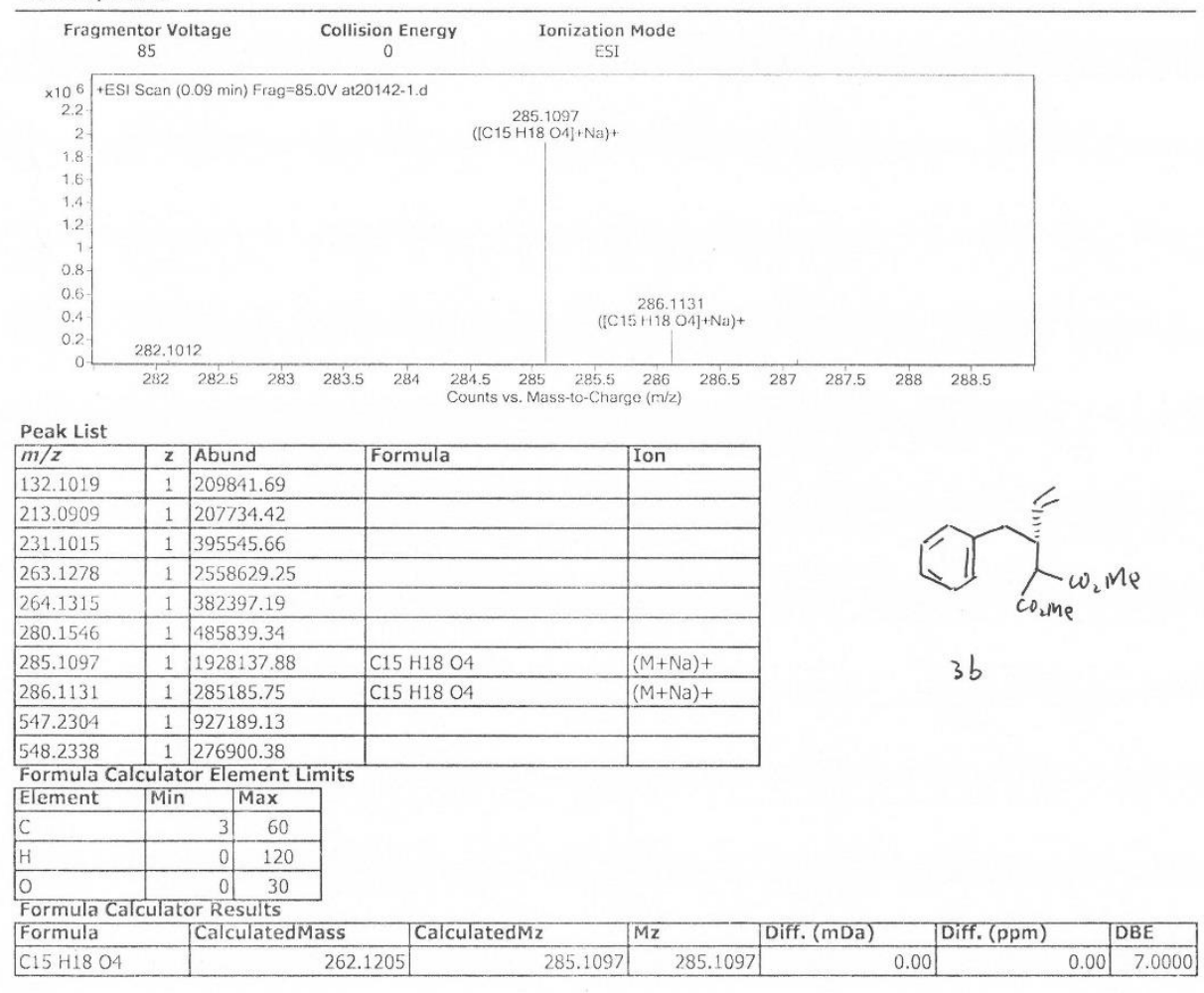

-.- End Of Report -.- 


\section{Qualitative Analysis Report}

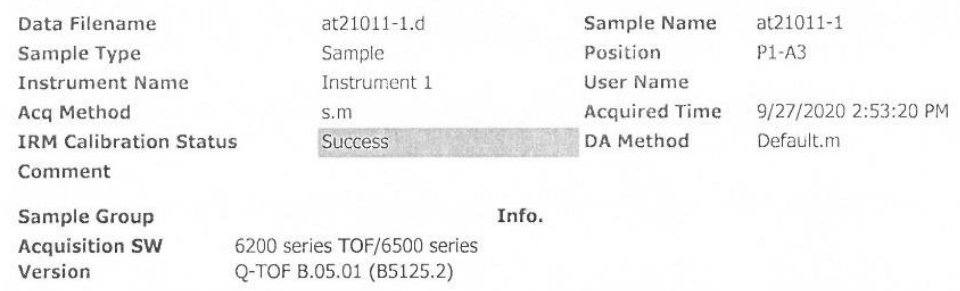

User Spectra
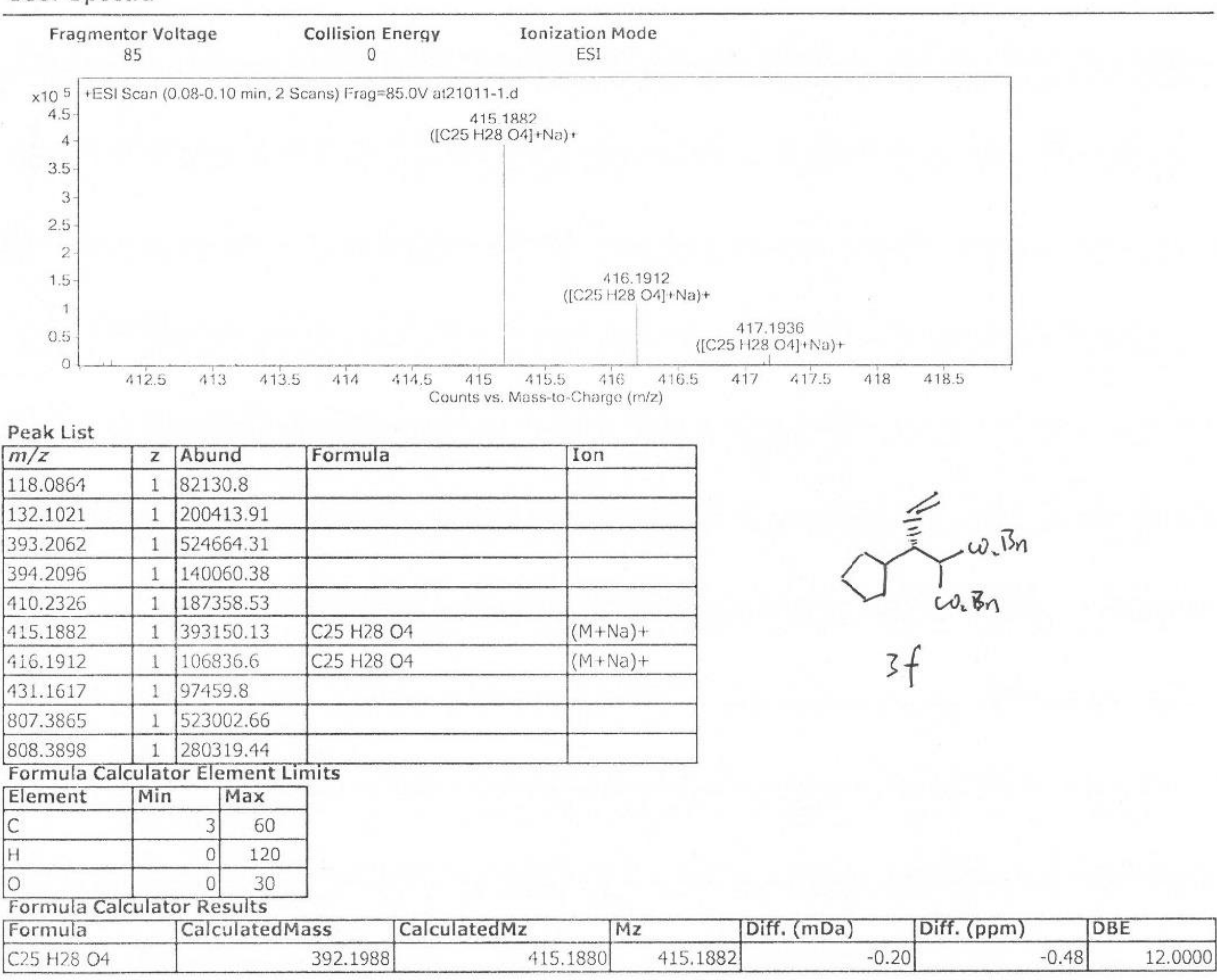

-. End Of Report -.. 
Qualitative Analysis Report

\begin{tabular}{|c|c|c|c|c|}
\hline Data Filename & at21008-1.d & & Sample Name & at21008-1 \\
\hline Sample Type & Sample & & Position & P1-A8 \\
\hline Instrument Name & Instrument 1 & & User Name & \\
\hline Acq Method & s.m & & Acquired Time & 9/24/2020 3:01:49 PM \\
\hline IRM Calibration Status & Success & & DA Method & Default.m \\
\hline \multicolumn{5}{|l|}{ Comment } \\
\hline Sample Group & & Info. & & \\
\hline $\begin{array}{l}\text { Acquisition SW } \\
\text { Version }\end{array}$ & $\begin{array}{l}\text { ies TOF } / 6500 \text { series } \\
.05 .01(B 5125.2)\end{array}$ & & & \\
\hline
\end{tabular}

User Spectra
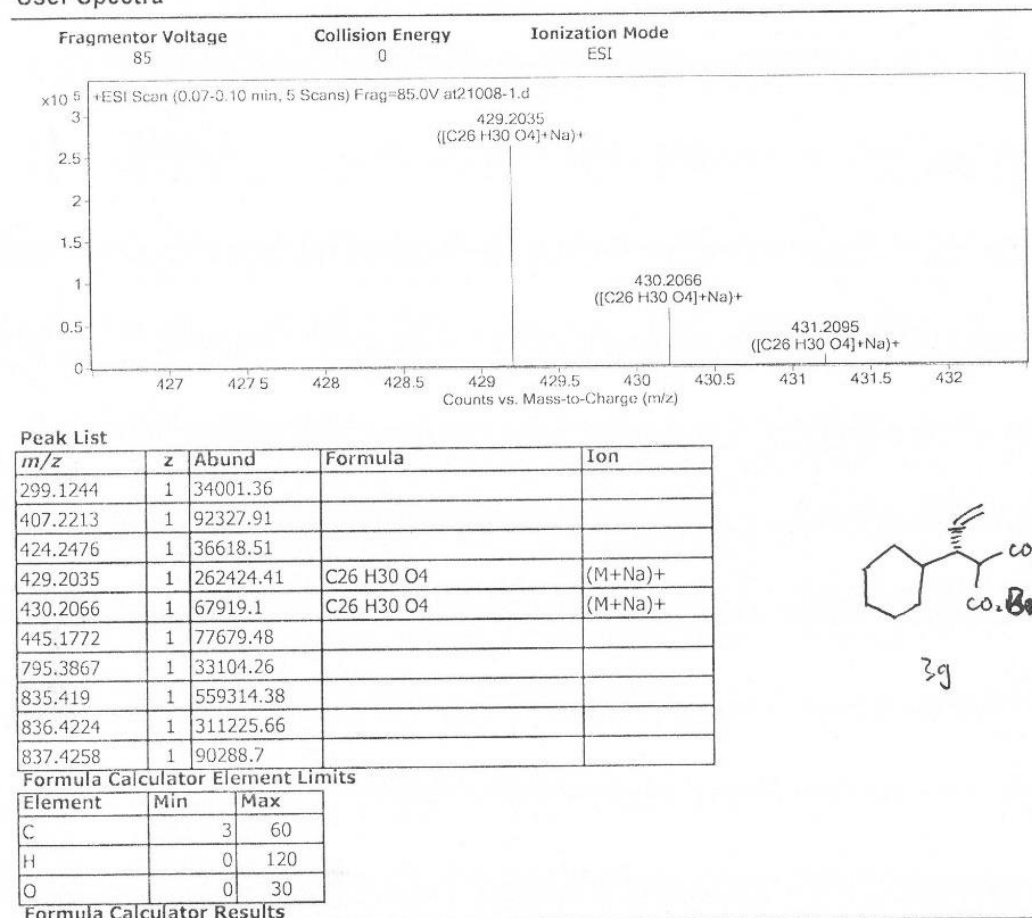

\begin{tabular}{|l|r|r|r|r|r|r|}
\hline Formula Calculator Results & CalculatedMass & CalculatedMz & Mz & Diff. (mDa) & Diff. (ppm) & DBE \\
\hline $\mathrm{C} 26 \mathrm{H} 3004$ & 406.2144 & 429.2036 & 429.2035 & 0.10 & 0.23 & 12.0000 \\
\hline
\end{tabular}

.... End Of Report -.. 
Qualitative Analysis Report

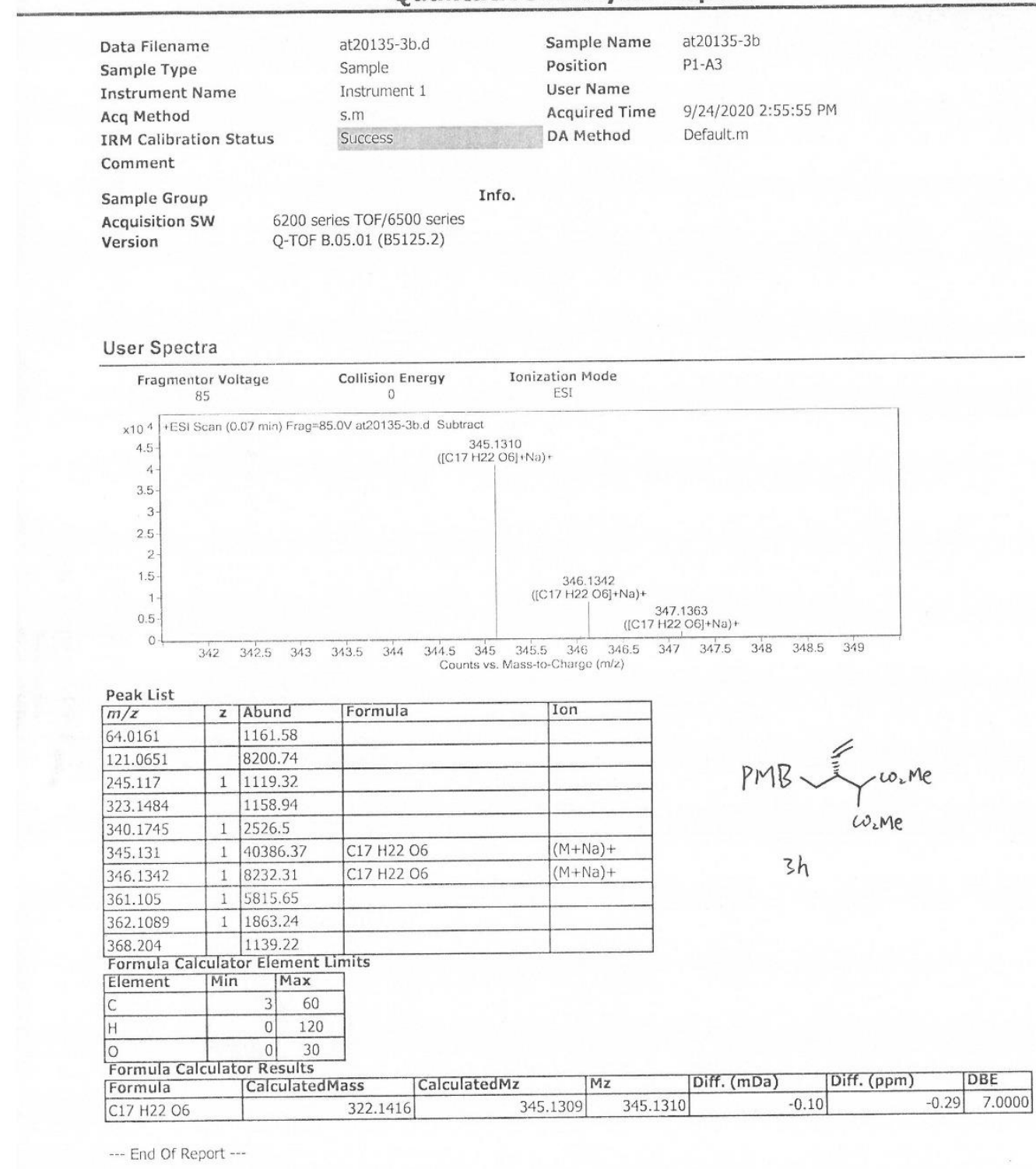


Qualitative Analysis Report

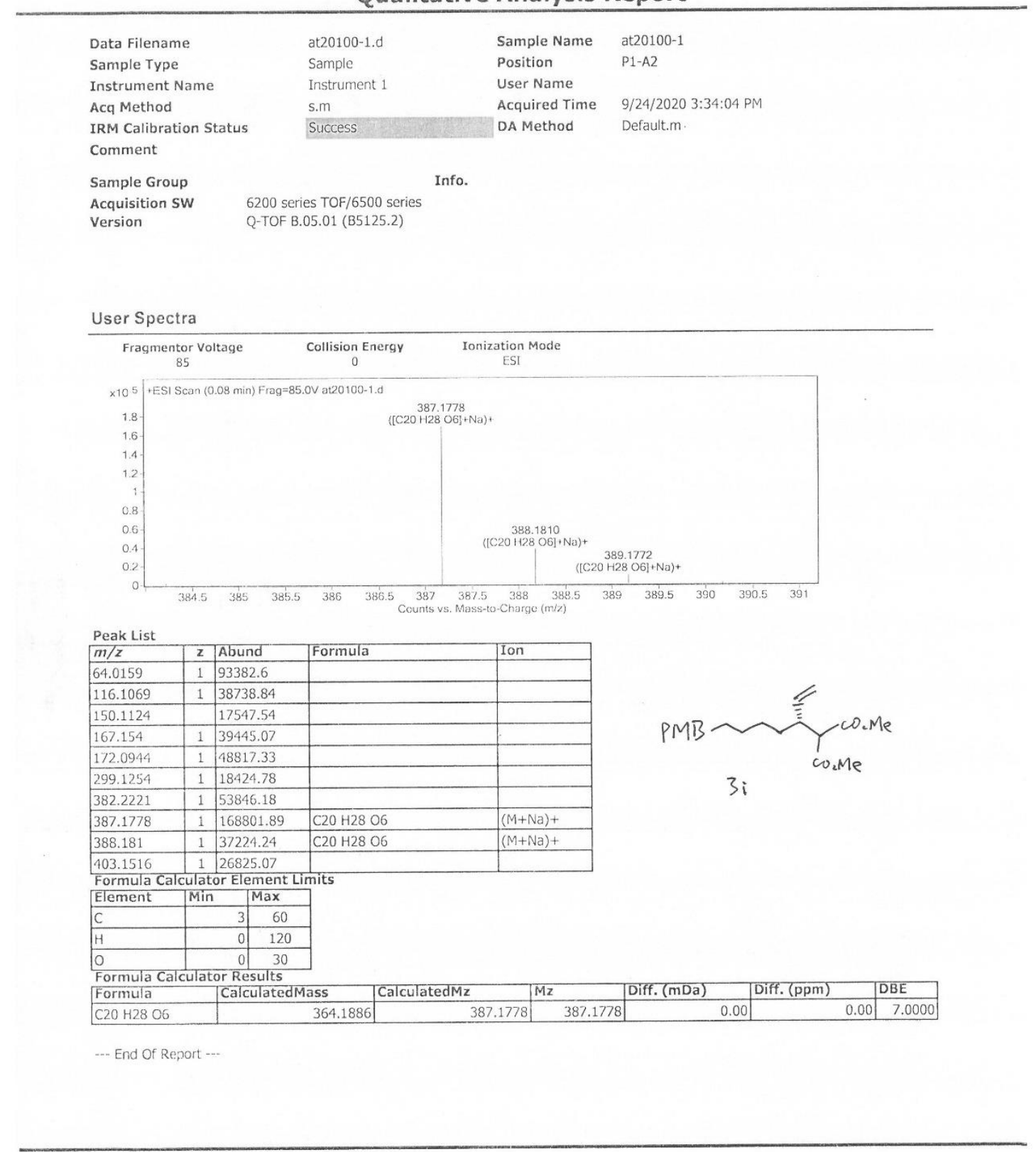

Agilent Technologies

Page 1 of 1

Printed at: $3: 40$ PM on: 9/24/2020 
Qualitative Analysis Report

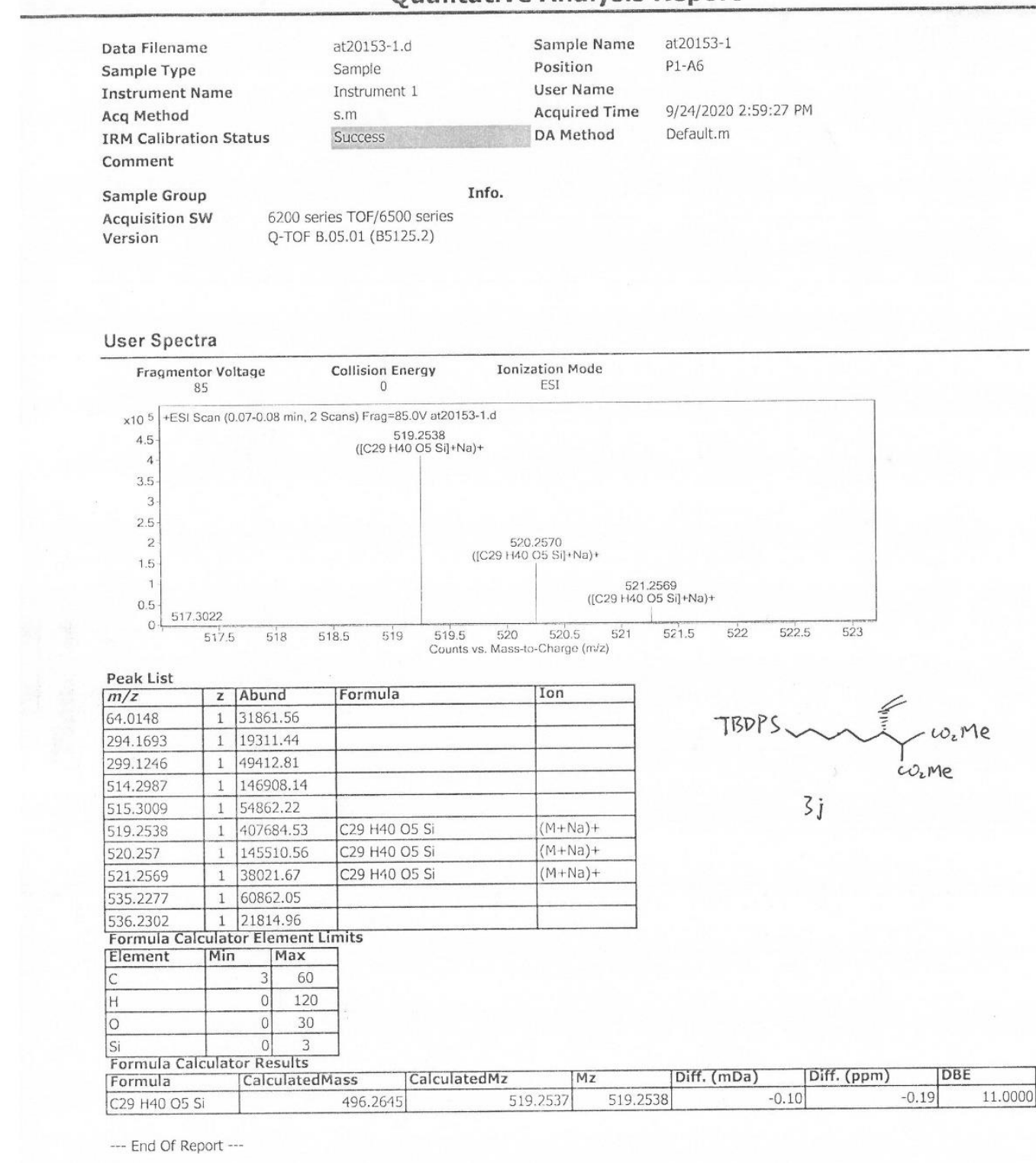

